\title{
POUR RÉÉCRIRE
}

LA FIN DU NÉOLITHIQUE

DANS LE SUD-EST DE LA FRANCE:

\section{BILAN CRITIQUE} SUR CENT ANNÉES D’ÉTUDES

\author{
Jessie CAULIEZ*
}

\begin{abstract}
Mots-clés. Néolithique final, Méditerranée nord-occidentale, Provence, canevas chronoculturel, lecture historiographique, céramique. Résumé. Dans l'arc méditerranéen français, cela fait maintenant plus d'un siècle que les chercheurs en Préhistoire récente portent toute leur attention sur l'élaboration du cadre chronoculturel, ouvrage pour lequel les formes céramiques et les styles décoratifs ont été de première importance pour rendre compte de l'existence d'entités culturelles d'extension géographique circonscrite développant des traits originaux. Cet article propose une remise à plat de toute la documentation régionale et une lecture historiographique de la fin du Néolithique, dans le but de décortiquer les théories en cours sur cette période et leur utilisation dans la construction d'un système explicatif pour lequel, malgré ces honorables années de recherche, tout ou partie des démonstrations repose encore trop souvent sur l'accumulation d'hypothèses empiriques échafaudées sans réactualisation. L'histoire de la construction du canevas chronoculturel du sud-est de la France est ainsi retracée de façon critique en trois grandes parties pour dégager les fondements valides et les incertitudes sur les questions d'origine, d'évolution, de transformation et d'imbrication des groupes est-rhodaniens.

La démonstration montre notamment la primauté languedocienne dans les schémas de formation des groupes provençaux et donc les liens plus ou moins forts dans les relations envisagées entre les cultures de part et d'autre du Rhône. En explorant la dimension géographique donnée à ces groupes et celle de la pertinence de l'échelle à laquelle ils sont définis, la lumière est faite aussi sur la difficulté à ordonner les cultures de manière à rendre compte de leur renouvellement et de leurs différentes variations spatiales (faciès) et chronologiques (possibilité de phasage interne). Enfin, la réintégration des données sur la fin du Néolithique pour un secteur allant de l'Italie centrale au Languedoc oriental et de la côte méditerranéenne jusqu'aux lacs de Suisse occidentale est l'occasion de présenter et de coordonner des informations de sphères géoculturelles variées pour se préoccuper de la réinscription du Néolithique final de Provence dans un cadre beaucoup plus global. L'article s'achève alors sur les premières orientations que nous proposons de suivre dans la résolution des problèmes mis en évidence tout au long de cette lecture croisée.
\end{abstract}

Keys-words. Final Neolithic, north-western Mediterranean, Provence, chrono-cultural framework, historiographic analysis, pottery. Abstract. In the French Mediterranean arch, over more than the past century, specialists in recent Prehistory have been focusing on developing a chrono-cultural framework. In this context, pottery forms and decorative styles have been of primary importance for identifying cultural entities of limited geographic extension, and which developed original traits.

This article presents a critical review of all the French region-wide literature and a historiographic analysis of the end of the Neolithic. The objective is to dissect the current theories on this period and their use in the development of an explanatory system whose demonstrations, despite all these years of research, are still too often based on an accumulation of non-updated empirical hypotheses. Three broad aspects of the history of the chrono-cultural framework development in south-eastern France are thus critically reviewed in order to distinguish valid foundations and remaining uncertainties on the origin, evolution, transformation and imbrications of Eastern Rhone groups.

* Post-doctorante de la fondation Fyssen, LAMPEA, UMR 6636 du CNRS, Maison méditerranéenne des sciences de l'homme, 5, rue du Châteaude-l'Horloge, BP 647, F-13094 Aix-en-Provence Cedex 2. Courriel: cauliez@mmsh.univ-aix.fr 
We show, for example, the primacy of the Languedoc element in the formation of Provençal groups and therefore the more or less strong links in the supposed relationships between cultures on both sides of the Rhône. By exploring the geographic dimension attributed to these groups and the adequacy of the scale at which they are defined, the difficulties in ordering these cultures to reflect their renewals and different spatial (facies) and chronological (possibility of internal phasing) variations also become evident. Finally, the reintegration of data concerning the end of the Neolithic in a zone extending from central Italy to the eastern Languedoc and from the Mediterranean coast to the western Swiss lakes, presents an opportunity to coordinate information from various geo-cultural spheres in order to replace the Final Neolithic of Provence within a much broader context. This paper thus ends with the first research orientations that we propose to follow in order to resolve the problems exposed throughout this integrated analysis.

Translation: Magen O'FARRELL

Voilà maintenant plus d'un siècle que dans le midi de la France, les recherches en Préhistoire récente s'attachent tout particulièrement à dresser les grandes lignes du dernier millénaire avant l'âge du Bronze. Pour la Provence, pendant le Néolithique final, c'est-à-dire entre 3400 et 2400 av. J.-C., une série de groupes culturels a été définie sur la base des formes céramiques et des styles décoratifs, pour rendre compte de l'existence d'entités sociales d'extension géographique circonscrite et ayant développé des traits originaux. Ce compartimentage en groupes culturels, limités dans l'espace et dans le temps, cache cependant mal les incertitudes liées à des documents céramiques peu nombreux, qu'il n'est pas facile d'insérer dans un système explicatif. À détailler la fin du Néolithique en région provençale essentiellement à partir de quelques mobiliers, cette période s'est retrouvée, au fil des années, réduite dans des schémas contraints fondés sur quatre ensembles culturels, Fraischamps, Couronnien, Rhône-Ouvèze et Nord-Vaucluse, progressant de façon linéaire et immuable, dans une périodisation déclinable en deux grands temps et dans un espace inégalement exploité. Dans ces conditions, étudier les mécanismes des évolutions stylistiques de la céramique, un des meilleurs marqueurs que les néolithiciens ont à leur disposition pour décrire les dynamiques culturelles, doit passer par un changement d'attitude par rapport au sujet. Il est en effet nécessaire de traiter spécialement de grandes séries d'artefacts bien calées dans le temps et de provenance stratigraphique fiable, avant une reconstruction dans le cadre plus large du sud-est de la France, pendant ce millénaire où l'on voit une indiscutable densification du peuplement accompagnée par de profondes modifications des fonctionnements sociaux et de la compétition entre les hommes (Cauliez, 2009).

Comment peut-on atteindre cet objectif, pour proposer une interprétation différente du Néolithique final provençal, sans consentir d'abord à faire pas à pas et en sens inverse le chemin de l'histoire de la recherche ? C'est là tout l'enjeu de cet article. Pour mieux comprendre de quelle manière les théories en cours sur la fin du Néolithique estrhodanien se sont construites et surtout pourquoi la vision qui se dégage actuellement de la Provence pour ce stade de la Préhistoire récente est celle d'une sphère cloisonnée, voire figée, un bilan critique des cent dernières années de recherche s'est d'abord imposé comme une priorité. Cette remise à plat de toute la documentation régionale permet de faire la liste des problèmes, au premier rang desquels se trouvent des lacunes méthodologiques évidentes telles que l'identification d'un groupe en privilégiant l'utilisation de quelques critères céramiques a priori significatifs d'une culture archéologique. Elle met également en avant combien les travaux provençaux, pour l'intervalle de temps compris entre la fin du Chasséen et les débuts de l'âge du Bronze, se sont échafaudés en grande partie par analogie avec les trames chronoculturelles du Néolithique languedocien. Dès lors, les schémas de mise en place et d'évolution des cultures provençales sont lus et décryptés dans un sens unilatéral, de l'ouest vers l'est, comme si le Languedoc était la clef pour la compréhension de tous les phénomènes observés sur la rive gauche du Rhône, là où les groupes ne sont purement et simplement qu'assimilés aux cultures voisines de Ferrières et de Fontbouisse en Languedoc oriental. Les dynamiques indépendantes propres à la Provence sont ainsi minimisées. Plus encore, dans les raisonnements formulés sur la formation des groupes, sur leur évolution et sur les possibles processus d'interaction, il n'y a pas de place accordée à d'autres acteurs extrarégionaux, notamment italiens, rhodaniens et jurassiens, alors que tout invite à penser cette période comme emblématique de réseaux complexes et foisonnants régissant les sociétés. Ces bases déconstruites, cet article permet, au fil de la réflexion, d'esquisser de nouvelles problématiques en termes géographiques vers l'Italie ou le Jura, mais aussi en termes chronologiques, plus particulièrement pour le Néolithique récent et les derniers siècles du III ${ }^{\mathrm{e}}$ millénaire av. J.-C. 


\section{LA FIN DU NÉOLITHIQUE EN PROVENCE. POLYMORPHISME GÉOGRAPHIQUE, CHRONOLOGIQUE ET CULTUREL}

Étape intermédiaire entre le Néolithique moyen et les débuts de l'âge du Bronze, la fin du Néolithique constitue, dans l'évolution des sociétés, un stade essentiel marqué par diverses transformations techniques et sociales. Cette période capitale est aujourd'hui perçue au travers de quelques idées communes, dont on peut d'ores et déjà rappeler les linéaments. Pour des compléments, nous renvoyons le lecteur à plusieurs articles qui proposent d'autres bilans (Courtin, 1974; Gutherz, 1984; Bailloud, 1985; Lichardus, Lichardus-Itten, 1985; Beeching, 1986 et 2002; Pétrequin et al., 1987-1988; Voruz, 1991; Voruz dir., 1995; Gutherz, Jallot, 1995; D’Anna, 1995b et 1999; Vaquer, 1998).

\section{UNE ÉCONOMIE DE PRODUCTION DOMESTIQUE FONDÉE SUR LA RÉCIPROGITÉ ET LA COMPÉTITION}

La fin du Néolithique est entendue comme un temps de mutations, d'accélérations et de ruptures, qui survient après le complexe culturel néolithique moyen chasséen et qui donne naissance aux premières communautés de l'âge du Bronze. Le déploiement et les progrès de l'économie agro-pastorale et les nouvelles conquêtes technologiques, comme l'apparition et le développement de la métallurgie, sont parmi les facteurs explicatifs de ces grands bouleversements (Guilaine, 1994, p. 121-122). Ils auraient permis une certaine prospérité entraînant une augmentation significative de la population dont témoignerait le nombre élevé de 600 sites connus en Provence (D’Anna, 1995a, p. 321). L'hypothèse retenue est celle d'une évolution sur place, à partir du fonds chasséen, conduisant à la naissance de plusieurs groupes concurrents issus de cet essor démographique. La civilisation emblématique du Néolithique moyen, ainsi fragmentée par les luttes pour le contrôle des ressources naturelles et particulièrement des terroirs les plus intéressants pour l'exploitation agricole et l'élevage, aurait alors été relayée par de nombreuses unités géoculturelles locales moins expansionnistes, dont les limites ont pu varier au fil du temps (Voruz et al., 1995, p. 388). En Provence, il s'agit des groupes Fraischamp, Couronnien, Rhône-Ouvèze et Nord-Vaucluse (D'Anna, 1995a).

Dans ce moment d'accélération et de bouleversements, la fin du Néolithique offre aux archéologues l'image d'une mosaique de cultures relativement cloisonnées. Toutefois, s'il existe bien des éléments à valeur culturelle régionale, voire locale, dans lesquels le groupe s'identifie, des dénominateurs communs à l'ensemble des cultures du Midi méditerranéen sont avérés. La Provence de la fin du Néolithique s'intègre ainsi au sein d'une cohésion culturelle touchant les différentes provinces du Sud (Beeching, 1984; Gutherz, 1984; Guilaine et al., 1988; Sauzade, 1990a).

\section{DES DÉNOMINATEURS COMMUNS À L'ENSEMBLE DU MIDI MÉDITERRANÉEN}

Dans un contexte de fortes transformations culturelles, l'économie des sociétés du sud-est de la France au Néolithique final est marquée par une augmentation de la production agricole et une intensification de l'élevage. Si la composition des troupeaux est relativement constante - caprinés, ovins et bovins -, une variabilité apparaît dans leur gestion. La part réservée à la chasse est quant à elle fortement réduite (Helmer, 1979 et 1992; Blaise, 2005 et 2007). Actuellement, le manque d'études carpologiques et le faible développement des travaux interdisciplinaires limitent nos connaissances sur le rôle de l'agriculture dans l'économie des groupes humains. Cependant, les nombreux témoins directs comme les graines, et indirects comme les outils en silex, les meules, les broyeurs, les haches, certaines structures en creux (fosses silos) et la céramique, semblent révéler un accroissement des cultures agricoles et une hausse de la production (Lemercier et al., 2004).

Bien que les phénomènes postdépositionnels et les processus érosifs aient pu biaiser la conservation des gisements des périodes précédentes, l'hypothèse d'une augmentation de la population ou, tout du moins, d'une densification du nombre de sites dans le Midi méditerranéen à la fin du Néolithique peut être avancée. Pour le département du Vaucluse, par exemple, on dénombre environ 70 sites connus pour le Néolithique moyen et plus de 280 pour le Néolithique final, soit un nombre quatre fois plus élevé (Lemercier, Blaise et al., 2004). Ce peuplement s'organise en habitat dispersé, formé par une multitude d'établissements plus ou moins réduits, de types fermes, hameaux ou petites exploitations. Ces habitations, réunies en unités dans de vastes espaces de plaine (les Fabrys à Bonnieux dans le Vaucluse, le Collet-Redon à Martigues dans les Bouches-duRhône) ou de terrasse (le Mourre du Tendre à Courthézon dans le Vaucluse), cohabitent avec d'autres sites installés sur des hauteurs, des éperons barrés (la Fare à Forcalquier dans les Alpes-de-Haute-Provence, les Lauzières à Lourmarin dans le Vaucluse, la Citadelle à Vauvenargues et Miouvin 
à Istres dans les Bouches-du-Rhône) dotés souvent d'un système de retranchement: enceintes ou profonds fossés (Camps-Fabrer, D’Anna, 1989; D’Anna, 1993 et 1999).

Les habitations de la culture Fontbouisse en Languedoc offrent également les meilleurs modèles de cette mutation (Guilaine, 1994, p. 156). Les grottes et les abris ne sont pas mis en marge pour autant. On tire aussi profit des plaines et des vallées ouvertes et de nouveaux points d'ancrage apparaissent en particulier sur des aires jusqu'ici délaissées, les plateaux et les zones submontagnardes, ce qui conduit à une large dispersion des implantations, une plus forte emprise de l'homme sur son milieu et une meilleure adaptation de l'économie agropastorale aux possibilités de l'environnement.

À l'échelle du Midi, la fin du Néolithique est probablement une période de changements idéologiques avec la diffusion du mégalithisme et la pratique de l'inhumation collective, l'apparition d'un art anthropomorphe et d'une monumentalité importante aussi bien pour l'habitat que pour les sépultures. Ces transformations qui s'enracinent clairement dans les périodes antérieures suivent un processus évolutif qui atteint une sorte de paroxysme au III $^{\mathrm{e}}$ millénaire. Au Mont-Bego, dans les Alpes de l'arrière-pays niçois, entre $2000 \mathrm{~m}$ et $2700 \mathrm{~m}$ d'altitude, des milliers de figurations ont été gravées sur les rochers de schiste. Beaucoup sont datées aux environs de 2000 av. J.-C. (Guilaine, 1994). Du Minervois à la Côte d'Azur, l'arc méditerranéen connaît aussi une floraison de sépultures collectives, ce qui témoigne sans doute de changements dans les structures sociales liés à l'essor démographique, tout comme des liens qui unissent les individus. Ces sépultures collectives sont en grotte naturelle, en dolmen, dans des tumulus à chambre ronde et en hypogée (Guilaine, 2000). En Languedoc, les cavités artificielles formées d'une chambre rectangulaire sont nombreuses: la Clausonnette à Sabran dans le Gard ou le Pioch-Redon à Saint-Jean-deVédas dans l'Hérault, pour n'en citer que quelques-unes, remonteraient au moins au début du III $^{\mathrm{e}}$ millénaire. Les dolmens, composés d'une chambre quadrangulaire précédée d'un couloir, se multiplieraient eux aussi à la transition du IV ${ }^{\mathrm{e}}$ au III ${ }^{\mathrm{e}}$ millénaire, comme celui du Lamalou à Rouet dans l'Hérault, un des mieux conservés (Guilaine, 1994, p. 261). En Provence, les grottes sépulcrales se développent durant une première phase du Néolithique final, tandis qu'un second temps voit l'apparition d'hypogées, comme ceux découverts en remontant la vallée du Rhône ou encore ceux de Fontvieille en Basse-Provence, construits dès le début du III ${ }^{\mathrm{e}}$ millénaire (Sauzade, 1990a; D’Anna, 1995b, p. 279). La perception d'une cohérence culturelle repose également sur la présence de statues-menhirs et de stèles anthropomorphes dans la France méridionale «presque sans interruption du Tarn aux Alpes» (Camps, 1989; D’Anna et al., 2004). En Provence, deux groupes stylistiques sont identifiés: les stèles dites à cupules ou de type venaissin (Camps, 1989), que l'on rapproche des stèles languedociennes, et les stèles à chevrons ou duranciennes (Camps, 1989; Jallot, D’Anna, 1990; D’Anna, 1995b). De récentes découvertes ont conduit à définir un nouveau groupe de stèles caractérisé par l'absence de décor sculpté ou gravé, comme les stèles de Goult (Vaucluse) et de Château-Blanc (Ventabren, Bouches-du-Rhône).

La culture matérielle, en même temps qu'elle permet de différencier des entités culturelles, révèle des parentés dans les styles et des homogénéités techno-économiques. Là encore, elle témoigne du fait que les groupes de la fin du Néolithique "participent bien d'un même ensemble et qu'ils constituent différents aspects d'une même société» (D’Anna, 1995b, p. 278). De nombreux parallélismes stylistiques - formes carénées, décors de cannelures, de pastillage, etc. - entre la céramique des groupes du Néolithique final languedocien et provençal en constituent des exemples saillants (Ferrières ou Fontbouisse à l'ouest du couloir rhodanien et Rhône-Ouvèze, Nord-Vaucluse ou Fraischamp à l'est). Sans mésestimer les lacunes dans les connaissances des autres composants de la culture matérielle (Sénépart, 1995; Voruz et al., 1995), l'industrie lithique et osseuse révèle tout autant une communauté d'éléments entre les groupes. Dans l'industrie lithique, l'utilisation d'un silex local est par exemple prépondérante. Les populations du III $^{\mathrm{e}}$ millénaire av. J.-C. tirent au maximum parti des potentialités régionales. Une plus grande exploitation des matériaux autochtones est de mise, après la densification du peuplement qui nécessite une multiplication des sources d'approvisionnement (Guilaine, 1994). Dans l'outillage de pierre, on voit notamment prospérer en Provence les outils laminaires en silex blond des monts du Vaucluse et du mont Ventoux, déjà connus dans la période précédente (Léa, 2004), mais aussi les lames en silex bruns rubanés oligocènes de la vallée du Largue dans les Alpesde-Haute-Provence, et celles en silex barrémo-bédouliens des environs de Sisteron dans le même département et de la Nerthe dans les Bouches-du-Rhône (Renault, 1998 et 2006; Binder et al., 1998; Lombard, 1999). Plus généralement encore, c'est la taille du silex par percussion directe à la pierre qui domine, avec comme but principal la production d'une quantité importante d'éclats destinés à servir de 
supports au façonnage d'outils. Pour cela, des techniques diverses sont utilisées. La retouche à l'aide d'un percuteur en bois (animal ou végétal) ou en pierre et la retouche à la pression sont la plupart du temps mises en œuvre pour réaliser des grattoirs, des perçoirs et des coches. Parallèlement à ce matériel commun, un outillage particulier composé d'armatures et de lames prend une place prépondérante (Furestier, 2005). Dans l'industrie osseuse, la panoplie des outils comprend surtout des instruments pointus et biseautés, débités par fractionnement et façonnés par abrasion. Le spectre des faunes utilisées dans la fabrication de ces ustensiles est semblable dans tous les groupes: les caprinés et les bovinés, dans lesquels sont privilégiés les tibias et les métapodes (Provenzano in Lemercier, Blaise et al., 2004, p. 214).

\section{DES RÉSEAUX D'ÉCHANGE ÉTOFFÉS}

Les relations à plus ou moins longue distance sont perçues à travers des réseaux d'échange étoffés et offrent aux archéologues l'image d'un monde relativement homogène, fondé sur la réciprocité et la concurrence (Beeching, 1986; Guilaine, 2000).

Ces circuits d'échanges peuvent porter sur des matériaux utilitaires: objets finis ou matières premières brutes. L'exemple des haches polies d'origine liguro-piémontaise, essaimées ici et là dans tout le Midi, le suggère fort bien (Thirault, 2004). Des objets socialement valorisés voyagent aussi, comme les poignards en silex du Grand-Pressigny (Touraine, Indre-et-Loire) découverts dans les sites du Sud-Est lors des fouilles préventives réalisées sur le tracé du TGV Méditerranée (Mallet et al., 2000) ou encore celui en cuivre de type Remedello (Cornaggia Castiglioni, 1971) révélé dans le dolmen du Mas des Gavots à Orgon dans les Bouches-du-Rhône (Courtin, Sauzade, 1975; BargeMahieu, 1995). Des pétroglyphes représentant fidèlement ces poignards caractéristiques de l'Eneolitico d'Italie septentrionale sont également présents sur des rochers aux abords du passage qui relie Cuneo et la vallée de la Durance aux Oullas dans les Alpes-de-Haute-Provence (Courtin, Sauzade, 1975; Rossi, Gattiglia, 2005; Morin et al., 2005 et 2006). Enfin, l'essentiel de la métallurgie chalcolithique du cuivre identifiée dans le midi de la France, y compris celle reconnue en Provence, quoique très secondaire, est d'origine fontbuxienne (Ambert, 1998). Le groupe languedocien Fontbouisse, qui est connu pour l'exploitation intensive de ressources cuprifères du sud du Massif central dès le début du III ${ }^{\mathrm{e}}$ millénaire (Ambert et al., 2005), voit ses productions métalliques se propager jusqu'en Suisse occidentale, propagation qui témoigne de cette dynamique (Strahm, 2005, p. 32).

La sphère méridionale n'est pas seulement un lieu réceptif aux échanges. Des circuits à large échelle semblent aussi établis depuis des jalons régionaux. Les influences provençales sont connues par l'exportation de lames en silex lacustre oligocène de la région de Forcalquier ou en silex bédoulien du mont Ventoux (Vaquer, 2007). Ces produits laminaires circulent à partir d'ateliers spécialisés, sur de grandes distances, sous la forme de produits finis ou semi-finis, puisqu'on les signale en Italie du Nord, en Suisse occidentale, dans la zone nord-pyrénéenne et en Espagne (Renault, 1998; Honegger, 2001 et 2006, p. 46; Vaquer, 2007, p. 71). On sait depuis peu que ces silex transitent aussi sous la forme de productions laminaires jusque dans le Valais au début du III ${ }^{\mathrm{e}}$ millénaire - dolmen de Sion MXII (Honegger, 2001) -, en Suisse occidentale dans le niveau 1C de Portalban (ibid., p. 211) et dans le Jura à Clairvaux, - la Motte-aux-Magnins, station IV et Chalain, stations IV et XIX - dès la fin du IVe millénaire (Honegger, 2006). L'existence des poignards en silex de Forcalquier procéderait d'un mécanisme complexe visant à reproduire des exemplaires métalliques exogènes, en particulier italiens, ou à rivaliser avec ces derniers, tout du moins sur le plan symbolique (Bailly, 2002; Honegger, 2006, p. 48; Renault, 2006, p. 159; Vaquer et al., 2006). La période qui nous intéresse est aussi celle de la prolifération des perles en stéatite et autres pendentifs en os, coquillage, calcaire et calcite, qui se répandent très largement depuis le Languedoc jusque dans les habitats lacustres du Jura (Barge, 1982; Pétrequin et al., 1987-1988). Enfin, à cette époque où se développent les statues-menhirs et les stèles anthropomorphes, il est par ailleurs admis que les monuments mégalithiques provençaux, comme les dolmens et les hypogées de Fontvieille, constituent des points d'origine d'une architecture singulière qui aurait ensuite rayonné de part et d'autre du Rhône (Jallot, D’Anna, 1990; Sauzade, 1990a, p. 316; Guilaine, 2000).

Parallèlement, la fabrication d'objets de facture exotique matérialise ces échanges. On peut insister longuement sur la constance des influences du Languedoc oriental au cours du Néolithique final. Depuis 3400 av. J.-C., celles-ci sont récurrentes et bien illustrées dans la céramique provençale, dans laquelle de multiples phénomènes sont identifiés : imitation, transfert stylistique ou circulation entre les groupes Ferrières ou Fontbouisse de la rive droite du Rhône et ceux de Fraischamp, Rhône-Ouvèze et Nord-Vaucluse sur la rive gauche (Giligny, 1993 et 1997). Les styles langue- 
dociens céramiques Ferrières et Fontbouisse sont aussi diagnostiqués jusque dans le Velay et le Jura (D’Anna, 1995b; Gutherz, Jallot, 1995). Ce comportement d'emprunt culturel se retrouve sur les récipients en bouteille à col cylindrique et carène médiane connus dans la Drôme et qui sont considérés comme relevant du groupe Rinaldone (Margarit, Saintot, 2002). Cette culture, longtemps non datée par le radiocarbone, débuterait à la charnière du IVe III $^{\mathrm{e}}$ millénaire av. J.-C. pour disparaître vers le milieu du III ${ }^{\mathrm{e}}$ millénaire av. J.-C. (Conti et al., 1997, p. 180 ; Carboni, 2002, p. 285). Elle s'étend sur la façade tyrrhénienne dans le nord du Latium et le sud de la Toscane (Cremonesi, 1998; Miari, 1998).

Par conséquent, on voit que dans le Midi méditerranéen, le Rhône, pas plus que le Massif central ou les Alpes, ne constitue une frontière infranchissable.

\section{UN MODÈLE DE SOCIÉTÉS}

Toutes ces activités (production, consommation et échange) s'inscrivent dans un type de fonctionnement que A. Gallay a modélisé pour les sociétés néolithiques (Gallay, 1995). Celui-ci peut se résumer de la façon suivante.

Ces sociétés sont des communautés qui se renouvellent par la tradition et les mécanismes de la parenté, laquelle constitue le cadre structurant de la production et de la circulation des objets socialement valorisés. Les choix techniques et/ou stylistiques dans la culture matérielle peuvent être stables pendant des siècles; ils traduisent la récurrence de conceptions véhiculées par l'apprentissage. Le mode de production et de consommation est villageois et s'insère dans le cadre d'une économie domestique ${ }^{1}$. De petites unités forment des groupes sociaux bien compartimentés et fortement structurés autour de maisonnées, mais aussi de territoires agricoles, d'élevage et de chasse. Le tout est compris dans un finage et circonscrit par des zones frontières. Le maintien de la cohésion au sein de la communauté repose sur l'équilibre entre l'autonomie du groupe et sa capacité à échanger. Il nous faut conce-

1. Le concept de «mode de production domestique» forgé et débattu par les marxistes présente bien des faiblesses qui rendent délicat son usage (Dupuy, 2004, p. 149-157). Ici, «mode de production domestique» n'est pas pris dans le sens de M. Sahlins (Sahlins, 1976). On s'appuie, pour partie seulement, sur la définition qu'en donne C. Meillassoux: le "mode de production lignager» est capable de dégager un surplus économique et n'est donc nullement voué à l'économie de subsistance, comme l'entend M. Sahlins. La communauté lignagère pilote et fédère la production économique et organise la gestion des surplus (Meillassoux, 1977). voir des réseaux régis par le troc et la compétition entre individus, le don et le contre-don (Mauss, 1973; Renfrew, Cherry, 1986; Godelier, 2008). Ceux-ci enclenchent et entretiennent un rapport d'obligations réciproques entre les groupes. Ils matérialisent un lien social, tout en façonnant la différenciation. L'alliance et la compétition sociale apparaissent comme les deux facteurs pour stimuler de tels réseaux. On souhaite acquérir des biens rares, par leur matière ou le temps de travail qu'ils représentent, afin de se distinguer socialement, de marquer son ascendance et son identité (Bourdieu, 1979). Dès lors, les cultures sont perméables aux transferts d'idées, d'objets, de symboles et de techniques. Les contacts se multiplient et les terrains d'approvisionnement s'élargissent. Pour que le groupe parvienne à cet équilibre, une condition est requise, celle d'une force dirigeante capable de «contrebalancer les tendances centrifuges des maisonnées et d'imposer des formes de collaborations temporaires » (Gallay, 1995, p. 277). Cette force est légitimée ou instaurée par la prégnance d'un ancêtre commun, réel ou fictif dans l'identité collective (Godelier, 1982; Lemonnier, 1990). Dans ce cadre et sous cette hiérarchie, l'organisation de la production vivrière, la spécialisation productive et la redistribution des ressources sont garanties. En même temps, des réseaux d'échange avec d'autres groupes existent; ils légitiment l'inégalité socioéconomique et la revendication de l'identité du groupe contre l'altérité des voisins.

\section{DÉFINITION DES GROUPES CULTURELS PROVENCAUX}

L'image d'un système culturel large se retrouvant dans tout le Midi méditerranéen semble peu à peu se dessiner (Voruz et al., 1995, p. 389). Dans le schéma tel qu'il est établi, toutes les régions du sud de la France sont cependant fractionnées en de multiples unités géoculturelles à la fin du Néolithique. Le renouvellement de la population aurait eu pour corollaire direct une inclination permanente des individus et des sociétés à vouloir se distinguer de leurs voisins pour affirmer leur autonomie (Beeching, 1984; Gutherz, 1984; Jallot, D’Anna, 1990) et le besoin pour chacun de marquer son identité. Les éléments de la culture matérielle (céramique, habitat, sépulture, etc.) véhiculeraient alors symboles sociaux, signes de distinction et marqueurs d'appartenance à un groupe (Pétrequin, 2002). Cependant, la mise en évidence de frontières culturelles repose essentiellement, pour ne pas dire exclusivement, sur la distinction des styles céramiques, porteurs d'un investis- 
sement identitaire particulier (Lemonnier, 1986; Pétrequin et al., 1994). C'est ainsi que quatre ensembles, Fraischamp et Couronnien dans une première phase, puis Rhône-Ouvèze et Nord-Vaucluse ensuite, domineraient dans l'orbe provençal du Néolithique final la tranche de temps comprise entre 3400 et 2400 av. J.-C.

\section{LE FRAISCHAMP}

La reconnaissance du groupe de Fraischamp par G. Sauzade à partir de la céramique de l'habitat de la Clairière à La Roque-sur-Pernes dans le Vaucluse est relativement récente (Sauzade et al., 1990). Peu de sites lui sont actuellement attribués: la grotte Saint-Gervais à Bonnieux, la Baume des Enfers, la Grande grotte, ou la grotte du Trou Puceu, toutes trois à Cheval-Blanc et l'abri de Sanguinouse à La Roque-sur-Pernes (fig. 1). L'extension géographique du groupe de Fraischamp est en conséquence mal connue; il est pour le moment situé dans les extrémités occidentales du Luberon et des monts du Vaucluse et probablement dans la plaine du Rhône, aux alentours de la confluence avec la Durance (D’Anna, 1995b).

La détermination des attributs céramiques, lithiques et des parures de ce groupe stylistique s'appuie sur les jalons presque uniques de la série de référence de la Clairière; les cinq autres établissements, dont l'implantation est systématiquement en grotte ou en abri, ont été fouillés anciennement et ne proposent finalement pas de données exploitables. Aucun travail de recherche comparable à ceux entrepris pour les ensembles culturels voisins n'est venu enrichir sa définition depuis son invention. La synthèse proposée il y a maintenant plus de dix ans par A. D'Anna reste donc toujours d'actualité (D'Anna, 1995a). C'est à partir du site éponyme que les quelques caractéristiques du groupe sont aujourd'hui listées.

Le style céramique du Fraischamp s'illustre le plus nettement dans ses décors. Les ornementations présentent une grande variété technique qui comprend des gravures, des incisions à la pointe mousse ou aiguë et des impressions pour les décors en creux. Les motifs sont très panachés et le décor en chevron domine l'ensemble. Plusieurs agréments plastiques s'y ajoutent, quelquefois combinés à un décor en creux. Ils se limitent à des cordons lisses, rectilignes, ondés ou en arceaux, uniques ou multiples reliant souvent les préhensions et des cordons courts verticaux.

Comme il est courant dans les groupes du Néolithique final en Provence, l'industrie lithique se compose de lames, de flèches tranchantes, de flèches foliacées symétriques ou non, de flèches losangiques, de grattoirs réguliers sur éclats, de racloirs sur éclats, de becs ou de pointes.

L'industrie osseuse est également peu discriminante; elle rassemble des poinçons sur métapodes fendus longitudinalement, des poinçons sur tibias aménagés en biseau, des pointes sur fragments de diaphyse, des bipointes et des outils à tranchants distaux sur métapodes ou cubitus de bœuf. Elle fait principalement appel à l'aménagement par abrasion et affûtage et au débitage longitudinal, soit par sciage, soit par percussion. La parure présente quelques caractères originaux: éléments en coquillages (cardium, dentale, cérithe, perles discoïdes en test), canines de canidés et pendeloques coniques à pointe calcaire (D'Anna, 1999, p. 149). Quant à l'habitat ou aux sépultures, les connaissances sont pratiquement inexistantes. Enfin, on rapporte au groupe de Fraischamp, les stèles dites à cupules ou de type venaissin, proches des stèles languedociennes (Camps, 1989; Jallot, D’Anna, 1990; D’Anna, 1995b; D’Anna et al., 2004).

\section{LE COURONNIEN}

Le Couronnien est le plus anciennement défini, en 1947, par M. Escalon de Fonton à la suite des fouilles du site éponyme du Collet-Redon à Martigues dans les Bouchesdu-Rhône (Escalon de Fonton, 1947, 1954, 1956, 1963 et 1964). C'est aussi le groupe le mieux connu et le plus documenté (fig. 2). Impulsées par M. Escalon de Fonton, les recherches sur le Couronnien ont été reprises sur plusieurs décennies, d'abord par J. Courtin (1974), puis par A. D'Anna et l'équipe du Projet collectif de recherche sur le Couronnien (Lemercier et al., 2007). Depuis 1999, le site éponyme du Collet-Redon est également réinvesti en vue d'acquérir de nouvelles données sur les diverses phases d'occupation qui se sont succédé depuis les premières installations Néolithique final couronniennes jusqu'à l'âge du Bronze (Durrenmath et al., 2003 et 2007; Cauliez et al., 2007). Même si l'extension du groupe reste à préciser à l'intérieur des terres, il est intégré à la Basse-Provence occidentale et s'étend depuis la côte au sud jusqu'au bassin d'Apt au nord; d'ouest en est, on le retrouve des Alpilles jusqu'au massif des Maures (D’Anna, 1995b).

Là encore, le site éponyme et quelques autres établissements, comme les Lauzières à Lourmarin, la Brémonde à Buoux tous les deux dans le Vaucluse, la Citadelle à Vauvenargues, Miouvin à Istres, ou Ponteau-Gare à Martigues tous les trois dans les Bouches-du-Rhône (D’Anna, 1991, 1993 et 1995a), demeurent les seuls référents 


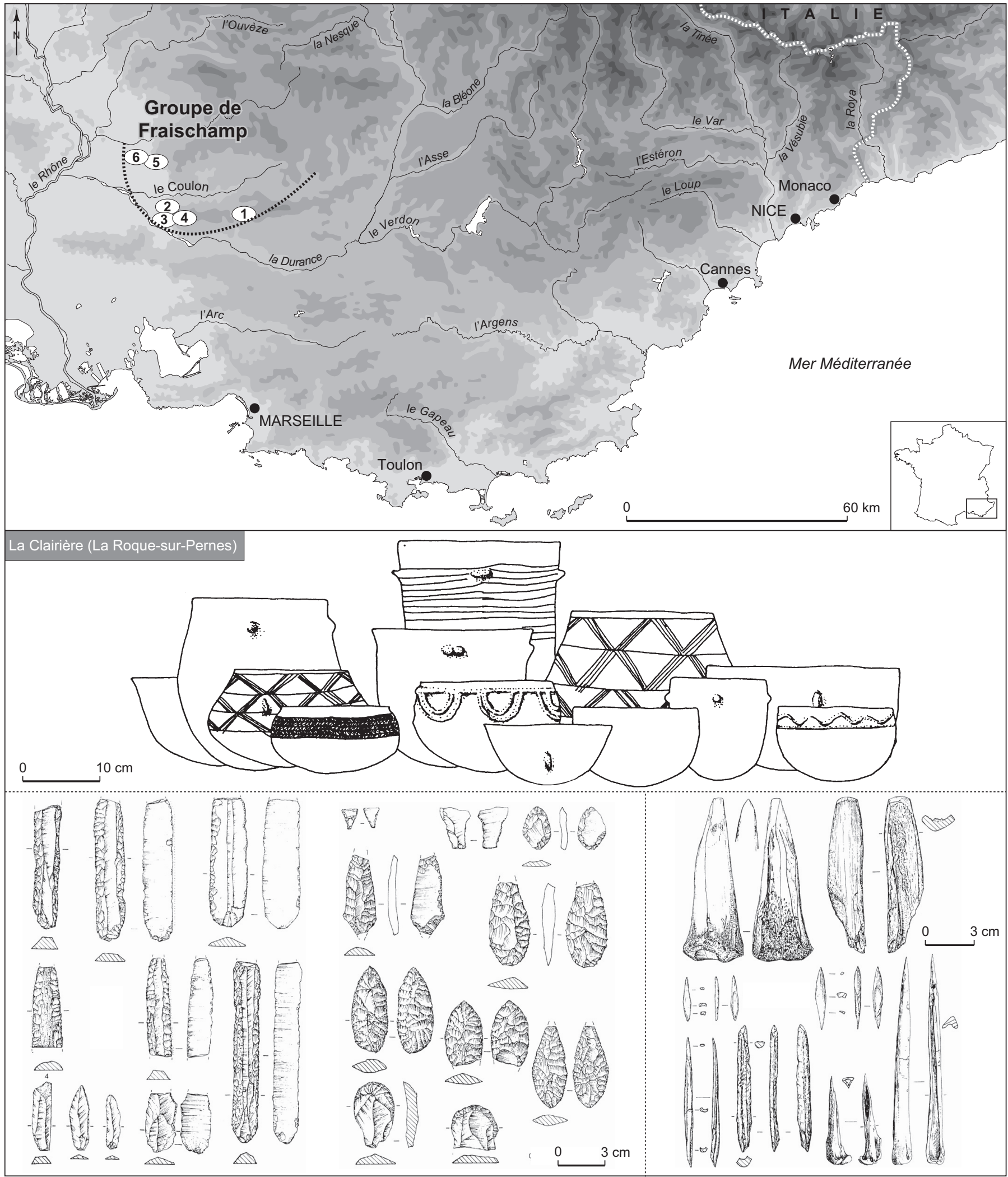

Fig. 1 - Localisation du groupe de Fraischamp dans le Vaucluse: 1, grotte Saint-Gervais (Bonnieux); 2, Baume des Enfers (Cheval-Blanc); 3, Grande grotte (Cheval-Blanc); 4, grotte du Trou Puceu (Cheval-Blanc); 5, la Clairière (La Roque-sur-Pernes); 6, abri de Sanguinouse (La Roque-sur-Pernes). Céramique et industrie lithique et osseuse de l'habitat de la Clairière (La Roque-sur-Pernes) (DAO: J. Cauliez; céramique d'après D’Anna, 1999; industrie lithique et osseuse d'après Sauzade et al., 1990). 


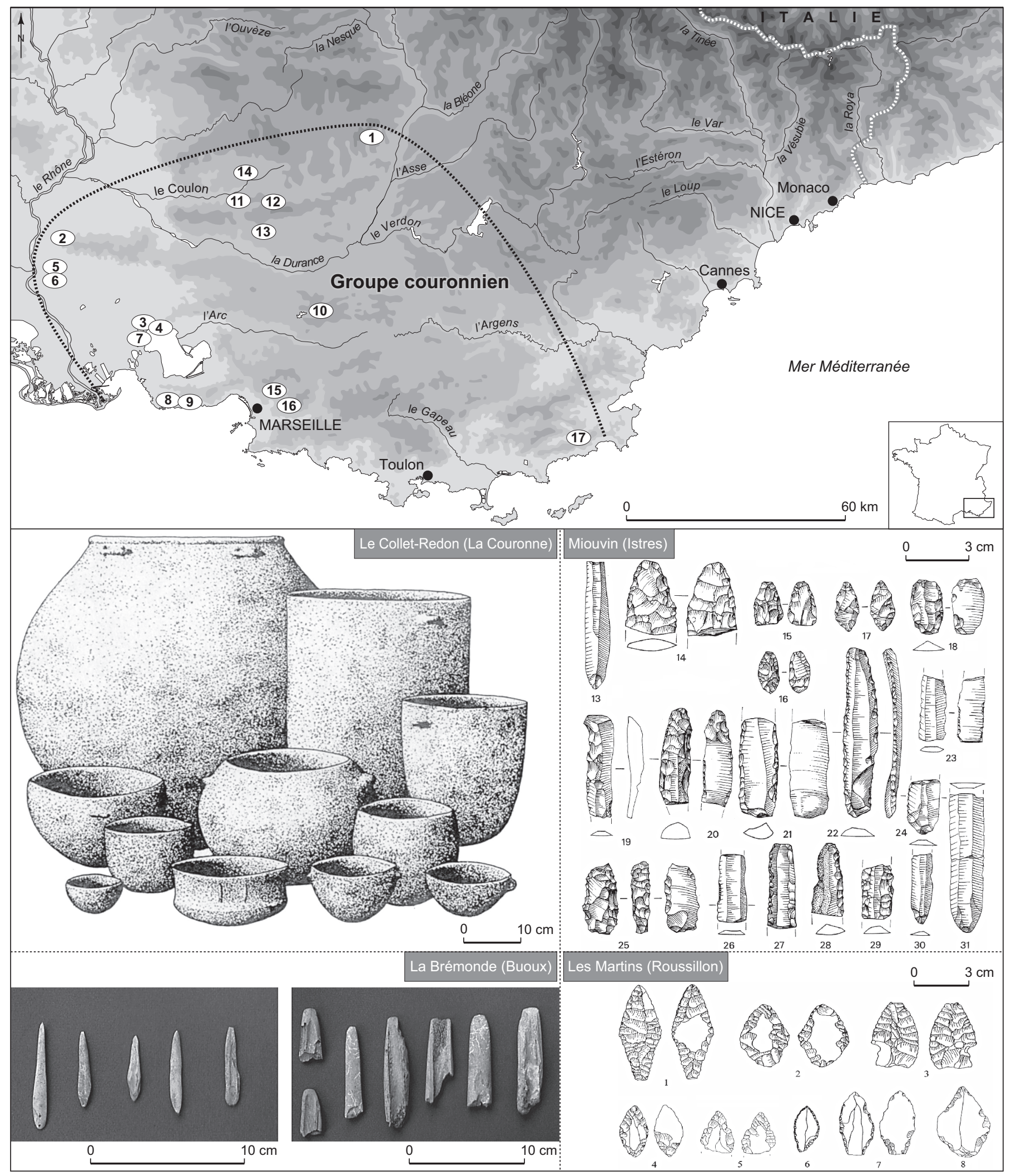

Fig. 2 - Localisation du groupe couronnien: 1, la Fare (Forcalquier, Alpes-de-Haute-Provence); 2, Escanin I et II (Baux-de-Provence, Bouches-du-Rhône); 3, la Bastide Leydet (Cornillon-Confoux, Bouches-du-Rhône); 4, le Deven de Barzac (Cornillon-Confoux, Bouches-duRhône); 5, station de la Calade (Fontvieille, Bouches-du-Rhône); 6, station du Castellet (Fontvieille, Bouches-du-Rhône); 7, Miouvin (Istres, Bouches-du-Rhône); 8, le Collet-Redon (Martigues, Bouches-du-Rhône); 9, Ponteau-Gare (Martigues, Bouches-du-Rhône); 10, la Citadelle (Vauvenargues, Bouches-du-Rhône); 11, les Fabrys (Bonnieux, Vaucluse); 12, la Brémonde (Buoux, Vaucluse); 13, les Lauzières (Lourmarin, Vaucluse); 14, les Martins (Roussillon, Vaucluse); 15, Saint-Joseph-Fontainieu (Marseille, Bouches-du-Rhône); 16, le Pilon du Roy (Allauch, Bouches-du-Rhône); 17, Maravielle (La Môle, Var). Céramique et industrie lithique et osseuse des sites du Collet-Redon, de Miouvin, de la Brémonde et des Martins (DAO: J. Cauliez; céramique d'après Escalon de Fonton, 1968; industrie lithique d'après Renault, 1990; dessins d'après de Butler, in Furestier, 2002. 
sur lesquels la définition du Couronnien peut être fondée. Si plus d'une trentaine de sites couronniens sont actuellement recensés, beaucoup n'ont pas fait l'objet de fouilles extensives aussi importantes que celles du Collet-Redon et la plupart sont partiellement publiés. La synthèse globale est donc loin d'être achevée.

Dans la céramique, le répertoire des formes et décoratif se caractérise principalement par une certaine monotonie. Les morphologies sont simples et les ornementations, très ponctuelles, sont généralement des cordons courts horizontaux, verticaux ou obliques.

Pour l'industrie lithique, le débitage est orienté vers une catégorie principale de support: les éclats. La production de grandes lames et de lamelles est également avérée, auxquelles il faut adjoindre des pointes de flèches foliacées bifaces ou à face plane (Furestier, 2002). Quant à l'industrie osseuse, elle propose les mêmes particularités que celle du Fraischamp.

La parure couronnienne a longtemps été décrite comme essentiellement composée de pendeloques en coquillages bruts perforés ou en test de patella, cardium, littorina, murex et columbella. Aujourd'hui, les études montrent une plus grande variété de mobilier, parfois très travaillé, avec de petites perles et des pendeloques en os sur diaphyse et canine de canidé (chien, renard), en roches diverses et en quartz hyalin (Lemercier et al., 2007).

Les sites du Collet-Redon, de Ponteau-Gare, des Lauzières, de la Brémonde, de Miouvin ou de la Citadelle ont révélé plusieurs structures d'habitat qui permettent d'ébaucher les lignes maîtresses de l'architecture couronnienne. Une grande diversité, tant dans la gestion et la mise en place de l'espace habité que dans la réalisation technique des éléments architecturés, est décelable dans les vestiges. D'abord, les techniques de construction allient des matériaux mixtes comme le bois, la terre à bâtir et la pierre. Ainsi, les murs en pierres semblent systématiquement servir d'éléments porteurs ou, au moins, d'appui à une partie de l'architecture en terre. Ensuite, l'appareil et la mise en œuvre peuvent varier: il s'agit de murs à double parement à remplissage interne ou de murs à parement simple. Enfin, les formes et les implantations de l'habitat sont tournées autour d'unités d'habitation mitoyenne regroupées ou non à proximité d'un grand mur pouvant être commun à plusieurs maisonnées. La partie logement est souvent complétée par des aires d'activité et une enceinte ou un enclos (Gilabert, 2002). Les sites sont en plaine, en terrasse ou perchés et ceinturés, installés sur des plateaux d'accès difficile (D’Anna et al., 1989; D’Anna, 1991 et 1993). Dans le domaine sépulcral, il a été proposé, à titre d'hypothèse, de mettre en relation avec le Couronnien les sépultures collectives en cavités ou en monuments mégalithiques de Provence occidentale, comme les dolmens à chambre allongée et les hypogées de Fontvieille (Sauzade, 1990a; D’Anna, 1999, p. 154).

Dans la seconde moitié du Néolithique final, le Couronnien perdure, en conservant une zone de distribution identique, tandis que le groupe de Fraischamp est remplacé par les groupes Rhône-Ouvèze et Nord-Vaucluse (D’Anna, 1995b).

\section{LE RHÔNE-OUVÈZE}

Le groupe Rhône-Ouvèze a été défini, il y a maintenant plus de vingt ans, d'après la série céramique recueillie sur le site de la Plaine des Blancs à Courthézon dans le Vaucluse (Müller et al., 1986). Des similitudes dans les comparaisons avec d'autres assemblages céramiques, tous vauclusiens, tels que la butte Saint-Laurent et le Mourre du Tendre sur la même commune ou encore Claparouse à Lagnes, ont permis de mettre en évidence un cortège de sites analogues et de proposer ainsi la création d'un nouveau groupe culturel (D'Anna, 1995b).

La répartition géographique de ce dernier reprend, en l'étendant, celle du groupe de Fraischamp (fig. 3). Centrée sur la Provence rhodanienne, elle est limitée, au sud par les Alpilles et à l'est par les extrémités du Luberon et des monts du Vaucluse. Pour cet ensemble, une extension vers l'est (vallée de la Durance) est également possible. Vers le nord, ses frontières restent à préciser, notamment par rapport au groupe Nord-Vaucluse. Plus d'une vingtaine de sites sont actuellement rattachés à cet ensemble culturel.

Les formes céramiques sont de profil simple et offrent un registre très diversifié autour de volumes géométriques dérivés de la sphère, du cône ou du cylindre. Parallèlement, le catalogue morphologique est agrémenté de plusieurs formes à carène vive, basse ou médiane. À l'image de la disparité des morphologies, les décors céramiques sont fréquents et divers, tant par leurs techniques de réalisation que par les motifs représentés. Les décors en relief, souvent nombreux, correspondent à des cordons continus rectilignes, en arceaux ou disposés en chevrons simples ou doubles reliant parfois des éléments de préhension. On rencontre aussi du pastillage au repoussé, des cordons courts et des ornementations de petits boutons multiples en ligne. Les décors en creux, plutôt rares, sont de fines cannelures (incisées à la pointe mousse), organisées en métopes, en 


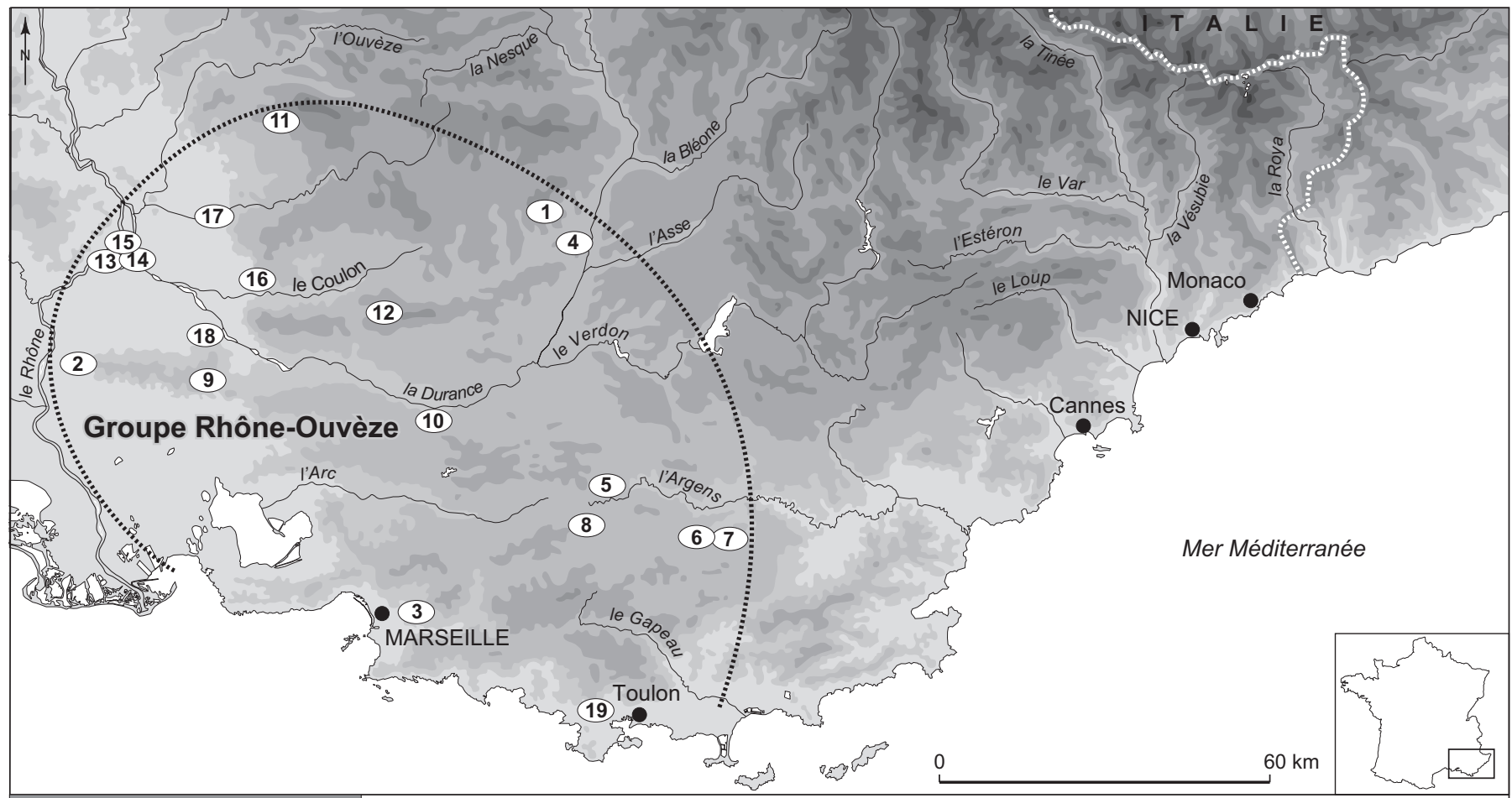

La Plaine des Blancs (Courthézon)
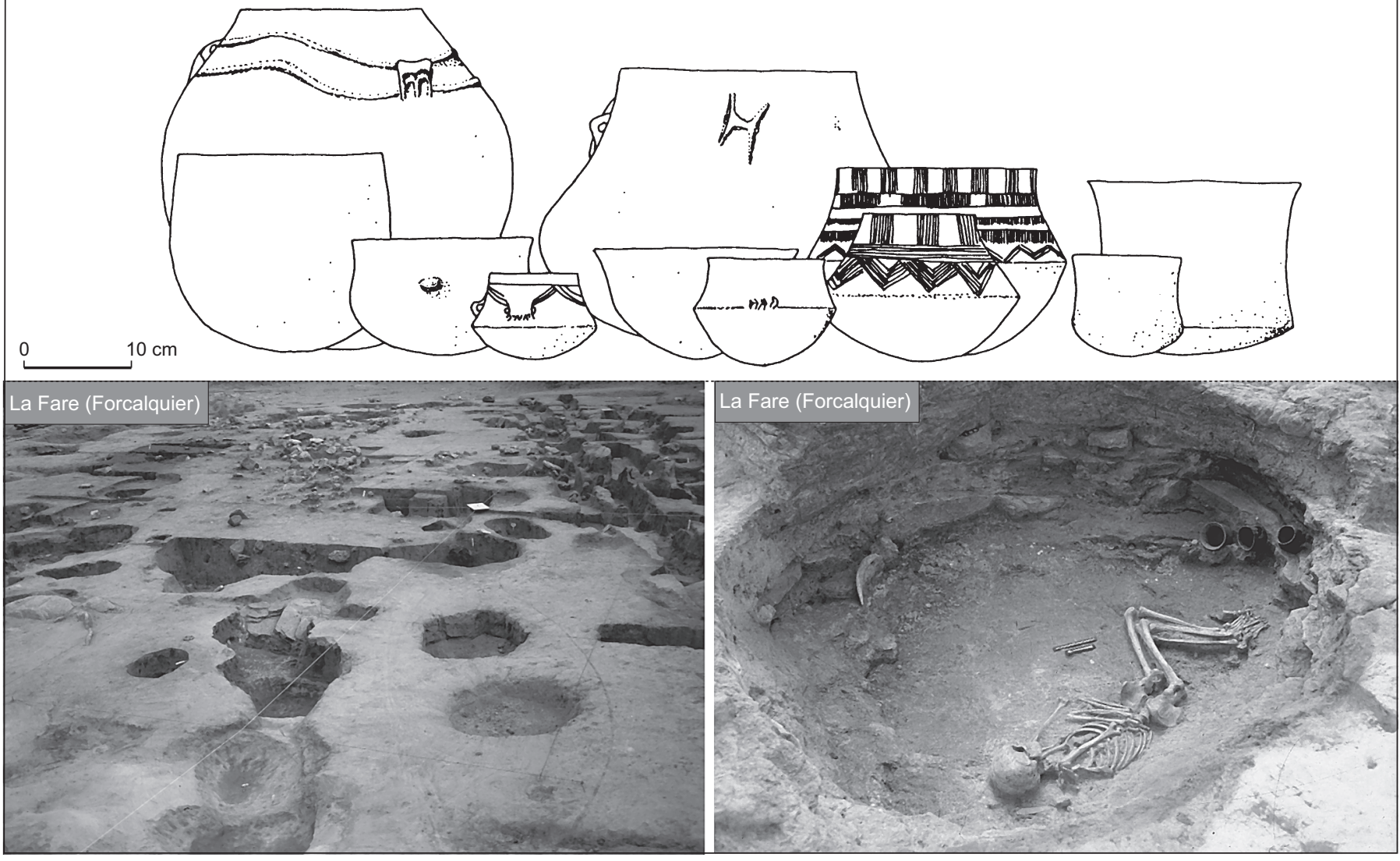

Fig. 3 - Localisation du groupe Rhône-Ouvèze: 1, la Fare (Forcalquier, Alpes-de-Haute-Provence); 2, Escanin I et II (Baux-de-Provence, Bouches-du-Rhône); 3, le Pilon du Roy (Allauch, Bouches-du-Rhône); 4, le Champ du Roi (La Brillanne, Alpes-de-Haute-Provence); 5, la Bastide Collombe (Brue-Auriac, Var); 6, Gazoduc-le Val AO 616 (Brignoles, Var); 7, le Plan-Saint-Jean (Brignoles, Var); 8, le chemin d'Aix (Saint-Maximin-la-Sainte-Baume, Var); 9, les Barres (Eyguières, Bouches-du-Rhône); 10, la Bastide blanche (Peyrolles-en-Provence, Bouchesdu-Rhône); 11, abri de la Madeleine (Bédoin, Vaucluse); 12, les Safraniers (Bonnieux, Vaucluse); 13, le Mourre du Tendre (Courthézon, Vaucluse); 14, la Plaine des Blancs (Courthézon, Vaucluse); 15, la Colline Saint-Laurent (Courthézon, Vaucluse); 16, Claparouse (Lagnes, Vaucluse); 17, la Rambaude (Saint-Didier, Vaucluse); 18, les Passadouires (Orgon, Bouches-du-Rhône); 19, station de Sainte-Estève (Évenos, Var). Céramique du site de la Plaine des Blancs et sépultures sur le site de la Fare (DAO: J. Cauliez; céramique in D’Anna, 1999; clichés A. Müller et O. Lemercier). 


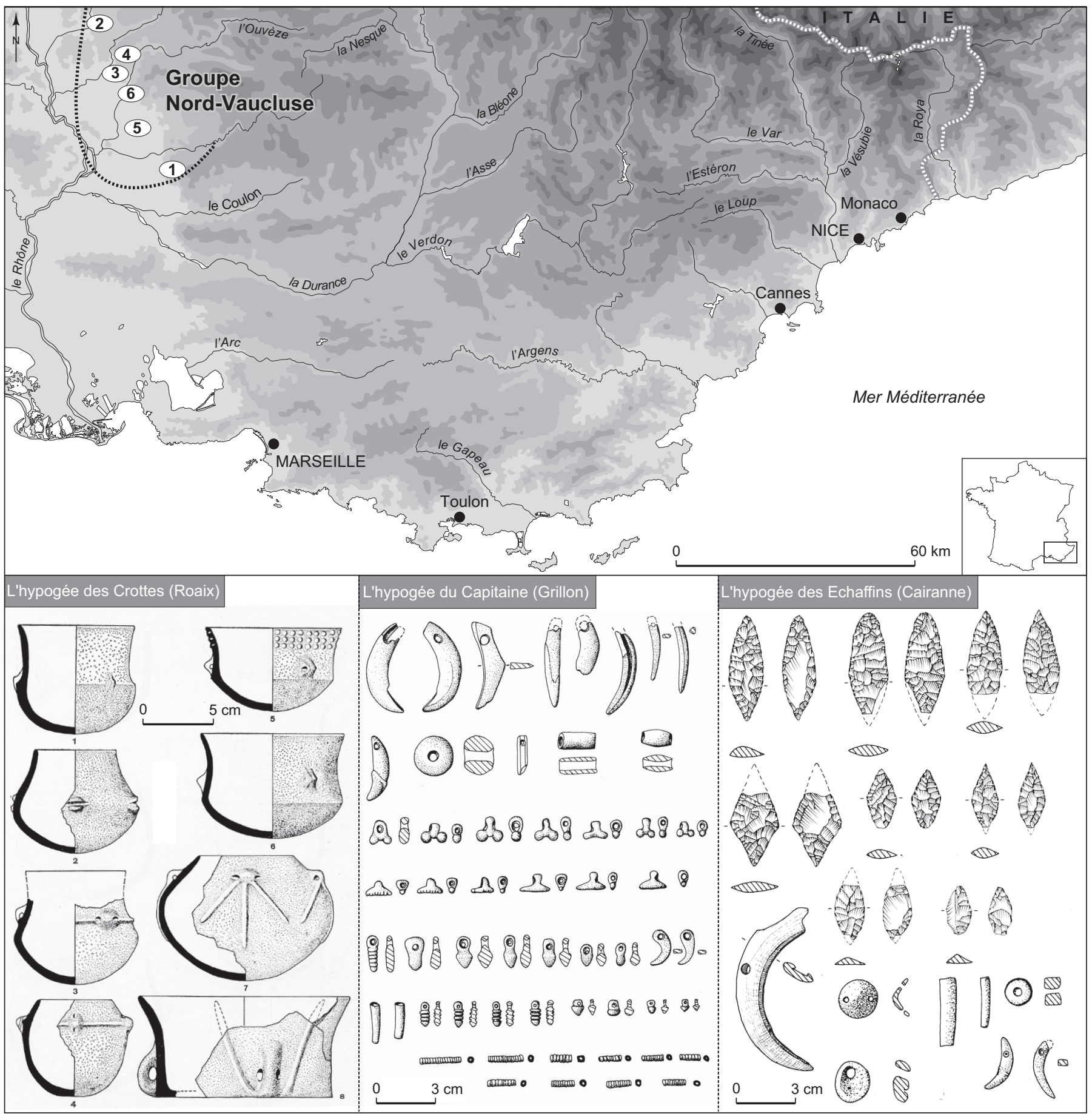

Fig. 4 - Localisation du groupe Nord-Vaucluse: 1, la Clairière (La Roque-sur-Pernes); 2, l'hypogée du Capitaine (Grillon, Vaucluse); 3, l'hypogée des Echaffins (Cairanne, Vaucluse); 4, l'hypogée des Crottes (Roaix, Vaucluse); 5, l'hypogée du Boileau (Sarrians, Vaucluse); 6, les Vignes-Saint-André (Gigondas, Vaucluse). Céramique, industrie lithique et parure des hypogées des Crottes, du Capitaine et des Echaffins (DAO: J. Cauliez; céramique d'après Courtin, 1974; industrie lithique et parure d'après Sauzade, 1983). 
damiers ou en chevrons et souvent associées aux récipients profilés par une carène.

L'industrie lithique n'a pas encore fait l'objet d'études détaillées; elle demeure donc pour le moment difficile à caractériser. De la même manière, l'industrie osseuse reste peu connue. Des données, malheureusement dispersées, peuvent être recueillies dans les travaux de H. CampsFabrer, de D. Helmer ou de S.-Y Choi consacrés à la faune mammalienne et aux outillages en matière dure animale du midi de la France (Camps-Fabrer et al., 1983; Helmer, 1986; Choi, 1999). À l'image du mobilier du Couronnien ou du Fraischamp, ce type d'instrument est réalisé à partir de métapodes ou de tibias de bœufs, de moutons et de chèvres transformés en poinçons, en outils à tranchant distal biface ou aménagés en long biseau.

L'habitat est caractérisé par des aménagements creusés (fosses ou fossés); il est implanté sur des sites de plein air. Les installations sont en pied de pente (Claparouse à Lagnes), sur des terrasses (la Plaine des Blancs et le Mourre du Tendre à Courthézon) ou perchées (la Fare à Forcalquier dans les Alpes-de-Haute-Provence).

À l'exception de la sépulture individuelle en fosse découverte sur le site de la Fare à Forcalquier dans les Alpesde-Haute-Provence, dans laquelle un vase campaniforme et deux vases Rhône-Ouvèze étaient associés (Lemercier, Müller, 1999), les données sur les sépultures du groupe restent lacunaires. Enfin, les stèles à chevrons ou duranciennes pourraient être associées à ce groupe (Camps, 1989; Jallot, D’Anna, 1990; D’Anna, 1995b).

\section{LE NORD-VAUCLUSE}

Au moment de leurs découvertes, des hypogées du nord du Vaucluse ont livré des séries céramiques particulières qui ne se rattachaient directement à aucune des cultures déjà définies (fig. 4). À la frontière septentrionale du Rhône-Ouvèze, G. Sauzade a identifié, au début des années 1990, l'existence d'un quatrième groupe, le Nord-Vaucluse, à partir de trois ensembles funéraires: les hypogées des Crottes à Roaix, des Echaffins à Cairanne ou du Capitaine à Grillon (Guilaine et al., 1988; Sauzade et al., 1990). Le site de plein air des Vignes-Saint-André à Gigondas (Bretagne et al., 1986; Jacob et al., 1987-1988) et la couche II du site de la Clairière à La Roque-sur-Pernes présenteraient avec ce groupe des ressemblances (D'Anna, 1995b, p. 276).

Cette production rassemble des récipients de toute petite taille aux formes cylindro-sphériques ou tronconiques asso- ciés à des éléments plastiques tels que des boutons. À cela s'ajoutent des vases carénés. Ces contenants sont souvent munis de mamelons prismatiques, de cordons reliant les préhensions, de cordons courts disposés en patte d'oie autour des préhensions ou de pastillage. Les décors en creux sont absents.

Cet ensemble culturel demande toutefois à être confirmé et précisé: il est en effet défini à partir d'une documentation spécifique issue d'aménagements dont la fonction est principalement sépulcrale. Le groupe Nord-Vaucluse est aussi limité géographiquement à une microrégion. Le mobilier constitue donc un échantillon très particulier et restreint. De plus, l'utilisation longue des hypogées et l'existence certaine de différentes phases de dépôt soulèvent des problèmes d'attribution de la totalité du corpus à un même ensemble culturel. Enfin, hormis la typologie céramique, les industries lithiques et osseuses, tout comme les formes de l'habitat, ne sont pas connues.

\section{LA PÉRIODISATION DE 3700 À 2200 AV. J.-C.}

\section{LE PREMIER MODÈLE}

Dans cette configuration, A. D’Anna a proposé, au début des années 1990, une périodisation du Néolithique final en deux stades généraux rassemblant chacun deux étapes pour ne plus seulement commenter des modifications plus ou moins nettes d'un aspect particulier de la culture matérielle des groupes individualisés, mais tenter d'aborder l'organisation générale de la société du Néolithique final (D’Anna, 1995a, 1995b et 1999). Pour ce faire, il s'est appuyé sur un système chronologique construit quelques années auparavant dans le Languedoc (Guilaine, Roudil, 1976; Gutherz, Jallot, 1995). Un retour sur les données de la chronométrie au travers d'une réévaluation de la cinquantaine de datations disponibles en Provence lui a permis d'établir la succession et le chevauchement des ensembles culturels (fig. 5), ainsi que leurs limites temporelles.

Dans le modèle de A. D’Anna, quatre grandes étapes chronologiques scandent le déroulement de la fin du Néolithique sur plus d'un millénaire.

De manière très schématisée, la première phase, appelée stade 1a, serait incluse entre 3700 et 3400 av. J.-C. Cette courte étape de transition avec le Chasséen correspond à la formation d'ensembles variés issus de l'éclatement du système centralisateur. Pour la désigner, l'expression 


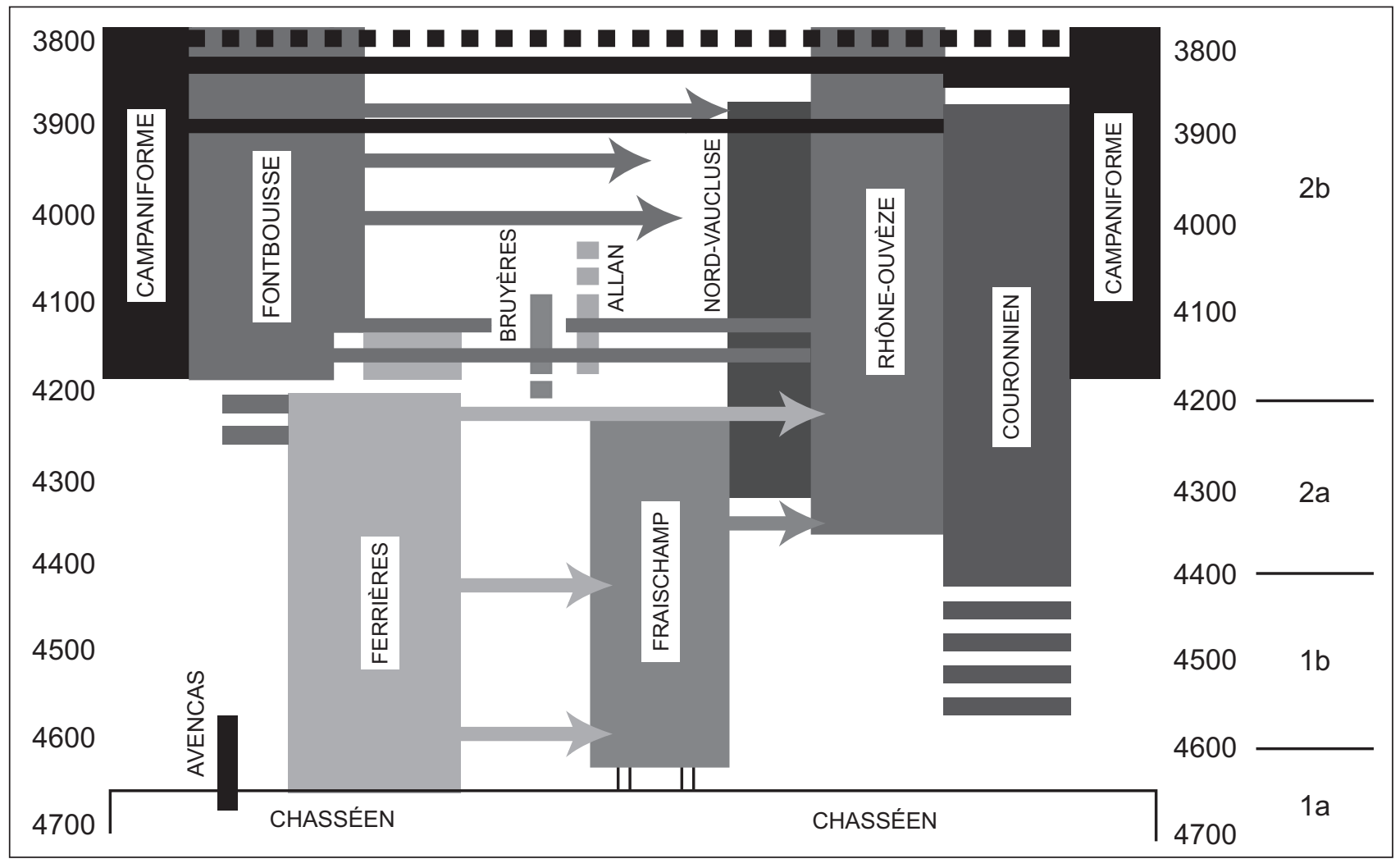

Fig. 5 - Périodisation de la fin du Néolithique en Provence. Datations BP (d'après D’Anna, 1995a et 1999).

«Néolithique récent ${ }^{2}$ » a été proposée (Gutherz, 1980a). En Provence, le mobilier de la grotte Goulard à Ménerbes dans le Vaucluse pourrait en représenter un jalon (Sauzade, 1990b; Jacob et al., 1990).

La deuxième phase, nommée stade $1 b$, se calerait entre 3400 et 2900-2800 av. J.-C. Elle serait marquée par l'apparition du groupe de Fraischamp et, plus tard, aux alentours de 3100 av. J.-C., par celle du groupe couronnien.

A. D'Anna propose ensuite une troisième phase, le stade 2a, à laquelle il attribue l'intervalle de 2800-2400 av. J.-C. Dans cette étape, une mutation s'amorcerait et produirait une transformation du fonds culturel, mise en parallèle avec le brusque déploiement de la métallurgie du cuivre à l'ouest du Rhône (Guilaine, 1994). En Provence, pendant cette période, les groupes Rhône-Ouvèze et Nord-Vaucluse se mettraient en place et le Couronnien se maintiendrait, au moins dans un premier temps.

2. «Néolithique récent» correspond à un vocabulaire d'attente. La période peut être aussi qualifiée, selon les chercheurs, de Néolithique moyen final ou tardif, de premier Néolithique final, de phase formative du Néolithique final ou de Néolithique final 0 (Lemercier, 2007).
Il définit enfin une dernière phase qui matérialiserait le quatrième temps fort de la période: il s'agit du stade 2b placé entre 2800-2700 et 2300 av. J.-C. Cet épisode est mis en rapport avec le phénomène Campaniforme qui viendrait totalement bouleverser le paysage culturel au moment où les groupes locaux sont en plein épanouissement et où fleurissent les premières cultures de l'âge du Bronze ancien. Le processus culturel campaniforme, qui affecte par ailleurs toute l'Europe de l'Ouest, se traduit par la présence dans des tombes et plus rarement dans certains habitats, d'une céramique en forme de cloche décorée de bandes ornées, parfois accompagnée de poignards en cuivre, de boutons, de rondelles en os ou d'éléments de parure. La vaste répartition de ces produits campaniformes serait aujourd'hui considérée comme le signe d'un éclatement des frontières culturelles et de la circulation affermie d'objets socialement valorisés. Dans ce stade, A. D'Anna isole deux étapes: la première où plusieurs établissements du sud de la France semblent valider l'hypothèse d'une acculturation des cultures autochtones au Campaniforme, et la seconde où la culture campaniforme authentique rhodano-provençale se met en place dans la région. Cette 


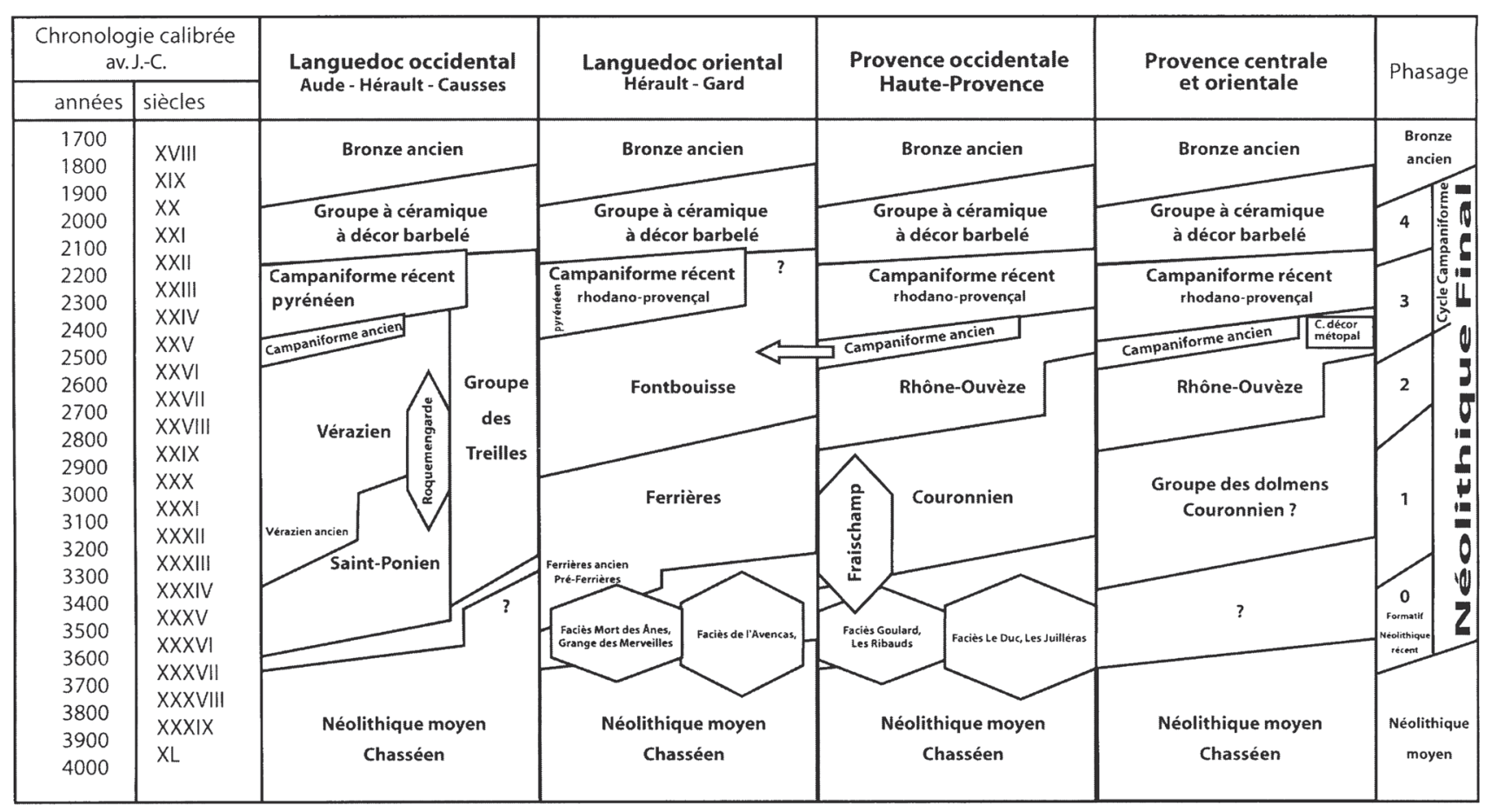

Fig. 6 - Périodisation schématique des groupes culturels de la fin du Néolithique en France méditerranéenne. Datations calibrées av. J.-C. (d'après Lemercier, 2007).

étape est caractérisée par la présence de vases décorés et d'une vaisselle domestique. Les groupes locaux ont alors complètement disparu.

\section{LES RÉAJUSTEMENTS RÉCENTS}

Dix années après la proposition de A. D’Anna, les travaux sur la périodisation sont toujours d'actualité. Depuis 1998, le Projet collectif de recherche sur le Couronnien, ainsi que plusieurs investigations sur le Campaniforme du sud-est (Lemercier, 2004a; Furestier, 2005) et sur le RhôneOuvèze (Cauliez, 2001a, 2001b et à paraître a) se sont en effet attachés à mieux définir l'insertion des groupes dans la périodisation provençale. À partir de ces travaux, O. Lemercier construit un schéma dans lequel les cultures se développeraient, au Néolithique final, non plus en quatre étapes, mais en cinq (fig. 6) (Lemercier, 2007).

L'immédiat post-chasséen pose encore des difficultés. Faute de documentation suffisante, un stade 0 (encore désigné comme «Néolithique récent») correspondrait à la composition d'ensembles variés préfigurant le Néolithique final dans la période 3700-3400 av. J.-C.
Un stade 1 se rapporterait à l'apparition de groupes territorialement bien marqués, comme celui de Fraischamp (de 3500-3400 à 3000-2900 av. J.-C.) et celui, un peu plus récent, du Couronnien (de 3400-3200 à 2800-2700 av. J.-C.) .

Le stade 2 serait une période de transformation importante qui concorderait, en Provence, avec la formation du Rhône-Ouvèze, dont l'intervalle chronologique s'étendrait de 2900 à 2500-2400 av. J.-C. Pendant cette phase, le Couronnien disparaîtrait rapidement.

Le stade 3 du Néolithique final correspondrait dans le Midi méditerranéen à l'émergence et au développement du Campaniforme, entre 2500 et 2400 av. J.-C. À l'est du Rhône, les premiers gobelets (styles standard et pointillé géométrique) apparaissent au sein d'assemblages faisant référence aux groupes Rhône-Ouvèze, généralement sur des sites de topographie particulière et géographiquement localisés sur le littoral et en bordure du Rhône. Dès ces premières phases, des transferts techniques sont remarquables entre ces ensembles culturels locaux et étrangers, tandis que des gobelets campaniformes sont diffusés loin à l'intérieur des terres. Entre 2400 et 2300 av. J.-C., le groupe campaniforme récent régional du Midi (ou Campaniforme 
rhodano-provençal) se mettrait en place. Ce groupe tendrait à remplacer les cultures locales du Néolithique final, par déculturation amorcée dès les premiers temps de la présence campaniforme.

Enfin, l'apparition des céramiques à décor barbelé et des premiers petits objets de bronze dans le Midi marquerait la fin du Néolithique entre 2200 et 2100 av. J.-C. Il s'agit du stade 4 de la périodisation d'O. Lemercier. Ces apports d'origine orientale (Italie septentrionale, nord-ouest des Balkans) s'insèrent dans l'ensemble de la région considérée et au-delà en remontant la vallée du Rhône, pour ne disparaître qu'entre 1900 et 1800 av. J.-C. avec le développement $\mathrm{du}$ plein âge du Bronze ancien et de nouveaux influx, rhodaniens cette fois. Les fortes traditions campaniformes encore sensibles conduisent $\mathrm{O}$. Lemercier à considérer cette période comme partie intégrante du Néolithique final en même temps que comme les prémices de l'âge du Bronze. Les cultures locales du Néolithique final auraient cependant disparu.

\section{LES PREMIÈRES INTERROGATIONS DÉGAGÉES DU SCHÉMA GLOBAL}

De manière générale, excepté les productions céramiques, peu d'éléments matériels permettent d'affiner les distinctions culturelles. Actuellement, des études sont en cours sur les autres composants du système technique - industrie lithique (Piatscheck, 2004 et 2005) ou sur les matières dures animales -, pour pouvoir démêler les groupes, rendre compte et interpréter les similitudes ou les divergences observées et finalement modifier l'impression d'assemblages parfois relativement similaires. Les données sont également lacunaires pour l'habitat ou les sépultures. L'habitat est particulièrement documenté pour le Couronnien, mais à peine connu pour les autres groupes. Quant aux modes funéraires, ils sont toujours définis sur le seul Nord-Vaucluse sans être recensés pour le reste des ensembles. En outre, les groupes ont été conçus uniquement sur le résultat acquis par l'observation empirique du mobilier et non pas sur des données quantitatives ou statistiques. Le nombre de sites rattachés aux différents groupes culturels et sur lesquels se fondent les définitions est aussi très inégal. D’un côté, des effectifs importants sont disponibles pour le Couronnien et le Rhône-Ouvèze. De l'autre, la caractérisation du Fraischamp et du Nord-Vaucluse est établie sur une moyenne d'environ cinq établissements. Les sites livrent par ailleurs des informations de qualité très variable. Peu de fouilles exhaustives viennent alimen- ter le débat, alors que la plupart des groupes culturels ont été caractérisés sur la base de petites opérations, parfois anciennes et pour beaucoup inédites. Cela est d'autant plus vérifiable pour le Fraischamp et le Nord-Vaucluse. Quant au Rhône-Ouvèze, la définition princeps repose sur la céramique recueillie dans seulement trois fosses explorées lors de la fouille préventive de la Plaine des Blancs. Un déséquilibre dans la documentation dont on dispose pour chacun des groupes est donc visible.

On retiendra par ailleurs de ce premier aperçu que cette mosaïque culturelle du III $^{\mathrm{e}}$ millénaire se présente comme inscrite dans un espace microrégionalisé. Trois des quatre cultures provençales sont définies dans le Vaucluse: le Fraischamp, le Rhône-Ouvèze et le Nord-Vaucluse. De plus, le Fraischamp et le Nord-Vaucluse ont une extension limitée à ce département. Les connaissances acquises paraissent donc encore peu favorables pour comprendre les imbrications des groupes de la fin du Néolithique sur de vastes espaces géographiques.

Les deux modèles de périodisation énoncés par A. D'Anna et O. Lemercier soulèvent également quelques remarques. Malgré la mise en place d'une nouvelle périodisation par O. Lemercier, qui reprend finalement dans les grandes lignes les travaux originaux de A. D'Anna, les différences introduites rendent cette problématique encore plus touffue. D'abord, les dates retenues pour les transitions fluctuent énormément. L'arrivée du Campaniforme dans le Midi méditerranéen varie entre une hypothèse haute vers 2800-2700 av. J.-C. dans le schéma de A. D’Anna et une hypothèse basse vers 2500-2400 av. J.-C. chez O. Lemercier. Il en est de même pour les limites ante quem données au Néolithique final. Dans la première périodisation, les bornes se situent après l'arrivée du Campaniforme récent et dans la seconde, après les premiers Campaniforme Bronze ancien barbelé. L'étape finale de la périodisation de O. Lemercier considère aussi que le Campaniforme barbelé succède au Campaniforme rhodano-provençal dans le sud-est de la France. C'est là un enchaînement qui mérite d'être débattu dans le sens où, si cette piste tire toute son essence du modèle princeps de J. Guilaine (Guilaine, 1967; Guilaine et al., 2001; Lemercier, 2003), elle n'a jamais pour autant été strictement vérifiée (Vital, 2005a, p. 17-23). Toujours en termes de successions chronoculturelles, des dissemblances existent aussi dans le calage du groupe Rhône-Ouvèze par rapport au Couronnien, qui varie suivant les auteurs, entre un parallélisme important en conformité avec les datations ${ }^{14} \mathrm{C}$ disponibles, ou le remplacement d'un groupe par un autre. 
En définitive, dans le canevas de A. D'Anna, le Couronnien perdure loin dans le stade 2a matérialisé par les groupes Rhône-Ouvèze et Nord-Vaucluse, tandis que dans celui de O. Lemercier, le Couronnien disparaît rapidement pour laisser place au Rhône-Ouvèze dans l'étape 2 (Guilaine et al., 2001). Le groupe du NordVaucluse, généralement attribué à cette même phase, n'est pas non plus repris dans la seconde périodisation en raison des doutes portant sur sa définition. Celui-ci pourrait, selon O. Lemercier, «correspondre à plusieurs ensembles chronologiques distincts se rapportant à d'autres cultures définies» (Lemercier, 2007, p. 496). Le problème du Nord-Vaucluse reste donc entier, même si dans cette périodisation il est totalement ignoré. À cela s'ajoute le fait que les dates choisies (D’Anna, 1995b, fig. 8; Guilaine et al., 2001, annexe III; Lemercier et al., 2007, fig. 4), distinguant chacun des échelons dans les deux périodisations, n'ont qu'une valeur indicative et de commodité. Même si elles offrent aux auteurs la possibilité, après calibration, de proposer des repères chronologiques, elles demeurent pour beaucoup assorties de marges d'erreurs relativement importantes. On se heurte au problème du manque de datations, mais aussi à l'utilisation de datages parfois inexploitables dans l'élaboration d'une chronologie absolue; l'élément daté provient de contexte imprécis ou les datations obtenues sont accompagnées de grands écarts-types. La question de la chronologie est de taille, car dans cette période se déploient des phénomènes majeurs comme la fin du Chasséen, l'apparition de la métallurgie, l'arrivée et le développement du Campaniforme, la mise en place des premières communautés de l'âge du Bronze.

\section{LA QUESTION DE LA MISE EN PLACE ET DES ORIGINES DES GROUPES PROVENÇAUX}

\section{LA CONSTITUTION D'UNE SOURCE D'INSPIRATION}

Le panorama suivant rappelle les étapes successives dans la constitution des cadres chronoculturels au travers d'un bilan historique sur le concept d'âge du Cuivre et sur le rôle de la céramique dans l'élaboration du système chronoculturel en usage dans le midi de la France. Il s'en dégage qu'à chaque stade d'avancement de la recherche, la fin du Néolithique en Languedoc constitue la référence pour comprendre et observer la mise en place et la succession des ensembles culturels de la rive gauche du Rhône (fig. 5 et 7).

\section{MiSE EN PLACE DU CHALCOLITHIQUE ET RÔLE DU CUIVRE}

\section{La position princeps du Languedoc}

La recherche sur la fin du Néolithique s'apparente à une priorité restée longtemps secondaire. En effet, cette période a été abordée dans un premier temps à l'échelle nationale pour combler des vides dans un cadre chronoculturel global en cours de formation. Au moment où J. Arnal, G. Bailloud et M. Escalon de Fonton innovent en matière de Néolithique hexagonal dans quelques articles sur les premiers systèmes néolithiques, le fer de lance de la recherche reposait pleinement sur certaines thématiques privilégiées comme la néolithisation et le Néolithique moyen (Arnal, 1953; Arnal, Burnez, 1958; Arnal, Prades, 1958; Arnal et al., 1960 ; Bailloud, Mieg de Boofzheim, 1955; Escalon de Fonton, 1955, 1958 et 1963). Les problèmes de la définition de ce qui était postérieur au Néolithique moyen chasséen et qui précédait l'âge du Bronze sont abordés plus franchement dans des problématiques spécifiques lorsque les recherches se concentrent sur la question des âges des métaux. Cette conception des choses donne à la fin du Néolithique un statut de passage, sans que l'on en apprécie la valeur culturelle, pourtant traduite par des éléments matériels difficilement classables dans les phases antérieures ou postérieures.

Comment définir alors cette zone d'incertitude chronologique comprise entre un Chasséen finissant et un âge du Bronze ? Très tôt, le cuivre apparaît comme l'élément structurant dans l'organisation de la période, avec comme débat sous-jacent celui de savoir si ce moment de la Préhistoire constitue encore un plein Néolithique ou s'inscrit déjà dans l'âge du Bronze. La structuration chronologique, géographique et culturelle du Midi méditerranéen n'échappe pas à ce mouvement et se construit autour de ce repère, rapidement identifié dans la région languedocienne considérée aujourd'hui à l'origine de la première métallurgie du cuivre en France (Ambert et al., 2005). C'est aussi en Languedoc, à la fin du $\mathrm{XIX}^{\mathrm{e}} \mathrm{s}$. et au début du XX $\mathrm{X}^{\mathrm{e}}$ s. av. J.-C., que les travaux sur cette période sont les plus foisonnants, car la richesse exceptionnelle des découvertes dans ce secteur géographique, où les prospections, les fouilles et les publications se multiplient, en fait un important pôle scientifique sur la Préhistoire récente (Guilaine, 1972). M. Louis d'abord et J. Arnal et J. Audibert ensuite délimiteront ainsi les contours du premier paysage culturel de la fin du Néolithique dans le midi de la France, non seulement à partir de stratigraphies dilatées permettant de décortiquer 


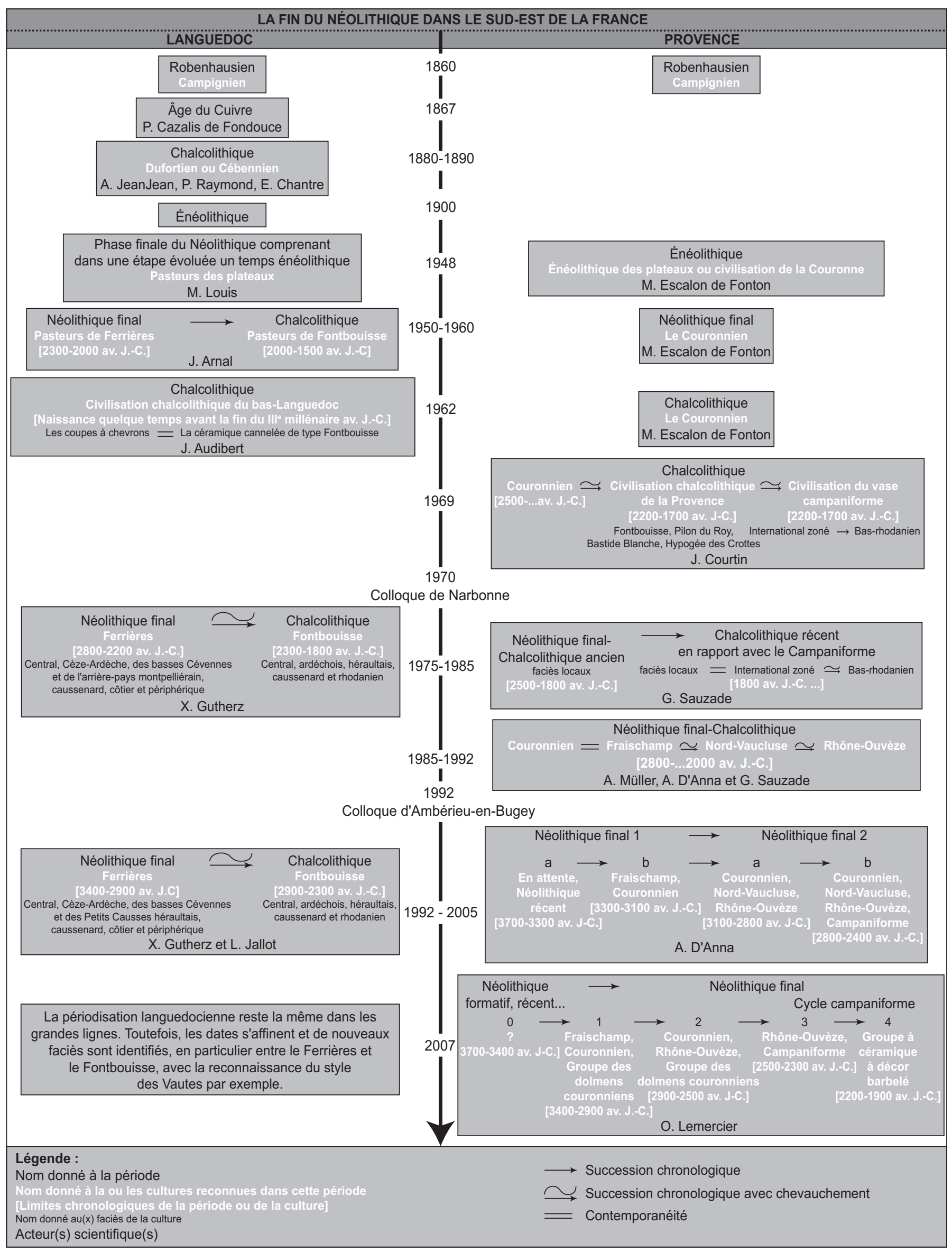

Fig. 7 - Évolution concomitante de la trame chronoculturelle languedocienne et provençale depuis la fin du XIX s. à aujourd'hui. Le rôle du cuivre et de la céramique (DAO: J. Cauliez). 
dans le temps long l'évolution du Néolithique, mais aussi grâce à la fouille extensive d'importants sites de plein air.

La reconnaissance de l'existence dans le sud de la France d'un âge du Cuivre entre le Néolithique et l'âge du Bronze est ancienne (fig. 7); il faut remonter au XIX ${ }^{\mathrm{e}} \mathrm{s}$. av. J.-C. pour trouver les premières références dans les travaux de 1867 menés par P. Cazalis de Fondouce. Celui-ci met en évidence la présence du métal sur certains sites languedociens (Cazalis de Fondouce, 1867). P. Raymond, A. Jeanjean et E. Chantre prennent la suite dans les années 1880-1890 et défendent pareillement l'idée d'une période intermédiaire qu'ils dénomment alors «Chalcolithique». Le Chalcolithique est défini comme un état de civilisation caractérisé par l'usage simultané, pour l'outillage et l'armement, de la pierre (silex et roches assimilées) et du cuivre (Chantre, 1875-1876; Jeanjean, 1884). Mais la réalité est plus complexe, et à ce moment de la recherche la subdivision Néolithique/Chalcolithique/âge du Bronze est parfois mise en doute par certains préhistoriens, d'abord par P. Cazalis de Fondouce lui-même qui considère finalement le Chalcolithique comme un stade particulier du Néolithique finissant (Cazalis de Fondouce, 1900), ensuite par J. de Saint-Venant, qui préfère inversement le rattacher au début de l'âge du Bronze (Saint-Venant, 1908).

Le terme de Chalcolithique tombe progressivement en désuétude dans les pays septentrionaux où on le remplace, dès le début du $\mathrm{XX}^{\mathrm{e}}$ s., par celui d'«Énéolithique» calqué sur un terme italien. L'Énéolithique éclipsera par son rayonnement les âges qui l'encadrent. Nombreux sont en effet les chercheurs convaincus d'une brillante civilisation énéolithique qui, succédant au Néolithique, aurait évolué sur place, parfois même jusqu'à l'âge du Fer, sans grandes modifications. Les auteurs méridionaux, tels que Th. et Ph. Héléna, F.-Ch.-E. Octobon ou plus tard G. Fabre, contestent en fait l'existence d'un véritable âge du Bronze car, pour cette période, les découvertes métalliques, dans le midi de la France, sont moins nombreuses que dans les régions du Nord, de l'Ouest et de l'Est (Guilaine, 1972, p. 15; Roudil, 1972; Vital, 2005b, p. 24). Quelques années après la définition d'une brillante civilisation énéolithique, M. Louis revient sur cette subdivision. Dans le système chronoculturel complexe qu'il élabore et publie en 1948, il introduit un «état de civilisation énéolithique» correspondant à une phase finale du Néolithique et dans laquelle le métal s'infiltre timidement (Louis, 1948, p. 76). À contrecourant de cette proposition, J. Arnal prend à son tour clairement position dans le milieu des années 1950, en soutenant l'hypothèse d'un Chalcolithique, que l'on peut identifier sur les sites attribués au groupe de Fontbouisse du fait de la présence d'objets en métal, lequel précède le Bronze ancien et succède à un Néolithique final rattachable au groupe culturel Ferrières. La fin du Néolithique est donc subdivisée en deux temps forts, caractérisés par la présence ou non du cuivre dans les cultures (Arnal, 1953; Arnal, Burnez, 1958; Arnal, Prades, 1958; Arnal et al., 1960).

Ces premiers jalons s'achèvent par les travaux de J. Audibert qui choisit pour sa part d'apporter un modèle différent (Audibert, 1958 et 1962). Dans sa conception de la période, les œuvres de A. Jeanjean et de P. Raymond (1897 et 1898) sont réhabilitées par la reprise du terme de Chalcolithique, dont J. Audibert retient le concept "d'état de civilisation", auquel il assigne une dimension chronologique large: le Chalcolithique bas-languedocien constitue une période clé du Néolithique méridional, dans laquelle il revalorise l'impact de la métallurgie. Le Chalcolithique rassemble alors les deux phases établies plus tôt par J. Arnal.

\section{Le suivisme provençal}

En Provence, les dernières décennies du $\mathrm{XIX}^{\mathrm{e}}$ s. et les premières années $d u X X^{e}$ s. jusqu'à quelques années après la Seconde Guerre mondiale sont marquées par les investigations de chercheurs tels que S. Gagnière et M. Paccard pour le Vaucluse, A. Taxil pour le Var, M. Escalon de Fonton pour les Bouches-du-Rhône et l'équipe du musée d'Anthropologie préhistorique de Monaco dirigée par L. Barral pour les Basses-Alpes et les Alpes-Maritimes (Buisson-Catil et al., 2004; Müller et al., 2005; Delestre, Buisson-Catil, 2006). Malgré de nombreuses recherches orientées vers les sites en grottes ou les abris, la mise en évidence de stratigraphies valides permettant de formuler, pour la première fois, une structure du Néolithique provençal, s'avère délicate. La Préhistoire récente de la région évolue donc de concert avec le Languedoc, en s'appuyant très vite sur les divers consensus développés autour du cuivre par la communauté archéologique ouest-rhodanienne, indépendamment du fait qu'il n'y a pas, en Provence, de métallurgie (fig. 7). De 1954 à 1963 par exemple, dans la tentative de restitution de la fin des temps néolithiques provençaux par M. Escalon de Fonton, la première culture qu'il définit, le Couronnien, est d'abord nommée «Énéolithique des plateaux» en référence à M. Louis (Escalon de Fonton, 1954, p. 93). En 1963, M. Escalon de Fonton attribue ensuite le Couronnien au Chalcolithique ancien en l'inscrivant dans le contexte languedocien décrit à l'époque par J. Audibert (1958 et 1962). 
Alors que M. Escalon de Fonton poursuit sa lancée et étaye sa définition du Couronnien, J. Courtin apporte à son tour de nouveaux éléments à un schéma encore mal documenté qu'il choisit de structurer, lui aussi, autour de la notion de Chalcolithique. Les avancées, aussi significatives soient-elles, ne sont pas suffisantes pour permettre à cette époque d'entrevoir un Néolithique final moins unifié à l'échelle de l'arc méditerranéen et une Provence non annexée au Languedoc. Dans sa thèse de doctorat intitulée Le Néolithique de la Provence: Préhistoire de la Provence à l'Holocène du Néolithique au début de l'âge du Bronze soutenue en 1969, J. Courtin adopte par conséquent, comme M. Escalon de Fonton, le terme de Chalcolithique employé dans le sens de G. Bailloud (1961) et J. Audibert (1962) pour désigner le Néolithique finissant, tout en expliquant qu'en Provence, il n'existe pas de véritable âge du Cuivre tel qu'il a pu être mis en évidence à l'ouest du Rhône. Cet emprunt au Languedoc «présente en fait l'avantage de traduire le caractère ambigu de cette période transitoire entre le Néolithique et l'âge du Bronze» (Courtin, 1974, p. 215). J. Courtin concède au Chalcolithique à la fois une acception élargie, saisie comme un stade culturel qui va de la fin du Chasséen au Bronze ancien, mais aussi une valeur chronologique générale: cet épisode débute dans la deuxième moitié du III $^{\mathrm{e}}$ millénaire av. J.-C. et s'achève vers 1600 av. J.-C. Pour cet intervalle, J. Courtin différenciera trois civilisations: celle du Couronnien, relayée par la «civilisation chalcolithique de la Provence» appelée aussi «Chalcolithique provençal», suivie par la «civilisation du vase campaniforme».

\section{Néolithique final ou Chalcolithique: le débat du colloque de Narbonne}

En 1970, une discussion est entamée sur la notion de Chalcolithique au cours du colloque tenu à Narbonne sur Les Civilisations néolithiques du midi de la France (Guilaine, 1970). Elle débouche sur un réajustement des différents points de vue car, dans les recherches du moment, le Chalcolithique semble bien avoir deux significations discordantes (fig. 7). Il peut matérialiser d'une part, le Néolithique finissant dans une acception et une chronologie étendues (un état de civilisation) et d'autre part, relever d'un sens plus réduit comprenant exclusivement l'extrême fin des temps néolithiques associée à la métallurgie. Il en découle des modèles chronoculturels de plus en plus équivoques: concrètement, les mêmes termes sont utilisés pour pointer des phénomènes différents. À l'occasion du colloque, il est par conséquent décidé que «Chalcolithique» qualifiera désormais non plus une période chronologique, ni un état de civilisation, mais une modification d'ordre technologique; il faut l'employer «pour désigner [...] les cultures qui connaissent la métallurgie du cuivre», et seront placées au Néolithique final «celles qui ne la pratiquent pas» (ibid., p. 127). Le système chronologique utilisé en Languedoc demeure inchangé et se réserve l'emploi du terme Chalcolithique pour les cultures qui ont pratiqué la métallurgie de façon certaine. Au milieu des années 1980, X. Gutherz adopte ces propositions et confirme, à la suite de J. Arnal, une succession chronoculturelle languedocienne en deux étapes, Néolithique final Ferrières, puis Chalcolithique Fontbouisse (Gutherz, 1980b, 1982 et 1984). C'est aussi le moment où il est clairement avéré que la première métallurgie du cuivre, sur la rive droite du Rhône, est précampaniforme (Gutherz et al., 2005, p. 120).

\section{Le «cuivre", un critère inadapté dans la construction des cadres provençaux}

L'inadaptation du critère «cuivre» dans le maintien des cadres provençaux est décelable de manière évidente dans la thèse de doctorat de G. Sauzade sur Les Sépultures $d u$ Vaucluse du Néolithique à l'âge du Bronze qu'il soutient au sortir des années 1970 (Sauzade, 1983). Toute l'ambivalence de sa démarche réside dans le fait que le cuivre demeure peu connu de ce côté-ci du Rhône et que les recherches ne peuvent pas encore s'appuyer sur des données totalement exploitables. Dix années après la thèse de J. Courtin, la qualité des informations disponibles pour cette période - manque de fouilles, peu de sites de plein air, pas de stratigraphie valide, etc. - n'offre pas la possibilité d'aller au-delà de concepts très simplificateurs et d'une terminologie d'attente calquée sur le Languedoc (fig. 7).

À partir de sa révision systématique des ensembles funéraires vauclusiens (une centaine), G. Sauzade subdivise donc à son tour le Chalcolithique de J. Courtin en deux épisodes: un «Néolithique final-Chalcolithique ancien", puis un «Chalcolithique récent». Pour le premier stade, il ne peut que combiner des termes pourtant en inadéquation si l'on reprend les normes instaurées par le colloque de Narbonne car, comme il l'explique, il est encore difficile en Provence «à l'heure actuelle, de dissocier les cultures matérielles qui sont propres au Néolithique final de celles se rattachant au Chalcolithique ancien. Il se trouve qu'un certain nombre de gisements, les uns contenant des objets en cuivre, les autres pas, ont fourni un mobilier 
et, notamment, des motifs de décors identiques" (ibid., p. 26). À cela s'ajoute le fait que les cultures pratiquant la métallurgie et celles ne la connaissant pas peuvent parfois être contemporaines, comme l'avait soulevé J. Guilaine au colloque de Narbonne (Guilaine, 1970, p. 127). Le second stade indépendant, «le Chalcolithique récent», correspond à l'extrême fin du Néolithique, où les populations autochtones voient l'apparition et le développement du Campaniforme, ainsi que la diffusion d'objets en cuivre. Ici encore, deux pans chronoculturels dissociés plus tôt par J. Courtin sont réunis: la phase évoluée du «Chalcolithique provençal» et la «civilisation du vase campaniforme», car il précise qu'en Vaucluse les deux civilisations coexistent. Le développement des notions de Néolithique final, de Chalcolithique ancien et récent, dans un même niveau de définition, mêlant chronologie et technologie, ainsi que les problèmes du rôle et de la place du Campaniforme au sein des groupes allogènes, contribuent dès lors à un certain imbroglio dans la lecture de la mise en place des ensembles culturels.

Au début des années 1990, cette confusion perdure dans les premières propositions qui sont faites après la création des groupes Rhône-Ouvèze, Fraischamp et Nord-Vaucluse, réintégrés avec le Couronnien au sein d'un unique complexe vaste de «Néolithique final-Chalcolithique» (Müller et al., 1986; Sauzade et al., 1990). Ce large cadre chronoculturel recoupe les différents temps de la périodisation languedocienne, mais la tentative d'englober dans un même concept diverses manifestations culturelles, potentiellement indépendantes de la métallurgie, devient méthodologiquement discutable.

Le débat sur le terme de Chalcolithique est relancé au colloque d'Ambérieu-en-Bugey de 1992 (Voruz dir., 1995), où A. D’Anna suggère son abandon définitif pour la Provence au profit de celui de Néolithique final (D’Anna, 1995b). La diffusion du métal de ce côté-ci du Rhône n'est pas vraiment documentée (Courtin, 1974; Courtin, Sauzade, 1975). A. D’Anna précise «qu'il n'y a guère de données nouvelles sur ce point et l'on peut seulement rappeler qu'il n'y a probablement pas de métallurgie locale avant l'extrême fin de la période, alors que les premiers objets, poignards et parures notamment, apparaissent avant la diffusion du Campaniforme»(D’Anna, 1995b, p. 279). Toutefois, le principe d'une évolution concomitante entre le Languedoc et la Provence persiste et bien que le cuivre soit absent, le canevas chronoculturel de mise en place des groupes est à nouveau conçu à l'image du système languedocien. Dans sa périodisation, A. D’Anna subdivise en effet le Néolithique en deux étapes phares. Dans ce modèle de structuration, on retrouve la bipartition de J. Arnal reprise par X. Gutherz; les séparations dans les grandes étapes sont placées à l'identique et l'argumentaire, certes logiquement un peu différent, entre toujours dans le même mode de questionnement.

À partir des données actuelles, on considère aujourd'hui que la métallurgie du cuivre a dû démarrer modestement autour de 3000 av. J.-C. en Languedoc oriental, peut-être avant, dans un environnement culturel local finalement encore dominé par le groupe de Ferrières, puis par ses épigones (groupes de la vallée de l'Hérault, groupe des Vautes), considérés parfois comme les manifestations locales d'un Ferrières tardif à répartition large (Gutherz et al., 2005, p. 127). La preuve de l'ancienneté de la métallurgie du cuivre s'appuie en particulier sur les éléments obtenus récemment sur les aires métallurgiques de la Capitelle du Broum à Péret dans l'Hérault, qui donne une fourchette de probabilité de 3310-3230 av. J.-C. (Ambert et al., 2005). Même si la métallurgie ne s'est vraiment développée qu'après 2900-2800 av. J.-C. avec la culture de Fontbouisse, les recherches tendent à mettre en évidence que cette dichotomie entre Néolithique final Ferrières d'un côté et Chalcolithique Fontbouisse de l'autre n'est plus tellement valide. Plusieurs articles rappellent d'ailleurs les termes de ce débat en cours sur l'opportunité de maintenir ou non le vocable de Chalcolithique dans le système chronoculturel instauré dans la région languedocienne (Ambert, 1990-1991; Carozza, 2000 et 2002; Strahm, 2005; Guilaine, 2007).

En Provence, les travaux de H. Barge dans les mines de Clausis à Saint-Véran dans les Hautes-Alpes ont parallèlement mis en lumière la métallurgie à l'est du couloir rhodanien (Barge-Mahieu, 1995; Barge et al., 2005). Des nouvelles données semblent donc dessiner des constantes qui pourraient être caractéristiques, sans être exclusives, d'une métallurgie dans l'ensemble du sud-est de la France. Le travail entrepris sur la céramique de la mine préhistorique de Saint-Véran pointe cependant des affinités avec le Chalcolithique italien (Morin et al., 2005). Pour les tuyères par exemple, les référentiels renvoient au Chalcolithique de la région de Garda-Trentino. Les récipients sont à rapprocher du site de Vollein (Aoste) et sont également comparés aux céramiques grossières du Roc del Col (Usseaux, Turin), site d'altitude du Bronze moyen italien (Rossi, Gatigglia, 1998). Il reste donc difficile d'être affirmatif sur l'existence d'une métallurgie provençale au Néolithique final, du fait de la rareté des éléments de comparaison disponibles dans 
le quart sud-est. Les informations relatives à la métallurgie, à l'est de la sphère languedocienne sont en effet trop lacunaires pour constituer de véritables points de comparaison.

Cependant, bien qu'en périphérie de ce grand mouvement d'innovation technique, la totalité des groupes provençaux bénéficie du développement de la métallurgie dans les régions adjacentes par l'importation d'instruments de cuivre dès la première moitié du III millénaire av. J.-C. Il faut en effet signaler quelques découvertes comme celles des alênes et du poignard italien à lame triangulaire et à languette trouvés dans un dolmen d'Orgon dans les Bouches-du-Rhône (Courtin, Sauzade, 1975; BargeMahieu, 1995), les alênes des dolmens de Camdumy à Flassans-sur-Issole et de la Verrerie-Vieille à Tourrettes et les perles des mégalithes de Muraires au Luc et de Roque d'Aille à Lorgues, tous les quatre dans le Var (Guilaine, 1994), auxquels on attribue une origine italienne ou languedocienne, ou encore de multiples petits objets exhumés dans divers gisements, comme ceux de Basse-Provence côtière, le Collet-Redon ou Ponteau-Gare, pour n'en citer que quelques-uns. Toujours du côté italien, de toutes nouvelles études ont montré également la précocité des activités minières liées au travail du cuivre, en particulier en Ligurie orientale et ce dès le milieu du IV e millénaire av. J.-C. (Binder et al., 2008, p. 57-58). Au Monte Loreto notamment, l'exploitation des mines de cuivre est mise en parallèle avec la répartition dans les Alpes de modèles d'arme et de parure en cuivre d'origine centro-européenne. Ces pièces auraient pu aboutir jusque dans l'arc liguro-provençal, comme par exemple la pendeloque à double spirale de la grotte de Magagnosc comparée par C. Strahm aux pièces connues en contexte Pfyn (ibid.).

Au-delà de la seule importation en Provence, les objets en cuivre sont aussi intégrés dans la chaîne opératoire sous la forme de véritables outils, de type compresseur métallique, utilisés dans le débitage à la pression au levier, avant 3000 av. J.-C., pour la confection des poignards en silex du Largue dans le bassin d'Apt-Forcalquier (Renault, 2006, p. 159). Même dans le domaine des techniques céramiques, des modifications des savoir-faire au cours du Néolithique final entre la fin du $\mathrm{IV}^{\mathrm{e}}$ millénaire et la première moitié du III ${ }^{\mathrm{e}}$ millénaire av. J.-C., sont mises en rapport avec les connaissances des techniques métallurgiques (Durrenmath, 2005). Les manières de fabriquer se transforment dans les productions régionales et se traduisent en effet par un traitement du dégraissant lié à la recherche de terres à texture lâche pour la fabrication de récipients aux capacités plus réfractaires, susceptibles de supporter des températures élevées, ce qui pourrait correspondre à une plus grande maîtrise des techniques de cuisson, elle-même liée à un savoir des pratiques métallurgiques.

\section{LE RÔLE DE LA GÉRAMIQUE DANS LA GRILLE DE CONSTRUCTION DES GROUPES}

Ces dernières découvertes témoignent donc de l'antériorité de la métallurgie du cuivre en Languedoc dès le Ferrières, de la pénétration d'objets cuivreux dans la culture matérielle provençale, de modifications techniques liées à son avènement dans cette région et d'une métallurgie italienne en Provence à des dates très basses (Guilaine, Vaquer, 1976), mais cela ne réfute pas pour autant la validité des cadres chronoculturels ébauchés ces soixante dernières années. La structure du Néolithique final s'est en effet bâtie simultanément par les études portant sur la céramique régionale. C'est d'ailleurs, une fois de plus, en Languedoc qu'il est possible de recueillir les éléments de comparaison les plus nombreux et les mieux documentés. J. Arnal a, dès le début des années 1950, fait correspondre à chacun des deux grands stades définis par la présence ou non de métallurgie, des productions céramiques dans lesquelles des distinctions stylistiques et typologiques très nettes lui ont permis de différencier à son tour un Néolithique final Ferrières, puis un Chalcolithique Fontbouisse (fig. 7) (Arnal, 1953).

En référence aux travaux des préhistoriens ouest-européens du début du XX ${ }^{\mathrm{e}}$ s., J. Arnal est le premier en France à utiliser la céramique dans sa dimension chronologique comme traceur culturel: jusqu'alors, les chercheurs de l'hexagone persévéraient dans les voies de la classification exclusivement lithique. On lui doit aussi les premières tentatives de structuration du Néolithique méditerranéen (Guilaine, Gutherz, 1990), car celui-ci saisit en effet, à la suite des travaux de L. Bernabò Brea sur les Arene Candide (Bernabò Brea, 1956), qu'une stratigraphie dilatée permet de décortiquer dans le temps long l'évolution du Néolithique. Ces deux avancées lui donnent des bases plus fiables à son schéma évolutif. À cela s'ajoute le fait que des progrès décisifs ont également lieu au sortir de la Seconde Guerre mondiale: ils se traduisent par la multiplication des fouilles stratigraphiques et le développement des méthodes de datation radiocarbone. Pour le Ferrières, comme pour le Fontbouisse, des dates sont obtenues et partitionnent la fin du Néolithique en deux temps successifs.

J. Arnal propose ainsi pour le sud de la France sa subdivision chronoculturelle de la civilisation des pasteurs des 
plateaux en ce temps-là représentative, selon M. Louis, de la partie terminale des temps néolithiques (Louis, 1948; Arnal, 1953; Arnal, Burnez, 1958; Arnal, Prades, 1958; Arnal et al., 1960). À l'intérieur de ce grand ensemble, J. Arnal reconnaît les deux cultures de Ferrières et de Fontbouisse, à partir de la céramique, mais aussi en tenant compte de l'association des différents produits manufacturés, tels que l'outillage en silex, en os ou la parure. La première civilisation, nommée alors «pasteurs des plateaux, variété de Fontbouisse", est caractérisée en rapprochant la céramique (décors de cannelures, disposition en métopes, formes carénées) de l'habitat de Fontbouisse à Villevieille dans le Gard (Peyrolles, Louis, 1946), à plusieurs ensembles issus des grottes du Gardon (Peyrolles, Arnal, 1954). Un peu plus tard, en 1956, J. Arnal identifie, à partir des décors et des formes céramiques (cannelures, disposition en chevrons) du dolmen de Ferrières-les-Verreries dans l'Hérault une seconde culture, "les pasteurs de Ferrières", reconnue, en s'appuyant sur les stratigraphies de la grotte de la Madeleine (Villeneuve-lès-Maguelone, Hérault) et de l'aven de Suquet-Coucolière (Les Matelles, Hérault), comme plus ancienne que les pasteurs des plateaux, variété de Fontbouisse (Arnal, 1956 et 1963).

Aujourd'hui, ces classifications en matière de groupes typologiques, reprises et précisées dans les années 1970 et 1980 par R. Montjardin, J. Gasco et X. Gutherz (Montjardin, 1965 et 1966; Gasco, 1976; Gutherz, 1975 et 1984), restent opérantes dans leurs grandes lignes. Le Ferrières et le Fontbouisse occuperaient une plage chronologique d'environ un millénaire, la plupart des dates s'inscrivant dans une période comprise entre 3500 et 2500 av. J.-C.

Le groupe de Ferrières est inclus dans un intervalle allant de 3500-3400 à 2900 av. J.-C. On admet que cet ensemble culturel s'est constitué à partir d'une évolution relativement lente des types céramiques depuis le Chasséen final. Entre ce Chasséen et les premiers groupes de la fin du Néolithique, l'existence d'une période de transition, le Néolithique récent, a été révélée par divers travaux (Gutherz, 1980a). Il s'agit d'un stade de mutation des cultures régionales au moment de la dislocation du bloc chasséen. L'hypothèse est confortée par la mise au jour de plusieurs ensembles montrant le maintien de formes céramiques chasséennes, comme la coupe en calotte ou la coupe carénée, ainsi que l'apparition de nouveaux décors plastiques (cordons, pastillage) et de moyens de préhension telles que les prises plates sur des formes simples cylindriques, sphéroïdales ou ovoïdes. L'aven sépulcral de la Boucle à Corconne dans le Gard, la Baume de Ronze à Orgnac-l'Aven et la grotte
Saint-Marcel en Ardèche, l'habitat de la Mort des Ânes à Villeneuve-lès-Maguelone dans l'Hérault, mais aussi l'abri de Font-Juvenal à Conques dans l'Aveyron, l'abri de SaintÉtienne-de-Gourgas et la grotte Tournié à Pardailhan dans l'Hérault livrent certains niveaux attribués à ce Néolithique récent. C'est à partir de ce fonds régional relativement diversifié que s'affirmerait ensuite le groupe de Ferrières (Gutherz, Jallot, 1995, p. 232).

Le Ferrières s'étend dans tout le Languedoc oriental, de l'Ardèche à l'Hérault, et, si les recherches actuelles confirment le caractère fortement unitaire de ce groupe stylistique (Giligny et al., 1997; Jallot, 2003), il semble néanmoins se distribuer sur la base de traits céramiques univoques - combinaisons décoratives récurrentes et fréquence de certaines formes - en cinq faciès régionaux: faciès central, faciès Cèze-Ardèche, faciès des basses Cévennes et de l'arrièrepays montpelliérain, faciès caussenard et faciès côtier (voir infra, note 3).

Le groupe de Fontbouisse se développe, quant à lui, entre 2900-2800 et 2500-2400 av. J.-C. Il présente un nombre de faciès identique qui se superposent à ceux du Ferrières avec de très faibles distorsions, en conservant souvent les mêmes critères distinctifs (Gutherz, 1990 et 1990-1991). Bien que l'éventail des formes et des décorations des productions fontbuxiennes doive beaucoup à l'héritage Ferrières, il s'en éloigne cependant par la variété des types morphologiques et la richesse de l'ornementation. À propos du passage du Ferrières au Fontbouisse, de nombreux arguments - continuité stratigraphique dans des sites en grottes, répartition spatiale identique, héritage du répertoire des formes et du répertoire décoratif - permettent à plusieurs auteurs de soutenir qu'il existe une filiation directe entre les deux groupes (Jacobieski, 1988; Gutherz, Jallot, 1995, p. 242; Jallot, 2003, p. 246).

Pour la Provence, les travaux sur la céramique ont suivi le même cheminement que celui emprunté par les archéologues languedociens: bien que la différentiation des deux phases s'est construite en comparaison avec la trame chronoculturelle ouest-rhodanienne, elle s'est bâtie aussi autour de la reconnaissance d'ensembles culturels variés à partir de productions céramiques discriminantes (fig. 7). De la même manière, une série de datations, certes restreintes mais cohérentes, a par ailleurs offert aux chercheurs provençaux la possibilité d'intégrer les groupes de Fraischamp et du Couronnien dans la première étape de la périodisation et ceux de Rhône-Ouvèze et Nord-Vaucluse dans le second épisode (voir supra «La périodisation de 3700 à 2200 av. J.-C.», p. 253). 


\section{LES ORIGINES DES GROUPES GULTURELS PROVENCAUX ET LE RÉFÉRENT LANGUEDOCIEN}

Depuis les premières propositions émises dans les travaux précurseurs de M. Escalon de Fonton sur la formation du Couronnien jusqu'aux pistes actuellement développées sur la genèse du Fraischamp, du Rhône-Ouvèze et du NordVaucluse, la démonstration suivante montre non seulement la primauté languedocienne dans les schémas de mise en place des groupes, mais elle dégage également les lignes interprétatives et donc les liens plus ou moins forts dans les relations envisagées entre les cultures de part et d'autre du Rhône (fig. 8).

\section{PREMIÈRES HYPOTHÈSES SUR L'ORIGINE DU COURONNIEN : L'ÉCHO DU LANGUEDOC}

Au moment de la création du Couronnien, un cadre général permettant une meilleure appréciation de la fin du Néolithique dans sa globalité faisait toujours défaut (D’Anna, 1995a). M. Escalon de Fonton n'a pu par conséquent se raccorder à aucun canevas chronoculturel. Sans vraiment proposer une interprétation de la civilisation de la Couronne, il en ébauche néanmoins une à partir d'une comparaison avec le Chasséen dans sa définition du moment. Pour lui, cette civilisation est originale: «...nous voyons une industrie lithique bien spéciale et son contexte céramique bien particulier (qui n'est ni chasséen, ni Lagozza, ni almérien), que l'on reconnaît au premier coup d'œil lorsqu'on prospecte les plateaux calcaires qui bordent la côte» (Escalon de Fonton, 1954, p. 95). La définition princeps du Couronnien, qui se fait donc en fonction de critères négatifs par rapport au Néolithique moyen chasséen, constitue un exemple remarquable du manque de repères chronologiques et géographiques à l'époque des recherches. M. Escalon de Fonton est contraint, pour trouver rapidement au Couronnien des points d'ancrage plus en adéquation avec la fin du Néolithique, d'envisager son rattachement à des complexes culturels hors du cadre régional et en particulier languedociens. Il s'inspire donc directement des recherches successives de M. Louis, J. Arnal et J. Audibert (fig. 8).

C'est ainsi qu'au fil des années, M. Escalon de Fonton assimile le Couronnien à chacune des cultures languedociennes (Durrenmath et al., à paraître). En 1954, le Couronnien, nommé tantôt «Énéolithique des plateaux»en référence à M. Louis, tantôt «civilisation de la Couronne» «offre certaines analogies avec ce que Maurice Louis désigne sous le nom de "pastoral campignien" du Languedoc» (Escalon de Fonton, 1954, p. 93). Si, en 1956, le terme Couronnien est officialisé (Escalon de Fonton, 1956), cette appellation est complétée par la mention "Énéolithique des plateaux" (ibid., p. 132). En 1963, M. Escalon de Fonton attribue le Couronnien au Chalcolithique ancien en l'inscrivant dans le contexte languedocien décrit à l'époque par J. Audibert (1958 et 1962): "Le Couronnien est contemporain du Chalcolithique du Languedoc qui, lui, possède une céramique bien décorée...» (Escalon de Fonton, 1963, p. 179). Plus tard, en 1968, il compare le décor de doubles cordons courts verticaux de la Couronne à des ornementations propres aux «stations des inconnus des Corbières» (ou Véraziens) (Escalon de Fonton, 1968, p. 56).

Dans cet ouvrage, M. Escalon de Fonton formulera toutefois une hypothèse alternative plus anecdotique selon laquelle la civilisation couronnienne résulterait d'une colonisation des terres provençales par des populations intrusives de guerriers et d'agriculteurs de souche méditerranéenne venues par bateaux depuis l'Égypte (ibid., p. 53). Cette théorie, qui obéit à des schémas explicatifs diffusionnistes, s'inscrit dans une étape de la recherche marquée du sceau des travaux de V. G. Childe, dans lesquels les originalités occidentales et les capacités créatrices des groupes indigènes en regard des influx est-méditerranéens étaient minimisées (Childe, 1949; Guilaine, 2003).

\section{RHÔNE-OUVÈZE ET FRAISCHAMP: UNE INFLUENCE LANGUEDOCIENNE TOUJOURS DÉCISIVE}

À la fin des années 1980, l'influence des recherches languedociennes s'affirme encore plus dans les propositions qui sont faites sur la genèse des groupes provençaux nouvellement créés: le Rhône-Ouvèze et le Fraischamp (fig. 8).

En effet, si le Rhône-Ouvèze répond dans un premier temps au besoin de faire reconnaître en Provence une manifestation culturelle originale pour la première moitié du $\mathrm{III}^{\mathrm{e}}$ millénaire av. J.-C., c'est en référence aux groupes languedociens que les auteurs caractérisent ce nouveau groupe dans lequel ils trouvent tous les éléments à l'origine de sa formation (Müller et al., 1986, p. 480). Il y aurait ainsi dans le Rhône-Ouvèze «une tradition décorative Ferrières associée à des formes fontbuxiennes" (ibid., p. 480). Les attributs céramiques retenus dans sa détermination, comme les grands contenants à ouverture rétrécie et à cordons souvent en arceaux, les préhensions à ensellement médian, les incisions en guirlande et le 


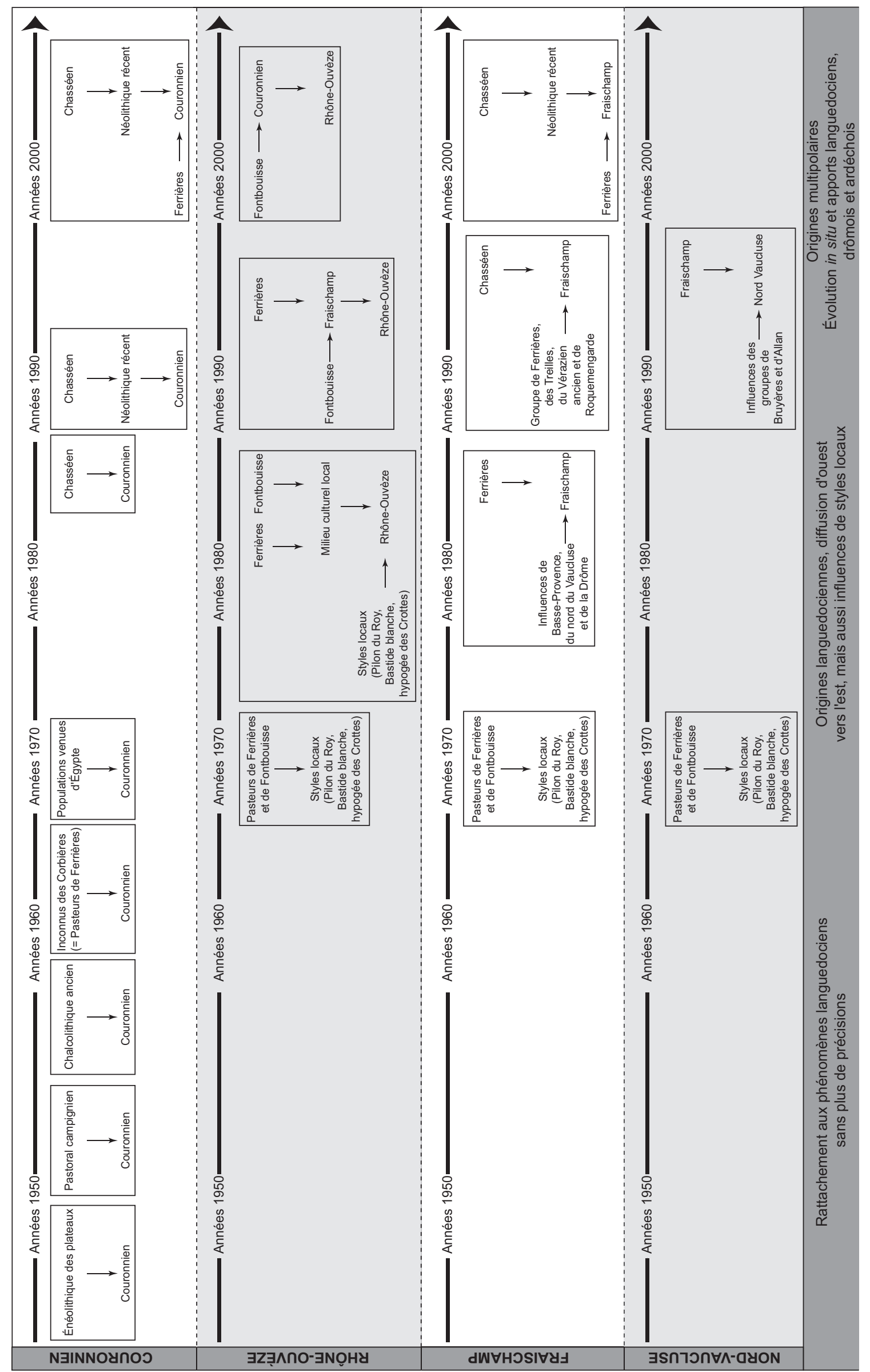

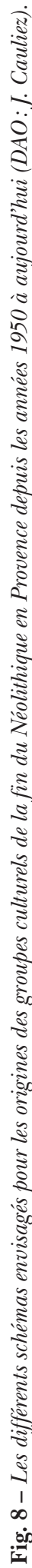

0
0
0
0
0
0
0
0
0
0
0
0
0
0
0
0
0
0 
pastillage, se révèlent par exemple indicateurs de phénomènes de continuité et d'apparentement avec la culture de Ferrières. Parallèlement, la large répartition du Fontbouisse en Languedoc, qui correspond à celle que connaissait antérieurement le Ferrières, se traduit même par une expansion importante vers le nord, par la vallée du Rhône et en Provence intérieure, où de nombreuses séries montrent un phénomène de diffusion permettant, selon les auteurs, de pister les origines du Rhône-Ouvèze (ibid., p. 480).

Quatre années plus tard, dans la première définition du Fraischamp, G. Sauzade remarque lui aussi que la céramique de cet ensemble culturel peut emprunter des éléments décoratifs languedociens, en particulier ceux du Ferrières. L'adoption et l'assimilation de ces influences Ferrières aboutissent à des formes céramiques et à des thèmes décoratifs singuliers qui permettent de caractériser ce nouveau groupe. Il s'agit notamment d'une composition alliant des chevrons à des lignes continues, de motifs de chevrons simples ou imbriqués ou de chevrons simples en pointillés. La combinaison de plusieurs techniques décoratives est aussi rapportée au Ferrières, tout comme les décors plastiques de cordons continus lisses, uniques ou doubles (Sauzade et al., 1990, p. 175).

Ce rapport au Languedoc est directement lié aux avancées que connaît ce secteur géographique au milieu des années 1980. Du côté ouest du couloir rhodanien, le cadre chronoculturel ne cesse en effet de s'affiner grâce à l'étude de nombreux gisements stratifiés et à la réalisation de plusieurs datations radiocarbone. Des évolutions culturelles sont désormais perceptibles dans le temps. Laccent est mis sur la complexité du Néolithique méridional et sur la succession spatiale et temporelle des ensembles (Guilaine, 1986, p. 8). Du côté est en revanche, la multiplicité des styles céramiques recensés quinze ans auparavant par J. Courtin, le manque de fouilles exhaustives et, par là même, de corpus cohérents et datables, rendent particulièrement malaisée la compréhension des phénomènes observés. Avant que les groupes Rhône-Ouvèze, Fraischamp ou Nord-Vaucluse ne soient créés, J. Courtin, dans sa thèse de doctorat (Courtin, 1974), propose pour la Provence, plutôt que des groupes ou des cultures, des styles à faible dispersion géographique, précisés au colloque de Narbonne de 1970 sur Les Civilisations néolithiques du midi de la France (Courtin, 1970; Guilaine, 1970). À côté du Couronnien de Basse-Provence présent au Collet-Redon (Martigues, Bouches-du-Rhône), trois styles locaux sont définis pour le reste de la région et pour la fin du Néolithique, période désignée alors sous le terme de «Chalcolithique provençal»: ceux identifiés sur la station du Pilon du Roy à Allauch dans les Bouches-du-Rhône (Courtin, 1960 et 1977), de la Bastide Blanche à Peyrollesen-Provence dans les Bouches-du-Rhône (Gagnière, 1961) et de l'hypogée des Crottes à Roaix dans le Vaucluse (Courtin, 1976). Pour ces styles, la question des origines est abordée en privilégiant la piste d'un transfert d'influences languedociennes d'ouest en est. J. Courtin affirme notamment, comme l'avait fait auparavant J. Audibert (1962), que la fin du Néolithique de Provence fait figure d'épiphénomène et que cette région ne peut avoir qu'un développement parallèle et secondaire au Languedoc, représentant «un faciès marginal et appauvri des civilisations languedociennes contemporaines, avec lesquelles [la Provence] entretient pourtant des relations étroites» (Courtin, 1974, p. 219). Dans la basse vallée du Rhône, il mettra d'ailleurs en évidence une «colonisation» du groupe de Fontbouisse sur les sites de la place du Palais et de la rue Ferruce à Avignon (Courtin, 1974; Escalon de Fonton, 1974).

Lorsque les premiers groupes sont identifiés, les recherches sur leurs origines locales ne peuvent dès lors que s'appuyer sur des comparaisons avec ces divers styles céramiques régionaux, voire microrégionaux, dont le calage demeure incertain. Il s'ensuit que pour la formation du Rhône-Ouvèze et du Fraischamp, les inventeurs évoquent, en sus des ascendances fontbuxiennes ou Ferrières, celles beaucoup plus imprécises de styles céramiques de BasseProvence de l'ouest (style du Pilon du Roy et de la Bastide Blanche), du nord du Vaucluse (style de l'hypogée des Crottes) ou de la Drôme (Müller et al., 1986, p. 480; Sauzade et al., 1990, p. 176). Pour le Fraischamp, en même temps que G. Sauzade signale l'importance des convergences avec le groupe de Ferrières, ses datations et son extension lui permettent également d'entrevoir son articulation chronologique et géographique avec le Couronnien. Pour chaque groupe donc, des parentés avec les productions indigènes commencent à être mises en évidence. Elles témoigneraient d'un milieu culturel local lui-même en cours de formation et d'évolution, mais qui assimile et intègre de manière invariable les éléments du Languedoc en les régionalisant.

\section{DES ÉVOLUTIONS IN SITU ET UNE NOUVELLE RÉSONANCE LANGUEDOCIENNE}

Progressivement les archéologues tempèrent l'idée d'un transfert total du Languedoc vers la Provence pour explorer cette piste d'une évolution sur place (fig. 8). Ce faisant, les recherches provençales reflètent une tendance assez générale de la Préhistoire récente française (Guilaine, Gutherz, 
1990). En effet, au début des années 1990, les études typologiques de séries mieux documentées, la contribution des longues séquences stratigraphiques dilatées sur des sites à occupation continue ou cyclique et l'étude d'ensembles isolés mais riches et homogènes font reculer toutes les hypothèses diffusionnistes des trente dernières années. Ces longues séquences stratigraphiques permettent d'entrevoir des horizons de transition: on envisage que les ensembles de la fin du Néolithique ne sont pas systématiquement intrusifs et peuvent être issus d'une évolution in situ des groupes locaux du Néolithique moyen ou du Néolithique final. Pour autant, même dans ces nouvelles propositions, l'ascendance du Languedoc sur la Provence demeure toujours aussi prégnante. Les différentes pistes formulées cette dernière décennie sur la question de l'origine des groupes traduisent ainsi toute la complexité et l'interpénétration des phénomènes culturels étudiés. Entre influx exogènes et émergence locale, les origines des groupes sont conçues comme le fruit d'apports «multipolaires» (D’Anna, 1995b, p. 273).

\section{Le Couronnien}

Pour l'origine du Couronnien, l'hypothèse d'une évolution depuis le Chasséen provençal est formulée dans la synthèse de A. D’Anna (1995b). Toutefois, un hiatus entre les dates de ce dernier - de 4500-4300 à 3700 - et celles du Couronnien - de 3100 à 2400 -, ne permet pas de tisser directement des liens entre ces deux ensembles, «à moins que le [Chasséen] ne dure plus longtemps dans le sud-est comme cela a été proposé (Courtin, 1974; Sauzade, 1983; Beeching et al., 1995) ou qu'un autre ensemble ne s'intercale entre les deux»(D'Anna, 1995b, p. 271). Dans l'éventualité de l'existence d'une longue période de transition entre le Néolithique moyen et le Néolithique final, le Couronnien n'apparaîtrait qu'à un moment où le Chasséen stricto sensu aurait disparu depuis un certain temps, remplacé par des groupes évoluant progressivement d'un faciès à un autre. La création d'une entité intermédiaire, le Néolithique récent, définie dans un premier temps en Languedoc sur les sites de l'Avencas à Brissac dans l'Hérault (Gutherz, 1980a) et de la Mort des Ânes à Villeneuve-lès-Maguelone (Gasco, 1980) et reconnue en Provence à la grotte Goulard à Ménerbes (Sauzade, 1990b; Jacob et al., 1990), permet d'évacuer la question du passage du Chasséen au Couronnien. Cependant, le Néolithique récent n'est pas aujourd'hui vraiment documenté; hormis les quelques sites phares du Languedoc, les jalons sont rares en Provence: aucune fouille ne vient confirmer l'existence de cette interface chronoculturelle et des stratigraphies révélant la transition de l'un à l'autre font défaut. Les chercheurs avancent seulement que le Néolithique récent représenterait, comme en Languedoc, une phase ancienne du Néolithique final jouant un rôle dans la formation des groupes, tel le Couronnien (D’Anna, 1995b, p. 273). Si depuis cette proposition, deux nouvelles séries céramiques provençales - la grotte du Mourre de la Barque à Jouques dans les Bouches-du-Rhône et les Ribauds à Mondragon dans le Vaucluse - sont venues alimenter le corpus de sites rattachables au Néolithique récent, les problèmes de l'origine du Couronnien se posent à l'identique (Lemercier, à paraître; Lemercier, Bailly, à paraître; Margarit, à paraître; Van Willigen, à paraître). Ce groupe pourrait se développer dans le dernier tiers du $\mathrm{IV}^{\mathrm{e}}$ millénaire sans lien aucun avec le Chasséen et dans une ambiance appartenant déjà au Néolithique final dans laquelle les traditions chasséennes ne sont plus du tout perceptibles. En Provence, dans la période 3500-3300 av. J.-C., il existerait dans ce cas un ou des groupes proprement attribuables au Néolithique récent, qui restent à reconnaître et à définir précisément et dans lesquels le Couronnien trouverait sa genèse (Lemercier et al., 2003).

D'autres indices révèlent par ailleurs une ambiance similaire entre les groupes de part et d'autre du Rhône, qui repose avec plus d'acuité la question de la place des cultures languedociennes dans la naissance du Couronnien. En effet, le développement des monuments mégalithiques en Provence au début du Néolithique final est un argument supplémentaire pour mettre en relation, toujours selon A. D'Anna, les cultures contemporaines de la diffusion des dolmens: le Ferrières en Languedoc oriental et le Couronnien en Provence occidentale, auquel est attribué notamment le dolmen de Cudières à Jouques (Sauzade, 1988 et 1990a). L'aire géographique d'extension du Couronnien correspond sensiblement à celle des dolmens à chambre allongée (D’Anna, 1999, p. 154). L'extension spatiale du groupe de Ferrières doit encore être précisée, mais sa présence en Provence rhodanienne, par exemple aux hypogées de Fontvieille dans les Bouches-du-Rhône, serait également notable (Sauzade, 1990a; D’Anna, 1995b, p. 279). Ces derniers sont d'ailleurs rattachés dans la littérature, tantôt au Couronnien, tantôt au Ferrières. Dans le même temps, sans savoir encore s'il s'agit d'influences ou de contacts et d'échanges, le Ferrières pourrait être attesté directement sur des établissements provençaux couronniens jusque dans la moyenne vallée de la Durance comme sur le gisement de la Fare à Forcalquier dans les Alpes-de-Haute-Provence (Lemercier, Furestier et al., 2004a). 


\section{Le Fraischamp}

La question des origines du Fraischamp reste également relativement floue. Plusieurs éléments pourraient étayer à nouveau la piste d'une «origine multipolaire » (D’Anna, 1995b, p. 273). Les dates radiocarbone obtenues à la Clairière permettent de situer ce groupe entre 3300 et 2900 av. J.-C., ce qui lui confère une position un peu plus ancienne que le Couronnien. À titre d'hypothèse, il a été proposé que le Néolithique récent pourrait représenter une phase ancienne jouant un rôle dans le Fraischamp, tout comme dans le Couronnien (ibid.). La position stratigraphique des céramiques de type Fraischamp dans les grottes du Luberon occidental, dans un niveau situé immédiatement au-dessus du Chasséen, n'oblitère pas non plus l'éventualité que les origines du groupe soient à rechercher dans un Chasséen finissant. Les caractéristiques techniques de la céramique, fine, bien cuite et polie, ainsi qu'une partie du répertoire des formes et décoratif corroboreraient cette hypothèse: la technique de la gravure et la nature des décors en épis, les formes galbées à paroi convergente, étroites, profondes et hautes ou composites. À cela s'ajoute l'utilisation préférentielle des roches éclogitiques pour la fabrication des lames de haches polies et des éléments de parures similaires au Chasséen. Cependant, le groupe pourrait découler, là encore, de fortes influences languedociennes. Quelques aspects dans les motifs introduisent l'idée d'un apport du groupe de Ferrières naissant, mais le Fraischamp peut être aussi mis en parallèle avec le Vérazien ancien, les premières phases du groupe des Treilles et le groupe de Roquemengarde-Broum. Chacun de ces ensembles languedociens dispose de datations centrées sur la fin du IV millénaire et ils ont tous pu essaimer en Provence (Vaquer, 1990 et 1998).

\section{Le Rhône-Ouvèze}

À propos de la formation du Rhône-Ouvèze, les chercheurs envisagent également plusieurs pistes alliant des influx extérieurs à une mise en place à partir de bases locales. Tout d'abord, une seconde «vague d'influences languedociennes» Fontbouisse, après le passage du Ferrières, a pu interférer dans la genèse du groupe; c'est du moins ce que traduiraient les décors et certaines morphologies céramiques, comme les vases carénés (D’Anna, 1995b, p. 276). Dans la périodisation de A. D’Anna, les datations disponibles situent le Rhône-Ouvèze entre 2900 et 2400 av. J.-C. Il est donc plus récent que le groupe de Fraischamp et contemporain du Couronnien. Étant donné que le Rhône-Ouvèze occupe la même aire géographique que le Fraischamp, il est possible de considérer que le rayonnement du Fontbouisse depuis la rive droite du Rhône se serait diffusé sur un substrat Fraischamp engendrant l'apparition du Rhône-Ouvèze. Il y aurait donc filiation entre ces derniers ensembles culturels, laquelle serait par ailleurs attestée par les formes céramiques et les industries osseuses et lithiques, parfois identiques (ibid., p. 274). Dans un tel schéma, une autre hypothèse, inhérente au fait que le Fraischamp soit lié au Ferrières, a été émise. Elle propose de concevoir que le Rhône-Ouvèze se développe à partir des trois interactions Ferrières-Fraischamp-Fontbouisse (ibid., p. 276).

Une lecture alternative a été avancée depuis peu (Lemercier, 2001). Elle s'appuie sur la mise en évidence d'un fonds céramique commun au Couronnien et au Rhône-Ouvèze (Cauliez, 2001a et 2001b), sur le postulat que l'influence fontbuxienne en Provence est bien perceptible (Cauliez, 2002 et 2007) et enfin sur une réévaluation de la chronologie du Couronnien. Une nouvelle calibration et la correction des datations disponibles pour ce dernier groupe l'inscriraient dans un intervalle plus court que celui proposé par A. D’Anna dans sa périodisation: de 3100 à 2700-2600 av. J.-C. Cela permet alors d'envisager comme quatrième scénario la disparition du Couronnien sous l'impulsion du groupe de Fontbouisse et l'apparition du Rhône-Ouvèze tardivement, peu avant le milieu du III ${ }^{\mathrm{e}}$ millénaire av. J.-C., sur un substrat local couronnien.

\section{Le Nord-Vaucluse}

Pour le quatrième groupe, le site éponyme du Fraischamp à la Clairière livrerait une couche sus-jacente assimilable au Nord-Vaucluse. Cette continuité stratigraphique observée sur le site de la Clairière entre les niveaux du Fraischamp et du Nord-Vaucluse montrerait, d'après A. D'Anna, que ce dernier ensemble culturel pourrait, comme le RhôneOuvèze, résulter d'une évolution du groupe de Fraischamp. En revanche, les influences languedociennes disparaîtraient (D’Anna, 1995b, p. 276). Pour cette entité, toujours d'après A. D'Anna, la question des origines peut être abordée simultanément en rapport avec d'autres entités culturelles, telles que les Bruyères à Saint-Julien-de-Peyrolas dans le Var ou Allan dans la Drôme (Gilles, 1975; Beeching, 1980 et 1986; Gasco, 1990-1991 ; Montjardin, 1990-1991 ; Bordreuil, 1995 et 1998). Ces deux groupes, localisés immédiatement au nord et à l'ouest de ce Nord-Vaucluse, pourraient avoir tenu un rôle décisif dans sa formation (D’Anna, 1995b, p. 276). 


\section{DES TRAMES ET DES ÉVOLUTIONS PARALLÈLES ENTRE LE LANGUEDOC ET LA PROVENCE ?}

Le postulat actuel, bâti sur plus de 60 ans de recherches liées aux travaux réalisés à l'ouest du couloir rhodanien, considère l'hypothèse d'une évolution parallèle des phénomènes culturels observés entre les deux régions pour la fin du Néolithique. Une comparaison avec la trame établie pour la rive droite du Rhône illustre de façon particulièrement démonstrative les similarités dans le rythme d'apparition des groupes, mais aussi dans la chronologie fixée pour les divers ensembles (fig. 5-7). On conçoit aisément après ces observations, couplées à celles faites sur la construction de la fin du Néolithique, que la recherche provençale s'est figée autour de cette clef de voûte que constitue le Languedoc. Cela résulte en partie du fait que les recherches dans cette région ont été historiquement plus avancées grâce à la richesse du contexte archéologique. Les chercheurs des secteurs voisins y ont trouvé une source d'inspiration.

\section{LES GROUPES DE PART ET D'AUTRE DU RHÔNE: RYTHMES D'APPARITION ET CHRONOLOGIE SEMBLABLES}

En Languedoc, la première étape (stade la de A. D'Anna ou stade 0 de $\mathrm{O}$. Lemercier) est commune à une grande partie de la région. Le Néolithique moyen chasséen ne semble plus fournir beaucoup de datations après 37003600 av. J.-C. Dans la période qui suit, entre 3600 et 3100, et peut-être un peu au-delà, des ensembles céramiques mis au jour d'abord en Languedoc (l'Avencas à Brissac, la Mort des Ânes à Villeneuve-lès-Maguelone, l'aven de la Boucle à Corconne dans le Gard), puis en Provence (la grotte Goulard à Ménerbes dans le Vaucluse) et dans la vallée du Rhône (le Duc et les Ribauds à Mondragon) présentent une tradition chasséenne commune où l'apparition d'éléments nouveaux est visible dans les types de décors et certaines morphologies: diminution des ruptures vives de profil, développement des décors plastiques comme les ornements de cordons lisses horizontaux uniques ou multiples, banalité des formes simples, faible variabilité des éléments de préhension et échantillon restreint des décors avec des morphologies simples, agrémentées de cordons ondulés ou en chevrons. L'existence de sites aux fonctions variées (abri, habitat et même site funéraire avec la nécropole de Château Blanc à Ventabren dans les Bouches-du-Rhône) et l'arrêt de la diffusion du silex blond au profit de l'exploitation de silex locaux par percussion (Furestier, 2005) corroborent eux aussi l'hypothèse d'une expression culturelle autonome dans des contextes datés du milieu du IV millénaire av. J.-C. En Languedoc, le développement, à cette date, de l'exploitation des silex bédouliens gris pour la production de petites lames est un élément supplémentaire à la caractérisation d'un stade de transition; c'est du moins ce que suggère la série du site de la Grange des Merveilles à Rochefort-duGard (Monnet et al., 2002). Des décalages chronologiques d'une région à l'autre et l'apparition de groupes régionaux distincts sont probables, mais ils ne sont pas encore clairement mis en évidence. Ces groupes et la période, qualifiés de Néolithique récent, seraient synchrones avec les cultures plus septentrionales (Horgen, Seine-Oise-Marne).

La deuxième étape (stade $1 \mathrm{~b}$ de $\mathrm{A}$. D'Anna ou stade $1 \mathrm{de}$ O. Lemercier) correspond à la mise en place et à l'évolution des cultures matérielles du Néolithique final qui semblent s'effectuer principalement de 3500-3400 à 2900 av. J.-C., pour le groupe de Ferrières en Languedoc oriental et les ensembles Fraischamp puis Couronnien en Provence.

La troisième étape (stade 2a de A. D'Anna ou stade 2 de O. Lemercier) est marquée par le développement du groupe de Fontbouisse et de ses faciès géographiques dans un intervalle s'étendant de 2900-2800 à 2600-2500 av. J.-C., pendant lequel la Provence est occupée par le groupe couronnien. La large répartition du groupe de Fontbouisse correspond à celle que connaissait le groupe de Ferrières antérieurement et présente même une expansion importante vers le nord, par la vallée du Rhône et en Provence intérieure où de nombreuses séries montrent un phénomène de «mixage stylistique». L'entité culturelle Rhône-Ouvèze, contemporaine du groupe de Fontbouisse, résulterait de ce processus. Parallèlement, le groupe du Nord-Vaucluse serait constitué par d'autres types d'influence.

La quatrième étape de la périodisation (stade $2 \mathrm{~b}$ de A. D’Anna ou stades 3 et 4 de O. Lemercier) correspond à l'apparition et au développement du Campaniforme. En Languedoc, sa présence est attestée dès 2500 av. J.-C. dans de rares ensembles culturels Fontbouisse. En Provence, le Campaniforme s'intègre dans le Rhône-Ouvèze à des dates similaires.

Dans un tel schéma, on aurait alors une coexistence, dans les derniers siècles du IV e millénaire du Ferrières, du Fraischamp et du Couronnien, puis, entre 2900 et 2500, du Couronnien, du Fontbouisse, du Nord-Vaucluse et du Rhône-Ouvèze (fig. 5 et 6 ). Des rapports entre les groupes sont aussi identifiés. Le Ferrières comme le Fontbouisse auraient largement diffusé les produits céramiques en dehors de leur périmètre de définition. Ils semblent présents 
en rive gauche du Rhône dans le Vaucluse, avec quelques vases caractéristiques sur les sites d'Avignon (Courtin, 1974; Escalon de Fonton, 1974). Ces deux groupes étendent par ailleurs leur influence jusqu'en Provence occidentale ou en Haute-Provence. Enfin, des faciès périphériques (appelés aussi rhodaniens) ont été délimités dans le secteur de la vallée du Rhône, où des phénomènes de transition et de frontières avec les complexes culturels provençaux seraient perceptibles (Gutherz, Jallot, 1995; Jallot, 2003). De là découlent naturellement de multiples possibilités d'interaction entre les ensembles culturels locaux, ce dont témoignent les nombreuses propositions énoncées sur l'origine des groupes provençaux.

Dans l'hypothèse d'une évolution parallèle des deux pôles géographiques, il ressort de ces quelques observations, outre le grand dynamisme culturel de la région à la fin du Néolithique, que le milieu culturel local est perméable aux influx languedociens. Le Languedoc constituerait un secteur de développements culturels importants et successifs et la Provence, un secteur récepteur systématique de ces développements. Ce sont les entités d'origine languedocienne qui semblent avoir marqué le plus profondément la région et constitué, à l'inverse, des ensembles très peu réceptifs aux influences extérieures.

\section{LES LIMITES D'UNE LECTURE BINAIRE LANGUEDOC/PROVENCE}

Qu'en est-il aujourd'hui de la pertinence des seuls parallèles Languedoc/Provence dans les schémas de formation des groupes ? Le rythme d'apparition des divers ensembles est-il réellement similaire de part et d'autre du Rhône? Pouvons-nous apprécier autrement la valeur culturelle de la sphère provençale et quelle validité donner aux hypothèses de formation des groupes in situ?

En Languedoc, la qualité et la quantité des données disponibles ont généré l'élaboration d'une succession chronologique moins complexe et mieux établie qu'en Provence, où des écarts importants sont perceptibles dans de nombreux aspects de la périodisation. Quatre points principaux dans les hypothèses formulées sur la mise en place des groupes provençaux méritent, selon nous, d'être révisés et montrent que les nombreux parallèles énoncés avec le Languedoc ne permettent pas de répondre à toutes les questions.

Tout d'abord, le Néolithique récent demeure mieux connu en Languedoc depuis trente ans, alors que la documentation sur cet intervalle culturel est encore très lacunaire de l'autre côté du Rhône. Le travail de définition, qui reste d'ailleurs aussi à faire en Languedoc, est important pour déterminer les liens entre ce Néolithique récent et la mise en place des groupes du Néolithique final provençal. Le but est de savoir si ces derniers dépendent plus de modalités locales d'adaptation que d'un véritable essaimage de cultures depuis la rive droite du Rhône.

Au-delà de cet aspect, s'il existe une évolution parallèle des deux régions, il semble en revanche que la Provence soit la seule dans une situation de dépendance par rapport au Languedoc, tout du moins pour ce qui a trait aux productions céramiques. Plusieurs travaux ont mis en exergue toute l'importance de la formation des groupes sur la base d'une évolution et d'une transformation du milieu culturel local, mais c'est toujours au regard d'influx occidentaux que ces changements s'opéreraient. Cette vision ne pourrait-elle pas masquer les originalités provençales, les capacités créatrices des groupes autochtones et toutes leurs diversités, voire même l'apport d'autres influx du nord-est ou de l'est, par exemple, dans leur formation?

Plusieurs constats vont d'ailleurs dans ce sens. Les distributions géographiques actuelles des ensembles provençaux ne corroborent pas systématiquement la piste d'une filiation entre eux. Ils ne reprennent pas strictement la même répartition que le groupe précédent, comme cela semble être le cas entre le Ferrières et le Fontbouisse. Souvent, les entités prennent forme ailleurs, restent limitées à un secteur ou alors s'étendent largement. De plus, même si de nombreux éléments de formes et de décors sont similaires entre les cultures de la rive gauche du Rhône, celles-ci ne paraissent pas proposer, à l'image du Ferrières et du Fontbouisse, une homogénéité typologique et stylistique particulièrement stable sur plusieurs siècles. En termes de succession, le passage du Ferrières au Fontbouisse est défini en Languedoc de manière précise sur plusieurs sites livrant des indices stratigraphiques d'une filiation (Jacobieski, 1988; Gutherz, Jallot, 1995, p. 242; Jallot, 2003, p. 246), tandis qu'en Provence, les connections au sein des groupes ne sont traitées que sous l'aspect de ressemblances ou de divergences typologiques, sans la validation du cheminement d'un ensemble culturel à l'autre. Les gisements stratifiés susceptibles de fournir des séquences longues révélant des horizons de transition, par exemple du Fraischamp au Couronnien ou du Couronnien au RhôneOuvèze, font défaut. Sur la question des origines locales, la Provence peut donc connaître elle aussi, dans certaines zones et à certains moments de la chronologie, des développements originaux et parfois durables qu'il reste à définir, 
indépendamment des a priori dus au poids des interactions ouest-rhodaniennes.

D'autre part, si la diffusion de spécificités languedociennes sur la région est clairement démontrée, elle mérite là aussi d'être précisée, d'autant que les données témoignent que les groupes du côté ouest du Rhône pourraient influer de manière différente en fonction du substrat local provençal. Bien que le Ferrières et le Fontbouisse semblent interférer sur les phénomènes de la rive gauche et ont un rôle important sur l'ensemble de la région, il apparaît dans les différentes hypothèses formulées que cette ascendance s'opère selon une intensité différentielle en fonction du milieu culturel autochtone: le Fraischamp serait particulièrement concerné par une influence Ferrières, alors que le Couronnien, pourtant en partie synchrone, le serait beaucoup moins. De la même manière, le groupe RhôneOuvèze illustrerait le poids d'interactions fortes avec le Fontbouisse, voire même avec le Ferrières, tandis que le groupe Nord-Vaucluse, contemporain du Rhône-Ouvèze, ne serait pas touché par de tels apports. Dans ces propositions, on constate aussi deux modes de diffusion de l'ouest vers l'est. Soit l'influence des groupes du Languedoc sur les ensembles provençaux s'opère de façon immédiate: le Fraischamp viendrait du Ferrières, tout comme le RhôneOuvèze viendrait du Fontbouisse. Soit, elle transite par plusieurs intermédiaires et l'emprise est alors moins forte dans la constitution du groupe. C'est le cas du Couronnien, qui porterait en lui l'empreinte Ferrières, qui aurait d'abord voyagé à travers le Fraischamp. Dernière interrogation sur ce point: si les influences du Languedoc oriental au cours du Néolithique final paraissent constantes et récurrentes dans la céramique provençale depuis 3400 av. J.-C., de quels phénomènes s'agit-il: imitation, transfert stylistique ou migration des groupes Ferrières et Fontbouisse vers les groupes Fraischamp et Rhône-Ouvèze de la rive gauche (Giligny, 1993 et 1997)?

Par ailleurs, dans toutes les hypothèses proposées ces dernières décennies sur la formation des groupes, le Nord-Vaucluse serait le seul non concerné par des influx languedociens et sans doute le résultat du fruit d'interactions avec des entités culturelles placées plus au nord de la région. Ne faut-il pas alors tenter de comprendre la structuration des groupes provençaux en examinant la possibilité d'ascendances autres que languedociennes ? Aujourd'hui, ces dernières pourraient donner de nouveaux points d'ancrage chronologiques et géographiques aux groupes locaux de la fin du Néolithique provençal et expliquer que nos ensembles culturels à l'est du Rhône s'insèrent dans un phénomène de microrégionalisation et apparaissent variés dans leurs manifestations.

En quelques mots, il est important aujourd'hui de se fixer de nouveaux points de repère, de reconstruire ou de réactualiser le système global de référence, de rediscuter les interprétations proposées sur la structuration chronoculturelle du Néolithique final en y intégrant de nouvelles données, pour ensuite seulement tenter de confronter et d'homogénéiser nos cadres avec les divers grilles chronotypologiques du Midi méditerranéen (Voruz, 1995, p. 14).

\section{LA QUESTION DE L'ÉVOLUTION ET DE LA TRANSFORMATION DES GROUPES DURANT LA FIN DU NÉOLITHIQUE EN PROVENCE}

Pour le sud de la France, malgré les réseaux complexes et foisonnants régissant les sociétés, les groupes culturels sont encore définis comme des civilisations monothétiques, dans le sens de D.-L. Clarke (1978, p. 30-41), c'est-à-dire comme des blocs culturels voisins et juxtaposés et dont tous les éléments de la culture matérielle seraient stables ou varieraient ensemble dans le même sens (Pétrequin et al., 1987-1988). Cette notion, jugée longtemps commode, résiste de plus en plus mal aux données actuelles qui montrent des processus complexes d'interaction entre les cultures, aboutissant tantôt à la genèse de nouveaux groupes, tantôt à leur disparition. Il ne s'agit donc pas simplement d'une opposition ou d'une succession groupe par groupe. Il y a très certainement des espaces culturels communs, des rythmes et des modes de peuplement divers. Ici, l'ambition est de rappeler, pour chacune des cultures néolithiques du Midi méditerranéen, les mécanismes de leurs imbrications, leur variabilité dans l'espace et dans le temps, leur spécificités locales ou alors leur capacités à s'intégrer à d'autres groupes, afin de mieux comprendre en quoi elles sont interactives et complémentaires, indépendantes ou autonomes.

\section{MUTATIONS ET INTERACTIONS EN PROVENCE: LES HYPOTHÈSES ACTUELLES}

Les hypothèses formulées concernent les différents changements géographiques, chronologiques ou culturels connus pour chaque groupe en rive est du Rhône. Le Fraischamp n'est pas traité, car, en dehors des données sorties du site éponyme, l'habitat de la Clairière, on connaît relativement peu de choses sur ses évolutions possibles. Le 


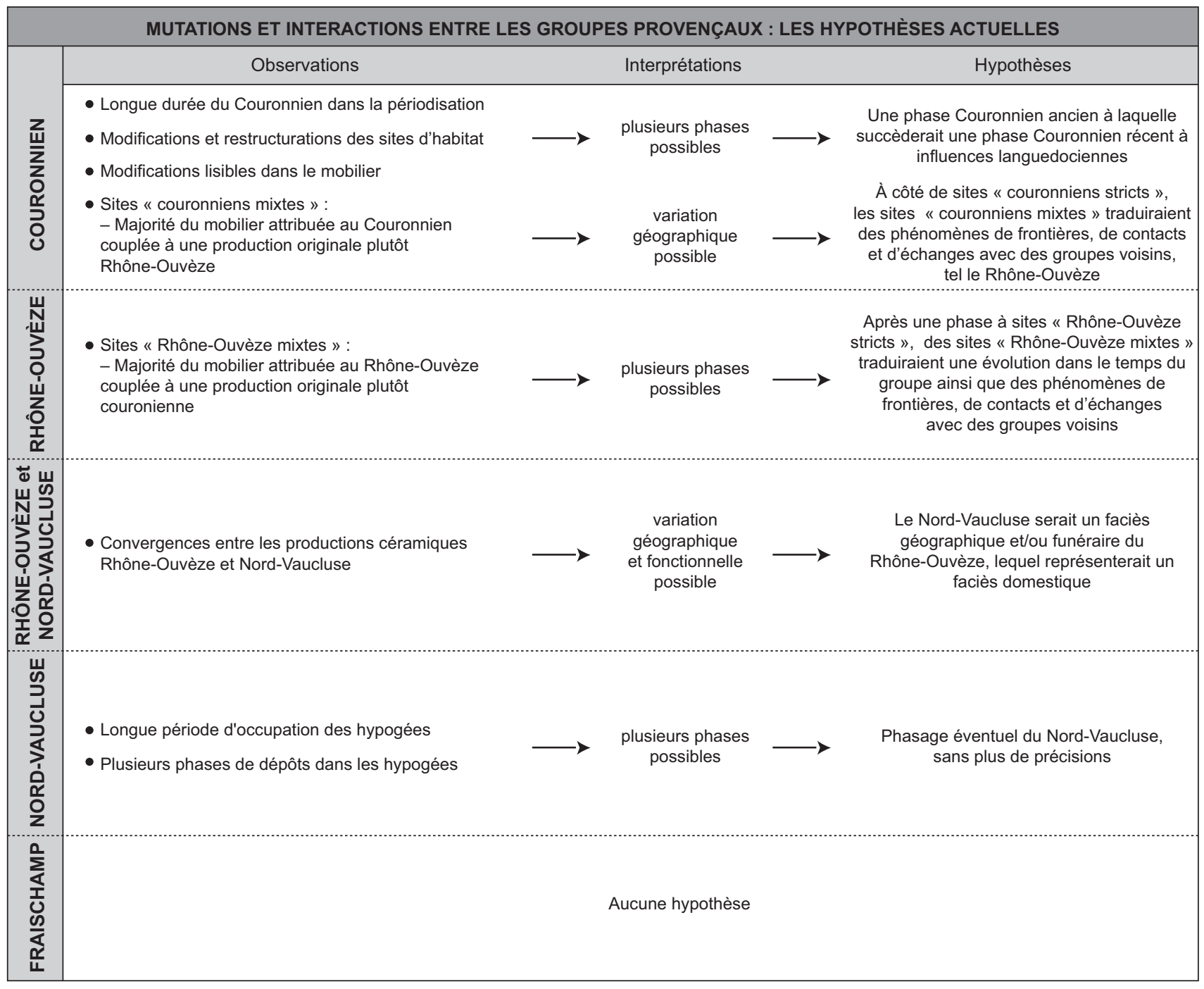

Fig. 9 - Les différents schémas envisagés actuellement pour les mutations et les interactions des groupes culturels de la fin du Néolithique en Provence (DAO: J. Cauliez).

groupe couronnien est abordé à part. Quant au RhôneOuvèze et au Nord-Vaucluse, ils sont examinés ensemble, car leur transformation est en partie liée.

\section{LE COURONNIEN : \\ PHASAGE ET VARIATION GÉOGRAPHIQUE}

En termes d'évolutions chronologiques, les données sur le Couronnien sont lacunaires. Dans la périodisation de A. D’Anna, les datations ${ }^{14} \mathrm{C}$ réalisées sur plusieurs sites attribués au groupe couvrent une période relativement étendue de 900 ans, de 3300 à 2400 av. J.-C. Cette durée serait confirmée par les indices d'occupation longue des établissements: des phénomènes de transformation sont visibles en particulier dans l'architecture. Par exemple, au Collet-Redon, à la Couronne ou à Ponteau-Gare à Martigues dans les Bouches-du-Rhône, les aménagements ont fait l'objet de restructurations et de modifications (Gilabert, 2002; Durrenmath et al., à paraître). À Miouvin (Istres, Bouches-du-Rhône), aux Lauzières (Lourmarin), aux Martins (Roussillon), comme aux Fabrys (Bonnieux), les datations et les recoupements/recouvrements de l'habitat suggèrent également une longue occupation des sites où le passage d'un habitat ouvert à un habitat partiellement enclos par une enceinte est particulièrement identifié (Bretagne, D’Anna, 1988; D'Anna et al., 1989; D’Anna, 1989 
et 1999, p. 154; Lemercier, Gilabert, 2009). Le réexamen global engagé sur les collections céramiques indique également que des phénomènes de transformation se lisent dans le mobilier: si les premières études évoquent un large fonds attribuable au Couronnien, les séries de référence montrent des ensembles qui ne sont pas totalement homogènes (Cauliez, à paraître b). Des évolutions internes au groupe seraient donc possibles (fig. 9).

Les hypothèses établies pour chacun de ces sites sont celles de l'existence de plusieurs phases principales dans l'histoire des établissements. Une phase ancienne se rattacherait au Couronnien stricto sensu et une autre, ultérieure, pourrait correspondre à un Couronnien à influences languedociennes. Les Lauzières (Delaunay, 2001), PonteauGare (Margarit, 2002) ou le Collet-Redon (Cauliez, à paraître b) semblent en effet intégrer dans un second temps du mobilier céramique original. Précisons à ce sujet que l'idée d'une phase récente au Couronnien a été émise très tôt, notamment dans les travaux de J. Courtin. Le style identifié sur le site du Pilon du Roy (Allauch, Bouches-duRhône) était considéré comme caractéristique d'un stade avancé dans l'évolution du groupe (Courtin, 1977).

A. D'Anna a introduit, à la suite de ces différentes observations et en s'appuyant sur une cartographie des gisements couronniens, la possibilité qu'en plus d'une évolution chronologique, le Couronnien puisse connaître des variations géographiques (fig. 9). Il en retient le concept de sites «couronniens mixtes» (D’Anna, 1995b, p. 274). Il pourrait exister, en particulier dans le massif des Alpilles, autour de celui-ci, et dans la région du confluent du Rhône et de la Durance, une zone de contact entre le Couronnien et le Rhône-Ouvèze. Ainsi, à côté de sites «couronniens stricts», plusieurs gisements, dont la plus grande partie du mobilier suggère bien un rattachement à cette culture, présenteraient une série plus ou moins importante d'objets faisant figure d'éléments extérieurs; c'est par exemple le cas aux Lauzières et aux Martins dans le Vaucluse. La présence de pièces non conformes à la définition stricte est alors expliquée par la contemporanéité et les contacts avec les groupes voisins sous la forme de phénomènes de frontière et d'échanges dans une phase récente du Couronnien. Pour étayer ces interactions entre ce groupe et les ensembles culturels d'influence Fontbouisse tels que le Rhône-Ouvèze, J. Vaquer va même plus loin. Il propose de voir, en lieu et place du Rhône-Ouvèze, un faciès récent du Couronnien: «Le Couronnien se place globalement entre 3100 et 2400 ; le style Rhône-Ouvèze est contemporain du Fontbouisse (2800-2200) et constitue donc bien un faciès occidental du Couronnien récent» (Vaquer, 1998, p. 457). Ce ne sont cependant que les productions céramiques et les aménagements architecturaux qui livrent les premiers traits discriminants au sein du groupe et dans lesquels de possibles transformations entre les différentes phases d'occupation des sites sont lisibles. En effet, les autres produits manufacturés ont tendance à traduire une homogénéité typologique et technologique remarquable, notamment l'industrie lithique polie ou taillée et la parure (Lazard, 2001; Pellissier, 2001; Furestier, 2002).

Plus récemment, un second schéma dégagé dans la périodisation de O. Lemercier reprend la possibilité de sites "couronniens mixtes», mais dans une chronologie plus restreinte du groupe (Lemercier, 2007). Vingt-quatre datations attribuées au Couronnien centreraient, après calibration, cet ensemble dans la première moitié du III $^{\mathrm{e}}$ millénaire av. J.-C. (Lemercier, 2001). Certaines dates confrontées à l'observation de la trame chronoculturelle régionale montreraient la possibilité de placer l'apparition du groupe couronnien à la fin du IV e millénaire (vers 3000-2900 av. J.-C.). Parallèlement, l'étude récente du Campaniforme dans le sud-est de la France a révélé l'absence d'association ou de relation entre le Campaniforme et le Couronnien, donnant un terminus post quem pour le groupe couronnien vers 2600-2500 av. J.-C. Dans ce cadre alternatif, le processus d'évolution du Couronnien est perçu à l'identique: des interactions chronologiques et géographiques sont reconnues avec le groupe Rhône-Ouvèze, mais également avec le Fontbouisse dans une phase d'évolution particulière du Couronnien placée vers 2800-2700 av. J.-C., sans toutefois pouvoir caractériser plus avant les jeux d'influences réciproques et les modalités de cette transformation.

\section{LE RHÔNE-OUVÈZE ET LE NORD-VAUCLUSE : VARIABILITÉS CHRONOLOGIQUES ET SPATIALES ET FACIÈS}

Dans la périodisation de A. D'Anna, le Rhône-Ouvèze apparaît plus tardivement que le Couronnien, aux alentours de 2800 av. J.-C. (D’Anna, 1995b). Ce groupe connaîtrait lui aussi deux phases successives (fig. 9) : une ancienne attestée par quelques établissements dits "Rhône-Ouvèze mixtes" (ibid., p. 274), comme la Bastide Blanche à Peyrolles-enProvence, Escanin aux Baux-de-Provence ou les Passadouires à Orgon, tous dans les Bouches-du-Rhône, et une autre, ultérieure, Rhône-Ouvèze au sens strict. Les sites associés à la phase ancienne se caractérisent par un mobilier typique du Rhône-Ouvèze combiné à quelques formes simples 
semblant renvoyer au groupe couronnien. Ces sites composites matérialiseraient un temps d'interaction pendant lequel les groupes couronnien et Rhône-Ouvèze seraient en relation de frontières, d'échanges ou de contacts. On voit là que les explications données à ces phénomènes de transformation sont donc sensiblement identiques à celles émises pour les gisements «couronniens mixtes", à ceci près que cette fois-ci le processus est inversé.

Une seconde hypothèse envisage que le Rhône-Ouvèze se décline en faciès géographiques et fonctionnels. À partir de convergences dans la typologie et les styles céramiques du Rhône-Ouvèze et du Nord-Vaucluse, A. D’Anna propose de voir, dans ce dernier, un faciès géographique du RhôneOuvèze circonscrit à la limite nord du Vaucluse (fig. 9). Le caractère sépulcral de l'ensemble des découvertes attribuées au Nord-Vaucluse l'ont incité aussi à envisager ce groupe comme le style funéraire du Rhône-Ouvèze, lequel représenterait la culture domestique identifiée dans le reste de la région sur les sites d'habitat (D’Anna, 1995b).

Enfin, la longue utilisation et les multiples phases de dépôt des hypogées attribués au Nord-Vaucluse laissent supposer que cet ensemble culturel a pu connaître par ailleurs des changements dans le temps. Mais, le faible effectif des gisements associés à ce groupe, sa vocation exclusivement sépulcrale, ainsi que sa délimitation à un petit espace géographique, ne permettent pas de pousser plus avant les interprétations.

\section{FRACTIONNEMENT D'UNE RÉALITÉ HOMOGÈNE OU GLOBALISATION D'UNE RÉALITÉ COMPLEXE ?}

\section{UN PAYSAGE GULTUREL FIGÉ}

Dans le paysage culturel tel qu'il a été envisagé jusqu'à présent, les études sur les diverses entités n'abordent donc que tardivement, en particulier dans les articles de synthèse de A. D'Anna, les problématiques sur les évolutions et les transformations des groupes (D'Anna, 1995a, 1995b et 1999). La difficulté à ordonner les cultures de manière à rendre compte des variations géographiques et chronologiques au sein de ces ensembles considérés comme homogènes résulte en grande partie de l'état d'avancement des recherches: très peu de sites sont exploitables pour de telles approches, lesquelles nécessitent en effet une vision diachronique et élargie à l'ensemble de la région. C'est d'ailleurs dans un cadre chronoculturel peu ou prou figé, dans une aire géographique encore très inégale que les acteurs de la recherche sont encore contraints d'envisager le déploiement et l'évolution des sociétés humaines de la fin du Néolithique. Le déséquilibre est très net: les points d'ancrage chronologiques et géographiques manquent dans les Alpes-Maritimes, le Var et les Alpes-de-Haute-Provence. L'absence significative de datations fiables approfondit ces lacunes. On comprend dès lors que les différentes hypothèses sur la mutation des ensembles envisagent la question de façon relativement succincte.

Cependant, au-delà de ces aspects, les difficultés à cerner les phases d'évolution et de transformation des groupes provençaux peuvent s'expliquer en examinant la question de la dimension géographique donnée à ces cultures. On l'a dit, cette combinaison de quatre entités au III $^{\mathrm{e}}$ millénaire intègre un espace microrégionalisé. Trois des quatre groupes sont reconnus dans le Vaucluse. De plus, le Fraischamp comme le Nord-Vaucluse, restent limités en particulier à ce département. A contrario, le Couronnien et le Rhône-Ouvèze disposent d'une extension plus importante, même si leur élargissement vers l'est dans les Alpes-de-Haute-Provence, le Var et les Alpes-Maritimes reste à préciser.

Face à une telle segmentation, au moins pour le nord de notre zone d'études et au regard d'une telle unité pour le Couronnien et le Rhône-Ouvèze dans le reste de la Provence (fig. 1-4), se repose alors avec plus d'acuité la question de la pertinence de l'échelle à laquelle est défini chacun de ces ensembles. Le fait d'individualiser ou non des groupes à partir de critères parfois trop ténus, de recréer à l'infini des entités ou des cultures standard, se mesurant seulement à l'échelle de la microrégion ou d'un département, a été une des inquiétudes centrales dans l'histoire de la recherche en Préhistoire récente (Bailloud, 1970, p. 91-92). En vis-à-vis, la discipline montre aussi le travers inverse, qui a consisté bien souvent en un élargissement de l'échelle d'analyse par le rassemblement des cultures dans des complexes très vastes lorsque celles-ci avaient entre elles suffisamment de points communs (Courtin, 1970; Guilaine, 1976a et 1980).

Aujourd'hui, nos questionnements et nos craintes sont identiques. Tous les termes du débat tournent autour de la recherche d'un équilibre entre le fractionnement à outrance d'une réalité peut-être homogène et la globalisation à l'excès d'une réalité pouvant être complexe (Bailloud, 1970, p. 91-92). L'enjeu est de décrire et de définir leurs modalités d'évolution et de transformation dans le détail, pour ensuite rattacher les différents groupes chronoculturels entre eux et caractériser leurs processus d'interaction. 


\section{LE FRAISCHAMP ET LE NORD-VAUCLUSE, DES ENSEMBLES TROP CLOISONNÉS ?}

Si l'on s'attarde sur la définition du Fraischamp et du Nord-Vaucluse, il est frappant de constater par exemple, que chacun d'eux semble constituer des particularismes géographiques limités aux monts du Vaucluse et au Luberon, répondant traits pour traits aux critères de définition non pas d'un groupe, mais d'un faciès, tels qu'ils ont été posés dans les travaux languedocien ${ }^{3}$ : des unités typologiques et stylistiques limitées à une aire spatiale et se rapportant à un ensemble plus vaste. Les hypothèses formulées sur l'origine des groupes émettent en effet largement la possibilité que le Fraischamp dépende du Ferrières et que le Nord-Vaucluse constitue un faciès géographique du Rhône-Ouvèze (voir supra, p. 268).

Plus encore, les spécificités fonctionnelles et d'implantation du Fraischamp et du Nord-Vaucluse contribuent à faire de ces deux ensembles des entités isolées. On remarque en effet que les occupations en grottes semblent jouer un rôle primordial dans le Fraischamp; ce dernier étant exclusivement présent dans les grottes du Luberon occidental et dans celle de Saint-Gervais à Bonnieux (D'Anna, 1999, p. 149), tandis que le Nord-Vaucluse est caractérisé systématiquement par des sites funéraires en hypogée.

En Provence, l'accentuation de la diversité des phénomènes culturels de la fin du Néolithique par des ensembles très délimités pose alors une question phare: en quoi les caractères (céramiques notamment) du Fraischamp et du Nord-Vaucluse légitiment-ils la création d'unités culturelles propres au Vaucluse ? Ces deux groupes ne pourraientils pas simplement constituer des points d'ancrage, de transitions et de frontières gravitant autour de complexes

3. J. Arnal a été le premier à envisager avec C. Burnez et J. RoussotLaroque, dans la publication du site de la station du Gravas (Saint-Mathieu-de-Tréviers, Hérault), une méthode d'inventaire et de comptage permettant l'élaboration des premières listes typologiques et la mise en évidence, au sein des cultures languedociennes, de faciès régionaux (Arnal et al., 1967). R. Montjardin a ensuite repris, à la fin des années 1960, une lecture fine de ces ensembles géographiques pour le département de l'Ardèche (Montjardin, 1965 et 1966). Quelques années plus tard, c'est au tour de X. Gutherz de fractionner l'ensemble du Languedoc en faciès céramiques, d'abord pour le groupe de Fontbouisse, puis pour celui de Ferrières. Ces faciès sont déterminés à partir de critères simples de présence/absence ou d'associations préférentielles de caractères morphologiques et/ou ornementaux dans la céramique. Ils sont déclinables d'un groupe à l'autre et se répartissent sur le Languedoc en fonction de secteurs géographiques spécifiques qui correspondent à l'aire d'extension des unités stylistiques et typologiques. Ils ne constituent, par conséquent, que des subdivisions microrégionales ne tenant pas compte de la dimension temporelle (Gutherz, 1990, p. 236). fédérateurs ? (Clarke, 1978, p. 299-327; Pétrequin et al., 1987-1988, p. 2-4).

\section{UNE TROP GRANDE UNITÉ DANS LE COURONNIEN ET LE RHÔNE-OUVÈZE ?}

Il ne faut pas non plus, par excès contraire, globaliser une réalité culturelle qui peut apparaître bien plus complexe. Les groupes couronnien et Rhône-Ouvèze en constituent de parfaits témoignages. En effet, tous deux proposent des extensions géographiques importantes et, en termes de chronologie, ces ensembles s'inscriraient dans un grand intervalle, notamment le Couronnien pour lequel la fourchette temporelle est évaluée à près d'un millénaire. Pour autant, tout en leur reconnaissant des sites mixtes ou l'existence de possibles phasages internes (voir supra, p. 272-273), on présume pour chacun d'eux qu'ils forment des ensembles parfaitement homogènes au sein de ce large espace/temps. D'un point de vue pragmatique, l'augmentation importante du nombre de sites, imputés depuis vingt ans à ces groupes pas encore clairement définis, pose de plus en plus de problèmes. Cela se traduit notamment par le fait que seuls quelques types céramiques emblématiques d'une appartenance culturelle sont pris en compte, au détriment du reste de l'outillage. À côté de sites de référence caractéristiques du groupe, la tendance est à l'attribution souvent rapide d'un gisement ou d'une occupation à une de ces cultures sur la base exclusive d'une portion, parfois minime, du mobilier. Il est aussi récurrent d'assembler un nombre de sites au Couronnien ou au Rhône-Ouvèze, uniquement en raison de leur proximité régionale et chronologique, bien que leur gamme de formes ou de décors céramiques, soit finalement très diversifiée.

Ne pouvons-nous donc pas envisager que le Couronnien ou le Rhône-Ouvèze puissent être davantage compartimentés ? Une fois identifié l'ensemble des éléments par lesquels s'incarne l'unité du groupe, ne peut-on pas apprécier sa diversité, sans pour autant le contraindre par un surfractionnement (Gernigon, 2004) ?

L'appréhension de ces questions passe par le rétablissement d'un va-et-vient entre des échelles d'analyse complémentaires, du micro au macro. Ce faisant, les tendances actuelles marquent cette volonté. Pour rester dans les comparaisons historiques avec le Languedoc, les chercheurs de cette région tentent notamment de définir des séquences locales pouvant conduire aux premiers essais de phasage au sein des deux groupes Ferrières et Fontbouisse. Ils entreprennent pour cela de multiplier les recoupements 
microrégionaux sur des territoires de plus en plus limités (Montjardin, 1988-1989 et 1990-1991; Gutherz, Jallot, 1999). Les relations entre les faciès du Ferrières et du Fontbouisse sont ainsi disséquées, bien au-delà de la seule juxtaposition d'assemblages de mobiliers voisins. Ces nouvelles approches, développées notamment par L. Carozza sur la vallée de l'Hérault (Carozza, 2000 et 2005) et par L. Jallot à l'occasion de la publication du site des Vautes à Saint-Gély-du-Fesc dans l'Hérault (Jallot, 2003), modifient les cadres conceptuels. Elles s'attachent ensuite à mettre en avant les dynamiques des styles céramiques languedociens sur une longue chronologie et, finalement, au sein d'un cadre géographique large où prévalent évolutions et interactions.

Pour traiter ces aspects plus avant, il faut cependant pouvoir relier les différents groupes chronoculturels de la fin du Néolithique provençal à des complexes vastes au-delà de la région, caractériser les processus d'extension ou d'interaction et saisir la perméabilité des communautés néolithiques. Nous proposons donc maintenant d'élargir chronologiquement et géographiquement notre champ de réflexion.

\section{RELATIONS EXTRARÉGIONALES ET MÉCANISMES D’IMBRICATION}

Si nous dépassons les limites régionales et chronologiques du Fraischamp, du Couronnien, du Rhône-Ouvèze et du Nord-Vaucluse, quatre nouveaux aspects dans la transformation des sociétés néolithiques peuvent être abordés.

Tout d'abord, au-delà de leur zone d'extension maximale, les acteurs provençaux peuvent également influer sur leurs voisins culturels. Dans la moyenne vallée du Rhône par exemple, l'existence de mécanismes d'imbrication serait patente entre les cultures provençales et celles des régions du Languedoc, de l'Ardèche et de la Drôme.

Inversement, les phénomènes culturels provençaux ne sont pas systématiquement opératoires dans des schémas de mise en place et d'évolution de groupes exogènes pour lesquels, pourtant, les cultures du sud de la France et en particulier du Languedoc, s'imposent comme des éléments structurants essentiels. Ainsi, la construction des ensembles culturels de l'axe Saône-Rhône est assimilée aux manifestations Ferrières et Fontbouisse et ne serait pas concernée par les influx de la rive est du couloir rhodanien!

Les phénomènes culturels extérieurs ont pu aussi avoir des répercutions fondamentales sur le fonctionnement des entités en place en Provence. Dans le cadre général de l'expansion, au milieu du III ${ }^{\mathrm{e}}$ millénaire, des cultures d'Italie centrale et septentrionale d'abord et du Campaniforme ensuite, la Provence peut être assimilée à une région cristallisant des apports d'origines diverses. En tant que telle, elle enregistre deux types d'informations: d'une part, les conséquences de ces apports extérieurs sur les origines et le développement des groupes locaux et, d'autre part, les évolutions culturelles produites hors de la Provence dans les régions périphériques.

Enfin, au passage vers le Bronze ancien, les cultures locales disparaîtraient peu à peu en rapport avec l'implantation campaniforme. Quelles sont alors les modalités de cette extinction et quel est le rôle des groupes autochtones dans la genèse du Bronze ancien?

Apprécier ces quatre cas de figure constitue un dernier axe de recherche, l'objectif étant de présenter et de coordonner des informations de sphères géoculturelles variées pour se préoccuper de la réinscription du Néolithique final de Provence dans un cadre beaucoup plus global.

\section{LES GROUPES CULTURELS PROVENÇAUX HORS DES LIMITES RÉGIONALES ?}

\section{Languedoc et Ardèche : des espaces potentiellement réceptifs aux influences couronniennes?}

Les groupes Ferrières et Fontbouisse sont sans doute les mieux définis pour le Néolithique final méridional, en particulier grâce à leurs styles céramiques décorés. Ils conservent vraisemblablement leurs caractéristiques d'un bout à l'autre de leur aire d'extension et les faciès définis par X. Gutherz restent pour le moment indiscutés (Georjon et al., 1999, p. 286). Cependant, ils sont environnés de cultures où l'ornement est rare. Quelques ensembles représentent des faciès «exotiques» (Jallot, 1988, p. 82), soit parce que leur structure décorative ne correspond pas à un faciès déjà défini, comme la station du bois de Mintau à Calvisson, celle de Campguivard à Collias ou celle du Moulin-Villard (fosse 663) à Caissargues dans le Gard, soit parce que leurs éléments décoratifs sont déficitaires. Depuis quelques années maintenant, plusieurs études languedociennes envisagent que ces faciès «exotiques» forment, en rive ouest du Rhône, d'autres ensembles culturels illustrant des transitions douces du Ferrières au Fontbouisse, aux alentours de 3000 av. J.-C. (Jallot, 2003, p. 263).

Dans cette transition, les groupes culturels provençaux pourraient constituer des acteurs déterminants. À la suite des travaux de C. Georjon sur la céramique du site de la 


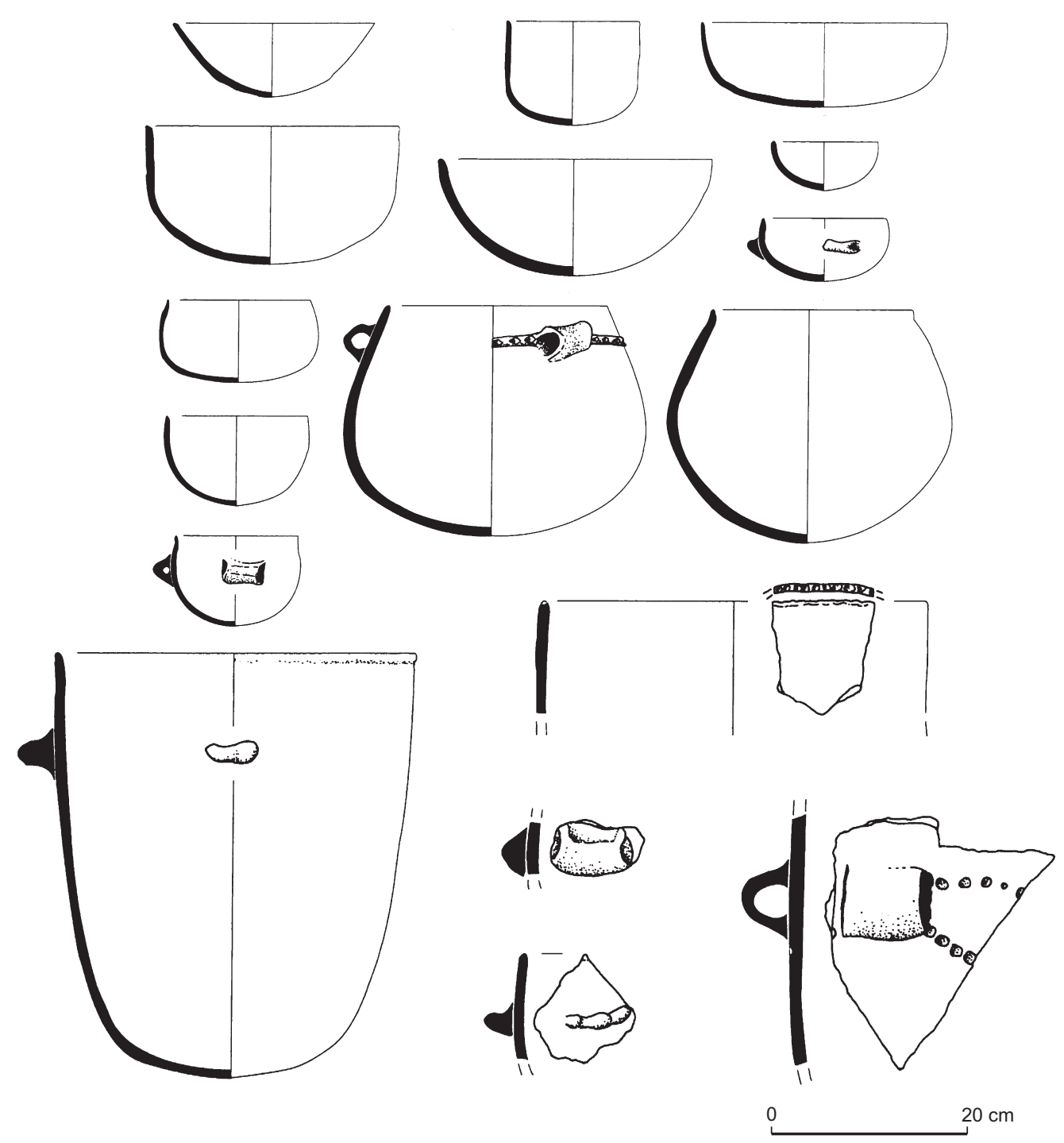

Fig. 10 - Synthèse des formes, des éléments de préhension et des décors céramiques du Néolithique final du style la Roquette (d'après Georjon et al., 1999, p. 284).

Roquette à Tresques dans le Gard, le répertoire morphologique et stylistique original a permis la définition d'un «faciès épi-Ferrières de style la Roquette» en basse vallée du Rhône (Georjon et al., 1999; Jallot, 2003, p. 262). Pour l'auteur, l'indigence des décors observés est notamment attribuable à la proximité de cultures céramiques peu ornées présentes en rive gauche du couloir rhodanien, telle que la culture du Couronnien (fig. 10). Si les influx diffusés par le Couronnien sont plus difficiles à percevoir que ceux d'une culture intégrant des décors céramiques bien typés comme le Fraischamp ou le Rhône-Ouvèze, plusieurs éléments couronniens seraient en effet suffisamment spéci- fiques pour être détectés à l'ouest du Rhône. Il s'agit, pour les formes céramiques, de vases très pansus à ouverture rétrécie et de quelques types distinctifs tels que les bassins ou tians, pour les décors, de cordons courts, et pour les éléments de préhension, d'organes tubulaires, en $\mathrm{H}$ ou en demi-bobine parfois prolongés par des cordons (Georjon et al., 1999, p. 285).

À l'occasion de la publication du site des Vautes à SaintGély-du-Fesc dans l'Hérault, L. Jallot caractérise un second «faciès épi-Ferrières des basses Cévennes et de la basse vallée de l'Hérault de style les Vautes» (Jallot, 2003). Comme à la Roquette, la production céramique se compose d'un 

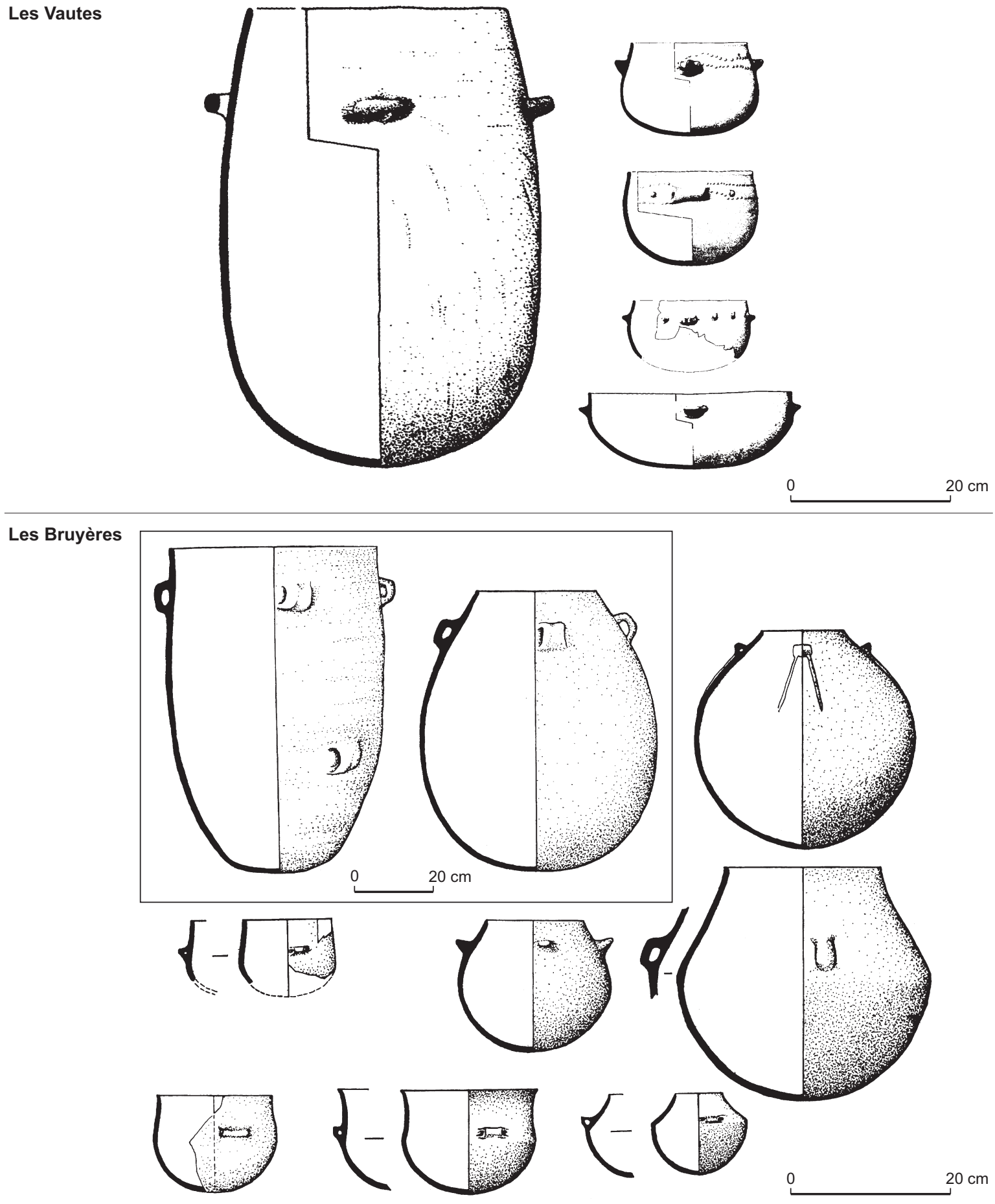

Fig. 11 - Synthèse des formes, des éléments de préhension et des décors céramiques du Néolithique final du style les Vautes (en haut, d'après Jallot, 2003, p. 256) et du style les Bruyères (en bas, d'après Gilles, 1975). 
répertoire morphologique simple et de décors se résumant à des cordons généralement lisses unissant les appliques, en tous points comparables aux productions couronniennes (fig. 11). Plus encore, les affinités typologiques et stylistiques avec des corpus provençaux sont telles que L. Jallot affirme que «la plupart de ces assemblages seraient qualifiés de Couronnien en rive est du Rhône» (ibid., p. 249). Ce type de composition stylistique est très bien attesté dans le Gard (Miraman, jardin ouvrier et voie urbaine sud à Nîmes, le bois de Mintau à Calvisson, Cabanelle à Saint-Maximin, Vallagrand à Vers-Pont-du-Gard, quartier de Crussol à PontSaint-Esprit).

En Ardèche, un «faciès épi-Ferrières de la basse vallée du Rhône de style les Bruyères ", semble également s'intercaler entre les traditions Ferrières et Fontbouisse (fig. 11). Il s'exprime à nouveau par des formes et des décors qui tendent à la simplification, comme plusieurs jarres pansues pouvant dériver elles aussi de certaines morphologies couronniennes (Beeching, 1980; Gutherz, 1984; Georjon et al., 1999, p. 285; Jallot, 2003, p. 258). Ce faciès est reconnu aux Bruyères (Saint-Julien-de-Peyrolas), à l'abri de la Vessignée (Saint-Marcel-d'Ardèche), au puits du Fakir (Balazuc), au Serre de Boidon ou au Ranc d'Aven (Grospierres). Son statut par rapport aux cultures languedociennes fait l'objet d'appréciations variées. Dans la littérature, il correspond, selon les auteurs, à un groupe autonome (Bordreuil, 1995 et 1998, p. 339), ou au faciès ardéchois du Fontbouisse (Gutherz, 1990, p. 240). Il a été initialement défini après la fouille du site éponyme à Saint-Julien-de-Peyrolas en 1975 par R. Gilles (1975), qui le considérait comme un ensemble de transition chronologique, technologique et culturel entre le Ferrières et le Fontbouisse. En sus des formes simples évoquant le Couronnien, cet ensemble des Bruyères adopte des formes à profil segmenté, des décors de pastilles appliquées et des impressions sur la lèvre et la panse, qui lui ont valu d'être à plusieurs reprises rapproché du RhôneOuvèze (Gutherz, Jallot, 1995; D’Anna, 1995a).

Ces trois faciès épi-Ferrières, dont les sites de la Roquette, des Bruyères et des Vautes constituent les principales références, posent ainsi, dans les marges orientales du Languedoc et en Ardèche, le problème de leurs relations avec les ensembles culturels provençaux tels que le Couronnien à la transition du $\mathrm{IV}^{\mathrm{e}} / \mathrm{III}^{\mathrm{e}}$ millénaire av. J.-C.

Précisons cependant que pour plusieurs archéologues, de nombreux arguments soutiennent la piste de la filiation directe du Ferrières au Fontbouisse sans intermédiaire extérieur (Jacobieski, 1988; Gutherz, Jallot, 1995, p. 242; Jallot, 2003, p. 246). Sur le site éponyme de Ferrières-Les-
Verreries, à la Baume de Ronze, à la grotte du Chemin de Fer à Boucoiran-et-Nozières ou encore dans la grotte des Meules à Rochegude (Gard), des exemples de transitions stratigraphiques ont été relevés entre le Ferrières et le Fontbouisse. Ils se traduisent souvent par le maintien de formes ancrées dans la tradition Ferrières, tels que les vases aux profils sinueux et l'apparition d'éléments préfigurant le Fontbouisse comme les vases carénés ou la mise en place d'un répertoire décoratif nettement plus riche. À cela s'ajoute le fait que certaines techniques décoratives sont relativement stables tout au long du cycle Ferrières-Fontbouisse : ce sont notamment les cannelures en guirlandes (arcs de cercle concentriques emboîtés), les incisions en chevrons, les décors de cordons et de pastilles. Enfin, la distribution des faciès de tradition Ferrières-Fontbouisse se recouvre assez bien, conformément à l'hypothèse de leur filiation. En revanche, il est encore aujourd'hui délicat d'envisager le séquençage de cette filiation et le phasage interne de chacun de ces groupes, depuis des étapes constitutives jusqu'à des phases de transition (Gutherz, Jallot, 1995, p. 243).

\section{Les composantes provençales du Néolithique final rhodano-alpin}

Il est difficile de ne pas évoquer la question des contacts entre les groupes culturels provençaux et ceux de la moyenne vallée du Rhône. Même si le manque de repères dans la région Rhône-Alpes fait encore obstacle aux comparaisons, A. Beeching a proposé en 2001, à l'occasion du colloque de Pordenone en Italie, le premier tableau chronologique et culturel du Néolithique final dans le bassin du Rhône moyen (Beeching, 2002). Dans les étapes successives de sa trame chronoculturelle, il situe dans un stade 2 (3400-2800 av. J.-C.) le groupe d'Allan (fig. 12), défini à la suite des fouilles qu'il a conduites sur le site éponyme du Jas des Chèvres à Allan dans la Drôme (Beeching, 1980 et 1986). Cet ensemble culturel, synchrone du Ferrières, est intégré au sein d'un vaste complexe désigné «Néolithique final rhodano-alpin» (fig. 13), dont la définition et la dénomination définitive, précise-t-il, attendent encore la publication de plusieurs séquences (Beeching, 2002, p. 78). Le groupe d'Allan est caractérisé par un héritage lointain du Chasséen dans le taux significatif du débitage laminaire, les prises biforées, la survivance de la carène et les formes à col. La morphologie distinctive est la jarre ou la marmite à ouverture très rétrécie et la tendance générale est aussi à l'allongement des récipients; on trouve en outre des vases tulipiformes, des préhensions en demi-bobine 


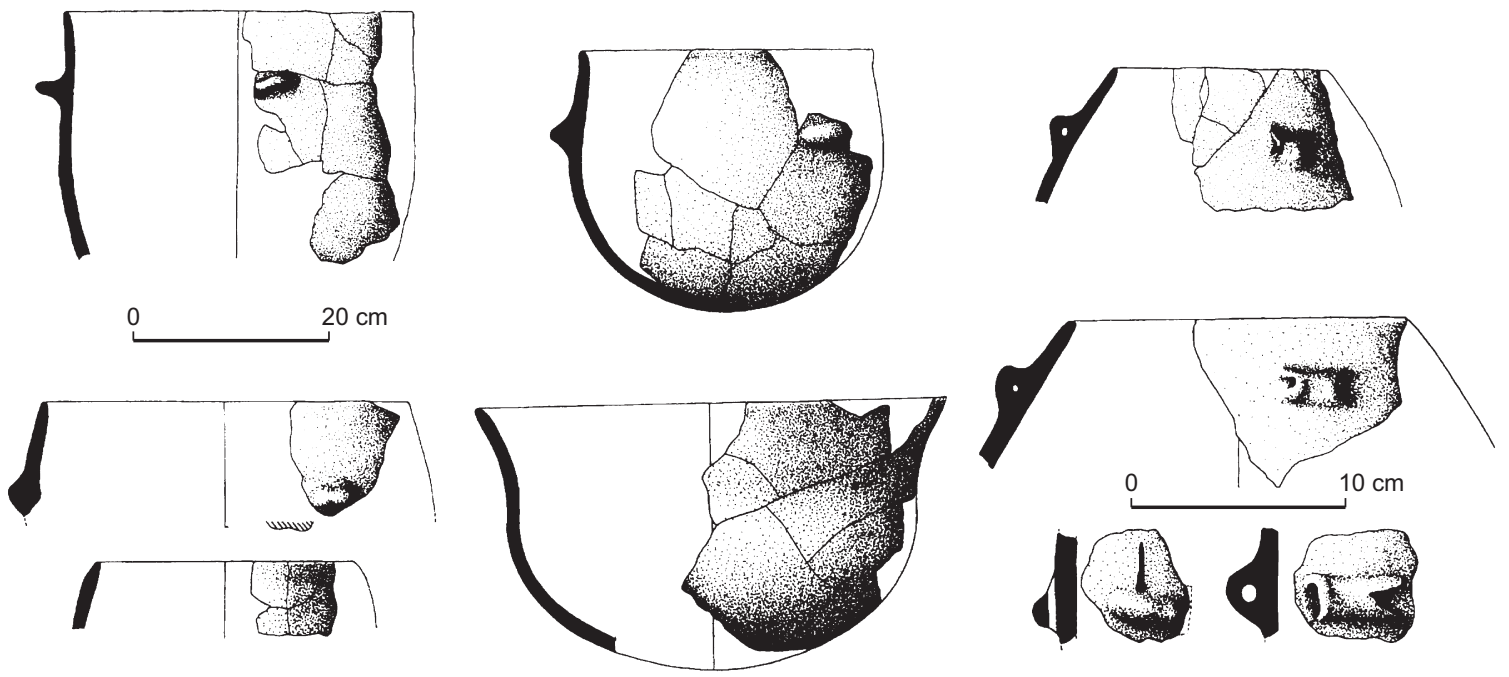

Fig. 12 - Synthèse des formes, des éléments de préhension et des décors céramiques du Néolithique final du groupe d'Allan (d'après Beeching, 2002, p. 79).
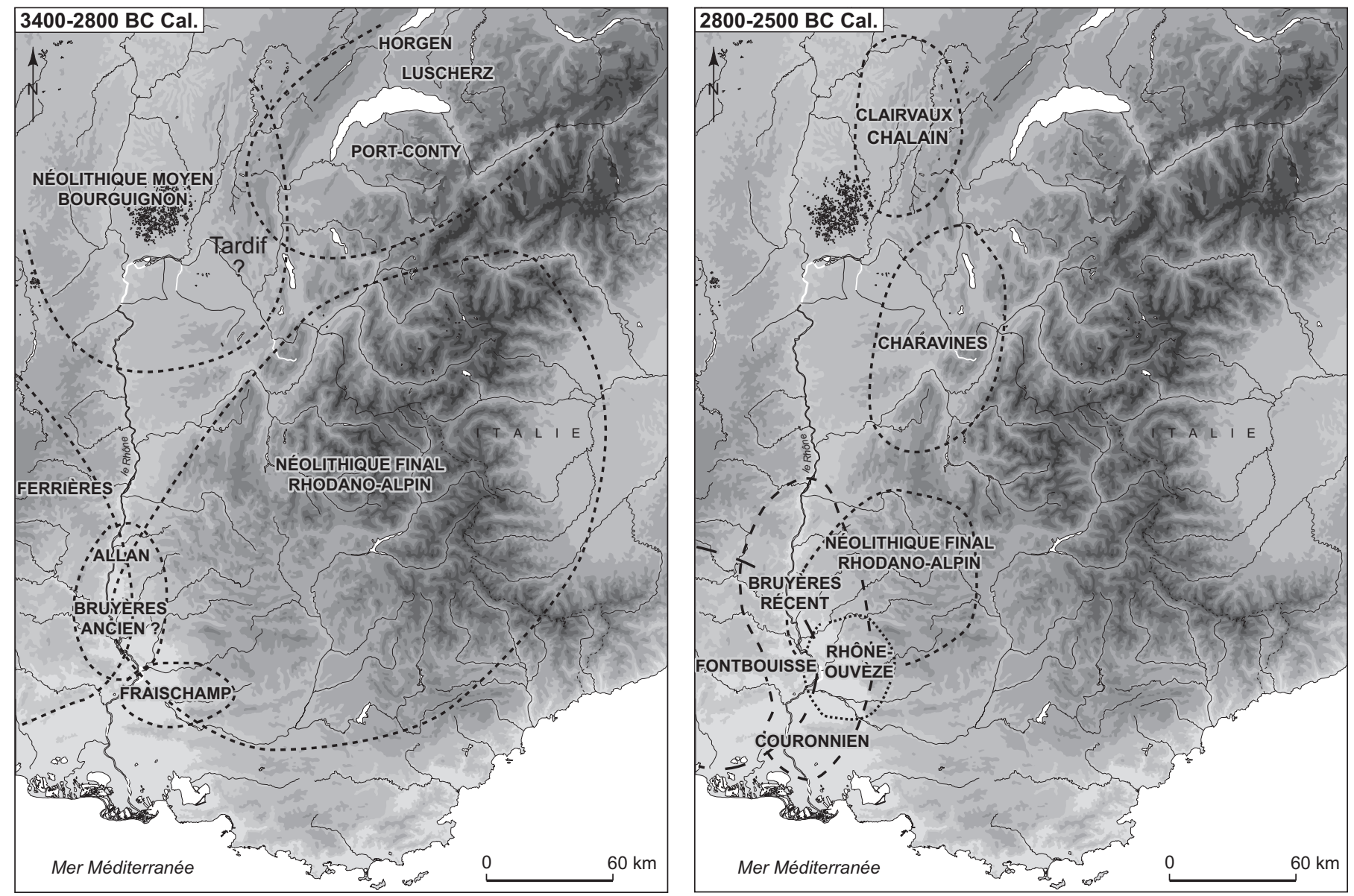

Fig. 13 - Lintégration des groupes culturels provençaux au complexe Néolithique final rhodano-alpin (d'après Beeching, 2002, p. 76). 
et des décors d'impressions sur la lèvre. Dans la Drôme, c'est avec le site de la grotte Chauve-Souris à Donzère que s'établissent les meilleurs parallèles, notamment avec les premiers niveaux archéologiques du gisement (Vital, 2006, p. 280). Le groupe d'Allan est plus ou moins contemporain de la phase ancienne des Bruyères en Ardèche et du groupe de Fraischamp en rive est. Selon A. Beeching et J. Vital, chacun de ces ensembles culturels pourraient livrer des productions relevant de schémas morphologiques communs (ibid.), car de nombreuses similitudes sont présentes en particulier dans les formes simples. De sorte que, dans le modèle proposé par A. Beeching, le Fraischamp, les Bruyères et le groupe d'Allan sont ralliés au sein du même complexe Néolithique final rhodano-alpin (Beeching, 2002, p. 76).

L'étape 3 de sa périodisation (2800-2500 av. J.-C.) est marquée, en vallée du Rhône proprement dite, par le prolongement du groupe des Bruyères en Ardèche, le développement du Fontbouisse en Languedoc, le prolongement du Couronnien et l'apparition du Nord-Vaucluse et du Rhône-Ouvèze en Provence (fig. 13). La Drôme voit, quant à elle, la disparition progressive du groupe d'Allan, remplacé par des influences encore non caractérisées précisément, mais qui restent schématiquement associées à une phase récente du Néolithique final rhodano-alpin. Là encore, dans sa proposition de périodisation, A. Beeching établit un parallèle entre le Néolithique final rhodano-alpin et le Néolithique final de Provence, car la forme générale des récipients typiques du groupe Nord-Vaucluse (vases profonds droits cylindriques ou tulipiformes), les éléments de préhension (fréquents boutons et mamelons prismatiques, parfois disposés par quatre près de l'ouverture), tout comme la faiblesse, sinon l'absence des décors en creux de la céramique de l'hypogée des Crottes à Roaix, peuvent évoquer l'environnement culturel de la moyenne vallée du Rhône (Beeching, 2002, p. 81). Il précise d'ailleurs que «l'ensemble de la Drôme et de la Provence est marqué par des influences que l'on peut schématiquement associer au courant Néolithique final rhodano-alpin» (ibid.).

La fin de cette étape 3 (aux alentours de 2600-2500 av. J.-C.) est caractérisée par la présence ponctuelle du groupe de Fontbouisse dans le bassin du Rhône moyen: la séquence supérieure Néolithique final de la grotte de la Chauve-Souris, à Donzère dans la Drôme, livre un corpus caractéristique dans les thématiques décoratives et dans le cortège des formes. Des parallèles entre les phénomènes culturels de ce secteur géographique et les ensembles provençaux sont par ailleurs envisagés, puisque la stylistique des ornementations de la Chauve-Souris est semblable à celle des gisements de Provence occidentale attribués au Rhône-Ouvèze: en particulier les séries d'incisions horizontales surmontant des lignes brisées (Vital, 2006, p. 283).

Ces premières observations révèlent avec force, qu'au nord et à l'ouest de la Provence, les groupes Couronnien, Fraischamp, Rhône-Ouvèze et Nord-Vaucluse ont pu participer aux mécanismes de changement culturel au premier rang desquels le passage du Ferrières au Fontbouisse et la constitution de nouvelles entités dans le secteur drômois. Il est pourtant encore bien difficile de démêler ces apports respectifs car, dans la Drôme et en Ardèche, les processus de périphérisation aux marges géographiques de cultures très expansives tels le Ferrières ou le Fontbouisse entravent la compréhension des interactions entre les nombreuses entités culturelles. Dans cette zone en effet, de multiples influences se succèdent en provenance d'axes géographiques divers: depuis le Languedoc, mais certainement aussi depuis le Jura et la Suisse, de sorte que la part de chacun des groupes provençaux mobilisés, leur effet respectif et l'éventuelle diversité des groupes culturels générés par ces imbrications constituent autant de pistes à approfondir.

\section{LES APPORTS MÉRIDIONAUX DANS LE NÉOLITHIQUE FINAL DE L'AXE SAÔNE-RHÔNE}

Encore plus au nord et à l'est, la structuration culturelle autour de l'axe Saône-Rhône au III ${ }^{\mathrm{e}}$ millénaire av. J.-C. est marquée par des effets de frontières, par des échanges et par des limites d'extension maximales de cultures particulièrement actives, tels le groupe des Treilles et le Ferrières dans un premier temps, puis le Cordé et le Fontbouisse ensuite. Une présentation préliminaire rappelle les grandes lignes de la trame chronoculturelle de cette zone géographique et montre que, dans la configuration actuelle, les phénomènes culturels de Provence sont absents dans les schémas de mise en place et de construction.

\section{Le rôle du Languedoc et la structuration des phénomènes culturels au nord des Alpes}

Dans un cadre géographique qui s'étend de la Suisse orientale au nord, au Léman et au Valais au sud, et de la Combe d'Ain à l'ouest, jusqu'à l'Italie du Nord à l'est en passant par la région des Trois-Lacs, les groupes du Néolithique final méridional semblent avoir joué un rôle prépondérant. Plusieurs bilans présentent dans le détail le canevas chronoculturel élaboré pour ces régions. Le lecteur peut s'y référer 


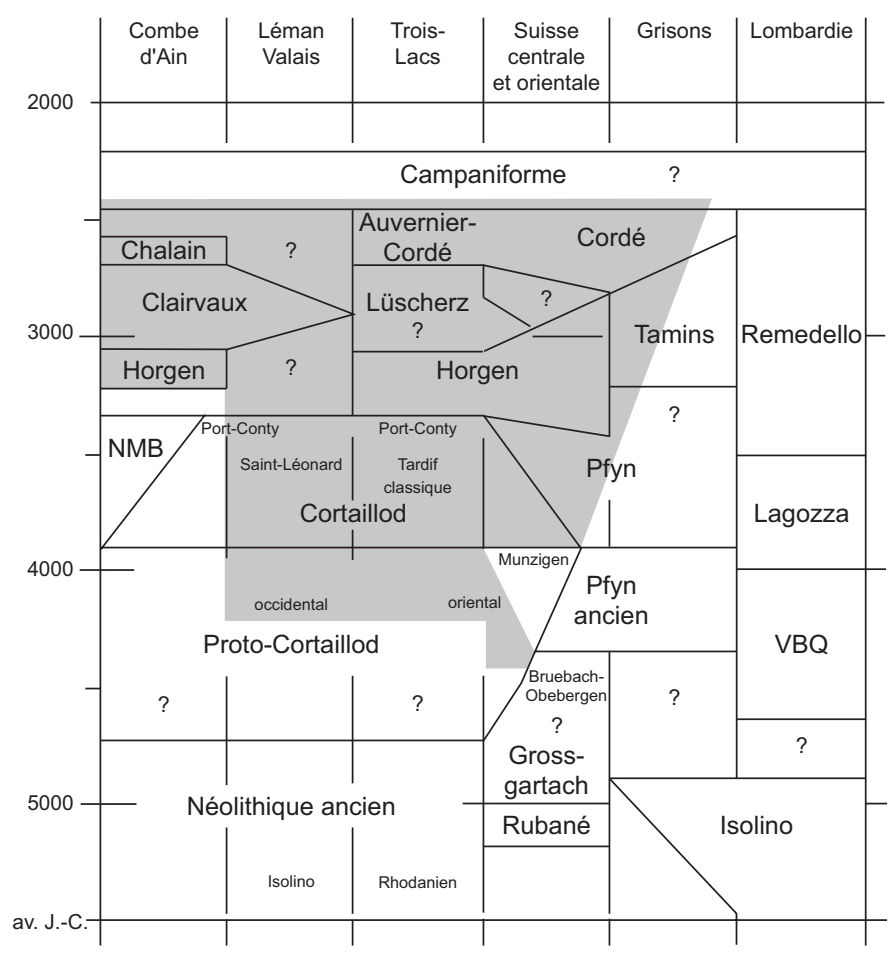

Fig. 14 - Tableau chronologique du Néolithique suisse et des régions limitrophes de France et d'Italie (d'après Honegger, 2001, p. 18).

pour tous compléments (Thévenot et al., 1976; Pétrequin et al., 1987-1988; Ramseyer, 1987; Wolf, 1990-1991 et 1993; Giligny, 1993; Giligny et al., 1995; Honegger, 2001, p. 20-23 ; Bailly, 2002).

Le Néolithique final débute avec le groupe Horgen, qui occupe tout le plateau Suisse. Cette culture serait issue d'une évolution sur place du Pfyn, la transition se situant vers 3500-3350 av. J.-C. (fig. 14). Depuis sa zone d'origine, le Horgen va s'étendre rapidement vers la région des Trois-Lacs, pour atteindre ensuite la Combe d'Ain vers 3200 av. J.-C. et le versant nord des Alpes. Pour l'instant, il n'existe pas de sites dans le bassin lémanique et dans le Valais qui permettent d'avancer que le Horgen a également touché ces régions. En Suisse orientale, le Horgen est attesté jusqu'à 2800 av. J.-C., alors qu'en Suisse occidentale la période comprise entre 3100 et 2950 reste encore mal cernée. Le répertoire des formes classiques du Horgen se traduit par des vases de contour simple aux parois droites à fond plat saillant, des décors digités, de doubles ou triples incisions horizontales parallèles, de cannelures ou de cordons horizontaux sous le bord. Dès 3200 av. J.-C., l'originalité stylistique de certaines productions à pâte fine et aux surfaces lustrées dotées de décorations de pastillage au repoussé ou d'incisions en guirlandes est mise sur le compte des premiers contacts avec le Néolithique final Ferrières du midi de la France (Giligny et al., 1995, p. 315). À la transition du IV ${ }^{\mathrm{e}}$ au III ${ }^{\mathrm{e}}$ millénaire, un renouvellement culturel total s'opère sous le poids de ces interactions. Cela se traduit notamment dans la céramique: la plastique décorative, avec les boutons, les tétons, les languettes et les cordons uniques sont typiques du Ferrières, alors que les traditions céramiques locales ne sont peu ou prou pas conservées (ibid., p. 319).

$\mathrm{Au}$ début du III $^{\mathrm{e}}$ millénaire, se développent plusieurs groupes régionaux anciennement réunis sous le terme de «civilisation Saône-Rhône»: il s'agit des groupes du Lüscherz, de Clairvaux, de l'Auvernier-Cordé et du Néolithique final lémano-valaisan (Pétrequin et al., 19871988, p. 58-70). Dans un intervalle s'étendant de 2900 à 2700 av. J.-C., le secteur des Trois-Lacs est occupé par la culture de Lüscherz, dont l'expansion s'étend plus à l'est jusqu'au lac de Zoug (Honegger, 2001). La production céramique de ce groupe se caractérise par des récipients de formes simples droits ou globulaires à fond rond. Pour cette période, le bassin lémanique reste peu documenté, mais quelques indices montrent que l'évolution culturelle est proche de celle du groupe de Clairvaux qui apparaît en Combe d'Ain. Les influences de la façade méditerranéenne de la France se poursuivent de manière intensive dans ces deux groupes (fig. 15). Les décors de cannelures en guirlandes reliant des préhensions, tout comme le développement des jarres à deux cordons horizontaux ou à cordons multiples couvrant toute la panse et les décors de chevrons incisés évoquent à nouveau les ensembles culturels des Treilles et de Ferrières (Pétrequin et al., 1987-1988, p. 30; Giligny et al., 1995, p. 316).

En Suisse orientale, le Cordé apparaît vers 2800 av. J.-C., en renouvelant la totalité du répertoire de la culture matérielle par rapport au Horgen qui le précède. Il touche, par son ampleur, une bonne partie du territoire de l'Europe centrale et occidentale. Cette influence est directe, due à des déplacements de populations, ou indirecte, propagée par la dissémination d'éléments caractéristiques de la culture matérielle. Le mobilier récurrent du Cordé est représenté par des gobelets à fond plat décorés à la cordelette. Avec son expansion, les apports méridionaux semblent s'estomper et sont progressivement remplacés au profit de ceux venant de l'est de l'Europe. Rapidement, le Cordé atteint la région des Trois-Lacs, où plusieurs sites stratifiés permettent de suivre son influence croissante sur la céra- 


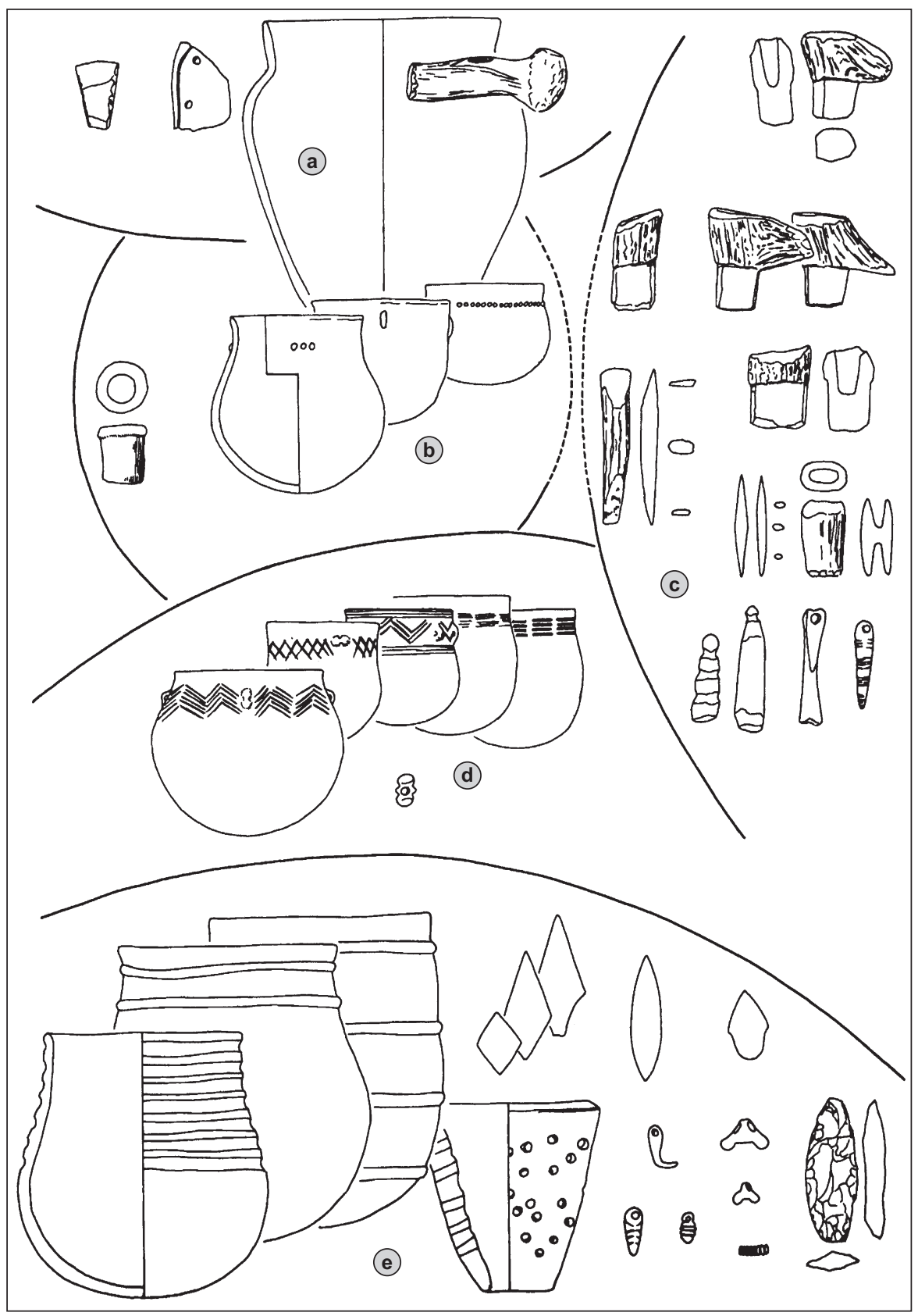

Fig. 15 - Le groupe de Clairvaux et les composantes culturelles qui ont influé sur sa formation: $\boldsymbol{a}$, composantes nord-orientales Seine-Oise-Marne récent; $\boldsymbol{b}$, traditions régionales Cortaillod Port-Conty; , traditions orientales Cortaillod et Horgen; $\boldsymbol{d}$, composantes méridionales secondaires (les Treilles-Ferrières); $\boldsymbol{e}$, composantes méridionales primaires (les Treilles-Ferrières) (d'après Pétrequin et al., 1987-1988, p. 59).

mique de tradition locale (Honegger, 2001). Dès 2700-2650 av. J.-C., cela est lisible dans le groupe d'Auvernier nommé Auvernier-Cordé pour souligner le métissage entre le style local et celui de la céramique Cordé. Les transformations sont en effet radicales. Les modèles de décor, de types morphologiques, de techniques de montage et de finition du Cordé sont adoptés par les groupes allogènes. Le modelage remplace la technique de l'applique. De même, les formes segmentées à profil en $\mathrm{S}$ et fond plat prennent la place des formes globulaires ou droites à fond rond (Giligny et al., 1995, p. 316). Plus au sud, le Cordé atteint la Combe d'Ain, où il donne naissance au groupe de Chalain aux alentours de 2600-2500 av. J.-C. En revanche, dans le bassin lémanique et en Valais, il ne semble pas exercer son influence.

Depuis quelques années, les chercheurs ont attiré l'attention sur l'existence, dans le reste des Alpes, d'une 


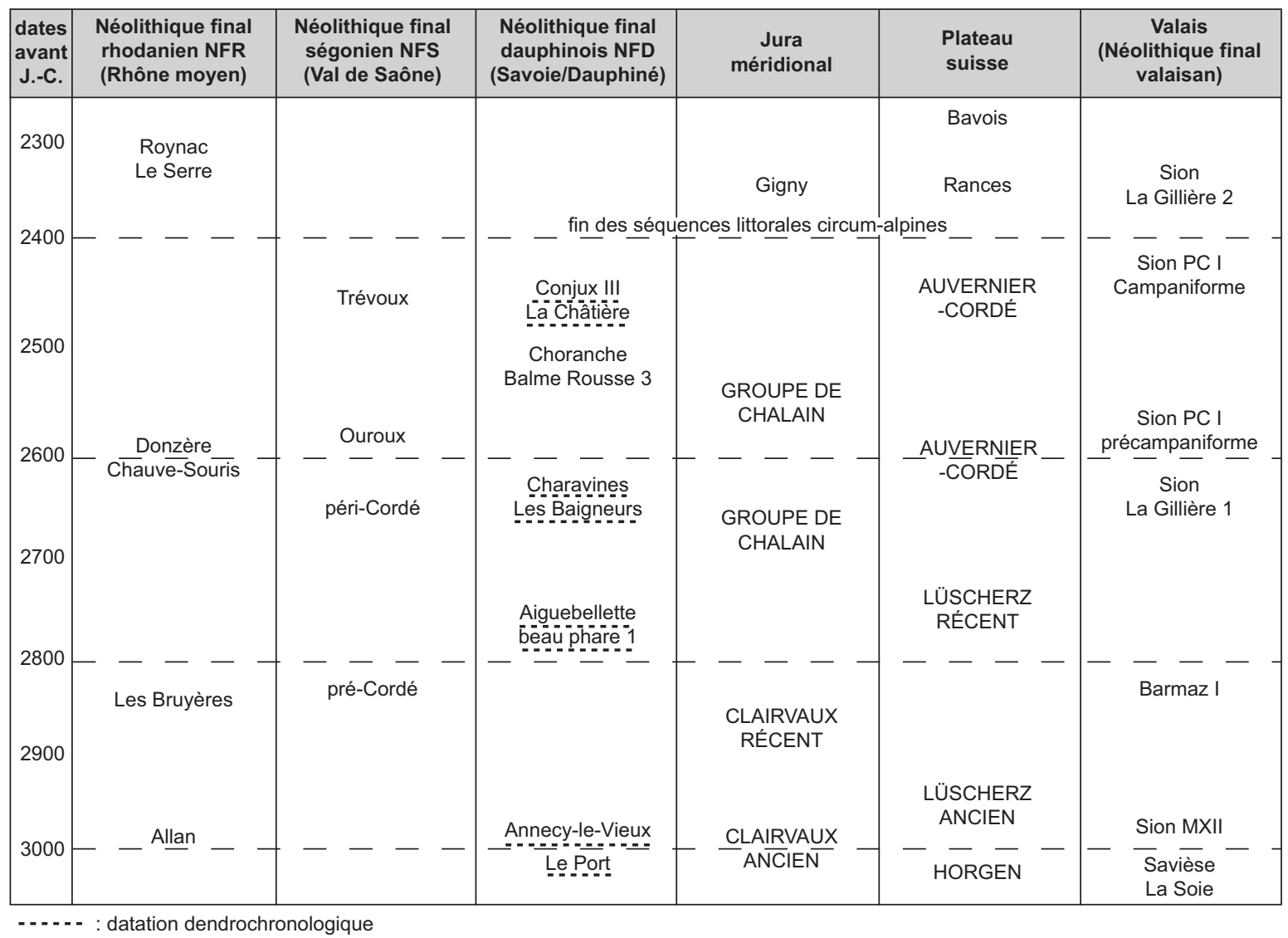

Fig. 16 - Proposition de tableau chronoculturel régional pour le Néolithique final de l'axe Saône-Rhône (d'après Bailly, 2002, fig. 1.33).

dernière composante culturelle porteuse de nouveaux apports méridionaux au moment où le Cordé centralise pourtant toutes les influences (Pétrequin et al., 1987-1988, p. 72). Le site de la station des Baigneurs à Charavines (Isère) en constitue la référence principale. Dans sa thèse de Doctorat, M. Bailly assimile cette composante à un complexe d'attente, le «Néolithique final dauphinois» (fig. 16), regroupant au sud du Rhône, les sites de Savoie et du Dauphiné, correspondant à de nombreuses occupations littorales datées par la dendrochronologie (Bailly, 2002, p. 50-51). Le Néolithique final dauphinois est initialement subdivisé en trois étapes (Wolf, 1993, p. 235). Dans une première phase de 3100 à 2700 av. J.-C, les sites d'Annecyle-Vieux, d'Aiguebelette II, en Haute-Savoie et la grotte des Sarrasins à Pommerol dans la Drôme sont mis en parallèle avec le style de Clairvaux identifié dans le Jura méridional. Un horizon moyen (2750-2650 av. J.-C.) est rapproché des groupes Auvernier-Cordé et Chalain reconnus en Suisse et dans le Jura. Enfin, les sites de Conjux III, la Chatière, Meimart I et Charavines en Isère forment le groupe le plus récent, aux alentours de 2650-2400 av. J.-C.

Le Campaniforme parachève le Néolithique en se manifestant à partir de 2500 av. J.-C. dans l'ensemble de ces régions où il disparaît aux alentours de 2300 av. J.-C., relayé par les premières cultures de l'âge du Bronze.

Pour mieux cerner l'évolution culturelle du plateau Suisse et des régions avoisinantes, la parure et les flèches sont aussi très efficaces (fig. 15). Elles sont en effet d'excellents marqueurs des rapports avec le midi de la France. Les pendeloques striées à pointes, les pendeloques à ailettes, les perles segmentées en os et les perles en stéatite, en calcaire ou en marbre sont des formes récurrentes dans les ensembles jurassiens de Clairvaux et de Chalain, dont l'appartenance culturelle et géographique est clairement languedocienne (Pétrequin et al., 1987-1988, p. 12; BargeMahieu, Bordreuil, 1990-1991; Wolf, 1990-1991). Certaines 
de ces parures sont des importations, mais il existe aussi des imitations ou des adaptations locales (Giligny et al., 1995, p. 318).

Dans la Combe d'Ain ou dans la région des TroisLacs, la coexistence de flèches triangulaires simples et de flèches losangiques vers 3200-3100 av. J.-C. souligne la pénétration précoce des premiers influx méridionaux en pleine ambiance horgenienne. Aux alentours de 3000-2900 av. J.-C., lorsque dominent les éléments céramiques du Clairvaux, directement apparentés aux faciès du Ferrières, l'essentiel des flèches est de type losangique, comme dans la zone d'origine des styles céramiques. Enfin, entre 2700 et 2500 av. J.-C., on retrouve des modèles de flèches losangiques méridionales, avec des variantes plus nombreuses, comme les armatures lancéolées, issues de contacts avec la moyenne vallée du Rhône (Pétrequin et al., 1987-1988, p. 38-43; Giligny et al., 1995, p. 318).

\section{Que faire de la Provence?}

Dans le schéma tel qu'il est admis aujourd'hui, l'espace géographique correspondant à l'arc jurassien et alpin, se trouve ainsi soumis tout au long du cycle néolithique final à des influx méridionaux importants. Entre la Saône et le Rhône, l'aire spatiale est ouverte sur l'axe rhodanien, couloir naturel Sud/Nord pour les échanges et les déplacements. Les relations entre le Midi méditerranéen, le plateau Suisse et ses régions avoisinantes sont attestées par la diffusion des cultures du Midi-Pyrénées (groupe des Treilles de la région des Grands Causses et du Rouergue) et du Languedoc oriental (Ferrières et Fontbouisse). Ces dernières sont notamment perçues comme des composantes majeures dans la genèse et l'évolution des différents groupes culturels. Par exemple, vers 3200 av. J.-C., en Suisse occidentale et dans le Combe d'Ain, la culture de Horgen reçoit en partie des infiltrations culturelles originaires des régions Ferrières (Giligny et al., 1995, p. 319). Deux siècles plus tard, en Suisse occidentale, le développement du groupe de Lüscherz est interprété comme la conséquence «d'une régionalisation du courant méridional Ferrières » (Giligny et al., 1995, p. 315), tandis qu'en Combe d'Ain vers 2900 av. J.-C., le groupe de Clairvaux marquerait l'expansion septentrionale extrême de poussées culturelles en liaison avec le déplacement de petits groupes humains issus du Ferrières ou du groupe des Treilles (Pétrequin et al., 1987-1988, p. 79; Giligny, Michel, 1995; Pétrequin, 1997; Honegger, 2001, p. 22 et p. 190). En bref, l'ensemble du secteur représente, au moins avant l'arrivée du Cordé, «l'aboutissement d'une périphérisation du complexe culturel Ferrières-les Treilles ", avec des rapports par ailleurs marqués en direction du Fontbouisse vers 26002500 av. J.-C. (Giligny et al., 1995, p. 316).

Bien que les chercheurs aient pris très tôt toute la mesure de ces interférences entre les groupes du Néolithique final méridional et ceux du nord des Alpes (Thévenot et al., 1976; Pétrequin et al., 1987-1988; Wolf, 1993; Ramseyer, 1987; Giligny, 1993), les cultures liées au secteur provençal n'apparaissent pas, même de façon secondaire, dans les schémas de construction (fig. 17). Tout au plus, peut-on lire que les modèles de flèches losangiques découvertes dans le groupe de Clairvaux sont potentiellement issus de contacts avec le Vaucluse (Giligny et al., 1995, p. 318).

Plusieurs pistes actuelles tendent à mettre en évidence que le Couronnien, le Fraischamp, le Nord-Vaucluse et le Rhône-Ouvèze ont pu s'étendre hors de leurs limites régionales, notamment en Languedoc et en Ardèche dans l'hypothèse d'une transition progressive du Ferrières au Fontbouisse et dans la Drôme, où ils pourraient influer sur le courant néolithique final rhodano-alpin (voir supra, p. 276). À cela s'ajoute le fait que la Provence constitue, tout autant que le Languedoc, une zone propice aux échanges et aux déplacements du fait de la proximité du couloir rhodanien. D'ailleurs, la diffusion des poignards de Forcalquier (Alpes-de-Haute-Provence) est aujourd'hui confirmée en Suisse dans les groupes de Horgen, de Lüscherz et de l'Auvernier-Cordé (Honegger, 2006, p. 52).

Dès lors, entre des groupes culturels languedociens très expansifs et d'autres septentrionaux particulièrement réceptifs, quel statut accorder à la Provence? Constituet-elle un pôle géographique isolé de ces mouvements de diffusion ou un lieu de passage, une sorte d'intermédiaire d'influences du sud vers le nord, d'autant que les groupes culturels provençaux sont particulièrement tributaires des apports du Languedoc ? Aussi, pouvons-nous évaluer autrement l'implication des groupes provençaux en envisageant qu'ils ont, comme ceux de la rive ouest du Rhône, participé à la construction des cultures de la sphère circumalpine?

L'apparition dans les sites d'Isère et de Savoie attribués au Néolithique final dauphinois, à la fois dans les styles morphologiques et dans le répertoire décoratif, d'influx septentrionaux du Cordé montre l'importance des nouveaux réseaux d'échange ouverts selon un axe de diffusion nord/sud. Alors qu'il est encore difficile d'estimer les capacités expansionnistes des groupes culturels provençaux, cette dernière observation révèle avec force la possibilité qu'ils aient pu être également perméables à certains de ces influx. 


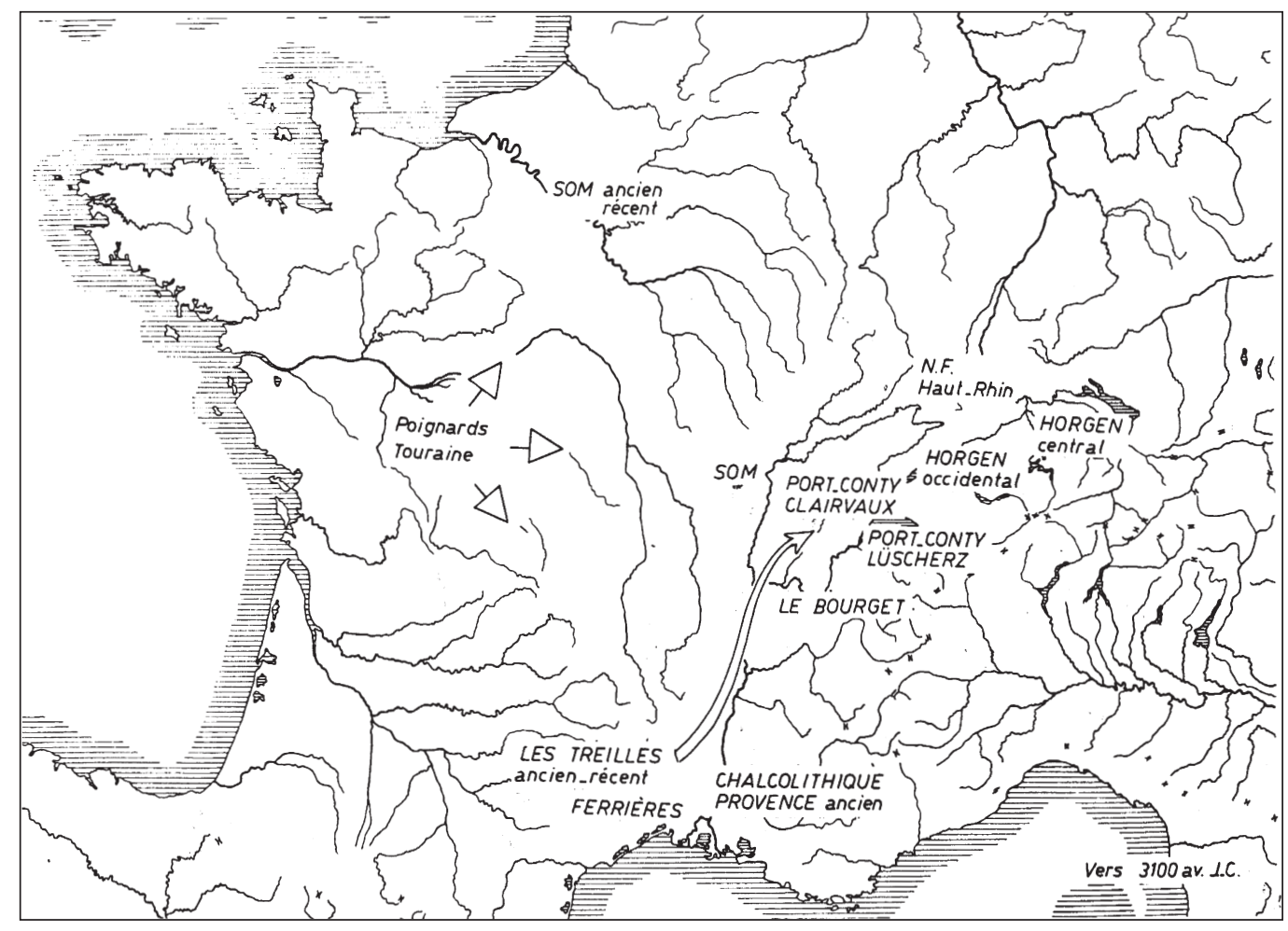

Fig. 17 - La mise en place des groupes du Néolithique final qui ont influencé le Jura méridional, aux environs de 3100 av. J.-C. (d'après Pétrequin et al., 1987-1988, p. 59).

\section{LES PHÉNOMÈNES DE TRANSGRESSION GULTURELLE EN PROVENCE}

C'est en effet, une fois de plus, en Provence, qu'il est possible de constater que les groupes culturels sont particulièrement sujets aux influences extérieures, provenant comme on l'a déjà vu, soit de l'ouest depuis le Languedoc, mais aussi de l'est depuis l'Italie du Nord. Le phénomène campaniforme, dont les aires d'émergence restent à débattre, s'intègre également en Provence dans les groupes locaux, avant de devenir autonome. Il est donc important de s'interroger sur la place et la nature des apports italique et campaniforme en rive gauche du Rhône, qui peuvent éventuellement constituer des éléments de compréhension supplémentaires à l'évolution et la transformation des groupes autochtones.

\section{L'Italie septentrionale et centrale et le Néolithique final des Préalpes du Sud}

Très tôt dans l'histoire de la recherche, des liens avec l'Italie du Nord ont été perçus. Ces contacts sont matéria- lisés par des marqueurs tels que les céramiques metopale (fig. 18 et 19) découvertes à l'abri de Pendimoun à Castellar dans les Alpes-Maritimes (Binder, 2003), le poignard en cuivre emblématique du Remedello trouvé au mas des Gavots à Orgon à l'embouchure de la Durance, dans les Bouches-du-Rhône (Courtin, Sauzade, 1975), les gravures d'armes comparables représentées dans l'abri sous roche des Oullas dans la haute vallée de l'Ubaye (Alpes-deHaute-Provence) (Rossi, Gatigglia, 1998) et la circulation de matières siliceuses. Dans la zone des gravures du mont Bego (haute vallée de la Roya, Alpes-Maritimes), des figurations de poignards à soie courte pourraient éventuellement correspondre à des pièces remedelliennes n'ayant pas conservé leur manche en matière organique (Saulieu, 2004; Vital, 2006). Le phénomène des pointes lithiques dites de Sigottier, retrouvées dans les grottes de Sigottier dans la vallée du Buëch et sur les sites du col des Tourettes à Montmorin dans les Hautes-Alpes (Muret, 1991 et 1996), présente également quelques similitudes avec les armatures Remedello de la plaine padane centro-orientale (Durand, 1999; Morin et al., 2005 et 2006). Enfin, les travaux de E. Sangmeister ont montré que les variétés de cuivre carac- 
Remedello

(0)
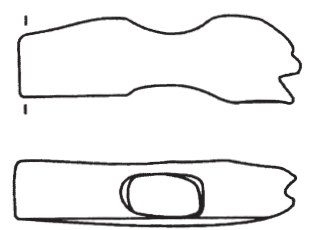

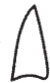

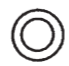

$[0]$
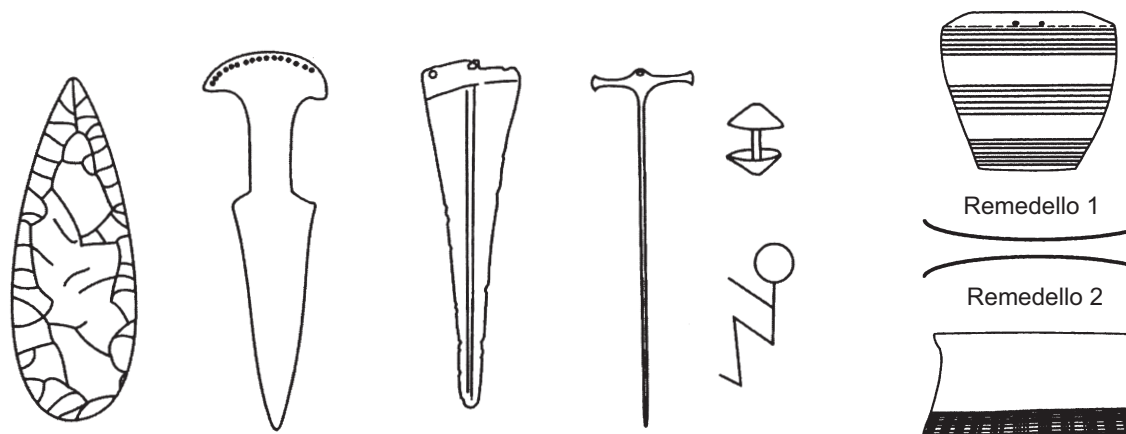

Remedello 1
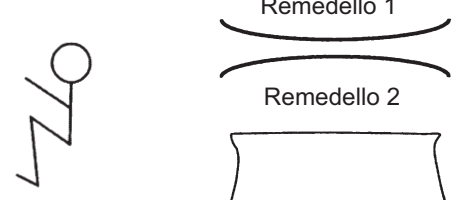

Remedello 2
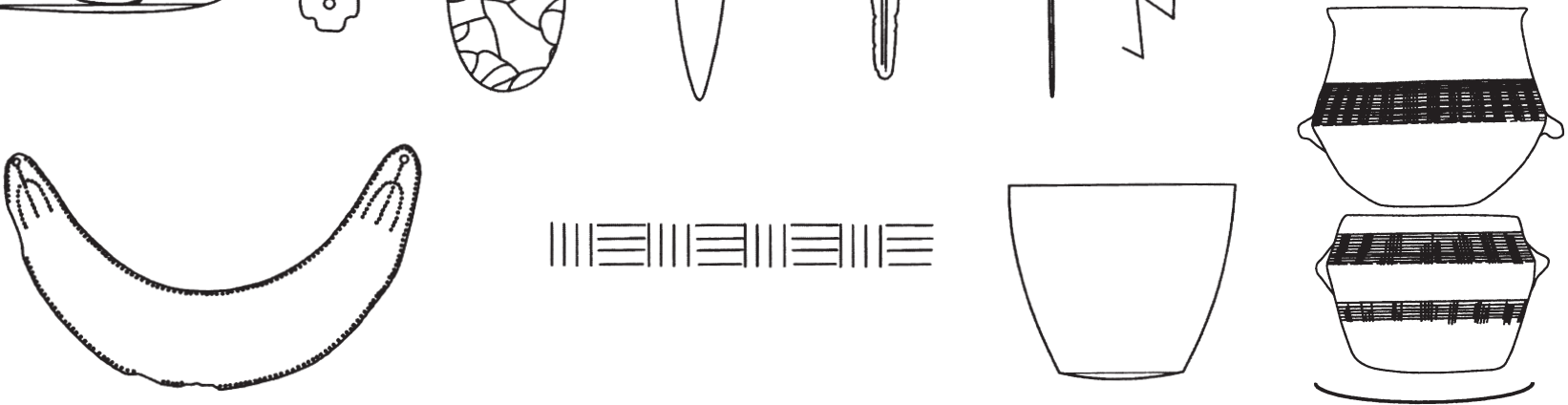

Rinaldone

(1) $\theta_{-}^{-}$
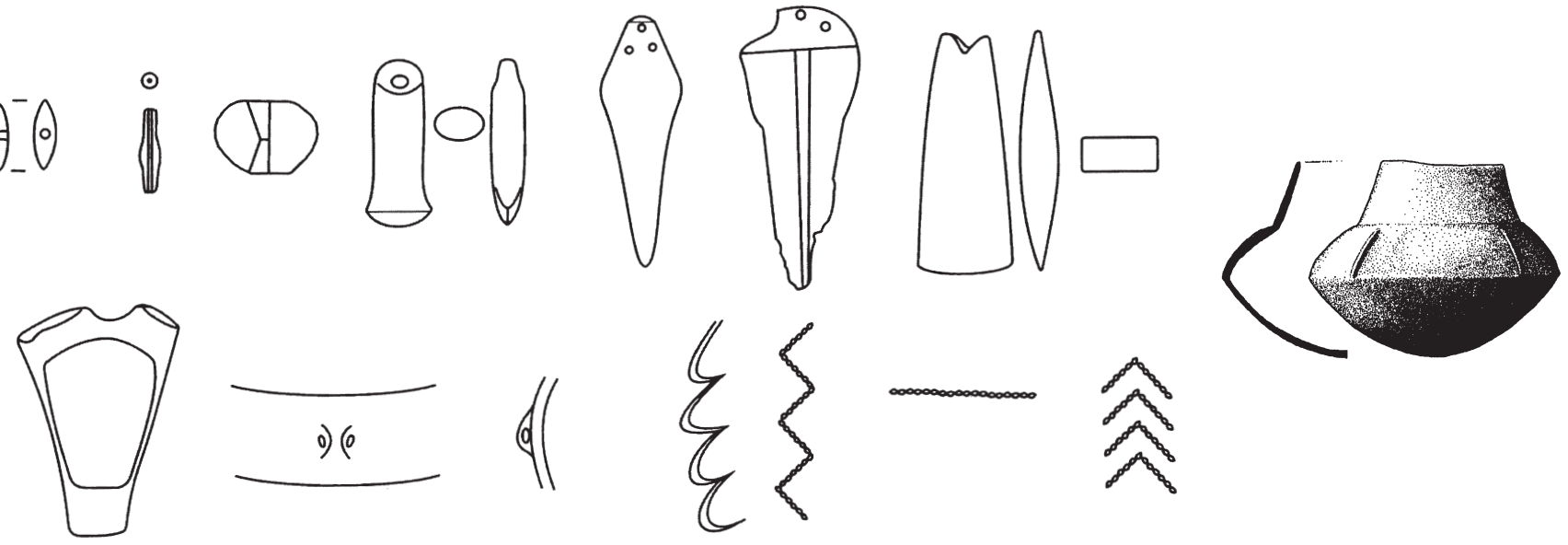

Fig. 18 - Caractéristiques métalliques, lithiques, de parure, céramiques et sépulcrales des groupes italiens Remedello et Rinaldone (d'après Steiniger, 2005, p. 294 et Vital, à paraître).

téristiques de Remedello étaient bien attestées dans le midi de la France (Sangmeister, 2005).

La culture de Remedello est localisée en Italie septentrionale au nord de la Lombardie, entre le lac de Garde, le Haut-Adige et la plaine Padane (Cornaggia Castiglioni, 1971). Elle correspond à des contextes funéraires (Steiniger, 2005). Récemment, R.-C. De Marinis et A.-L. Pedrotti ont proposé une chronologie en deux phases à partir du site éponyme de Remedello-Sotto: un premier épisode ancien de 3400 à 2800 av. J.-C. et un second récent de 2900/2800 à 2400 av. J.-C. (Marinis, Pedrotti, 1997). L'Italie a joué un rôle précurseur dans l'évolution de l'exploitation du cuivre. Les cultures du Chalcolithique de ce pays, plus particu- lièrement celle de Remedello, ont été les premières à être capables de réaliser à grande échelle la transformation des cuivres gris. Les contacts avec la Provence sont donc mis directement en parallèle avec la forte influence des groupes transalpins sur la métallurgie languedocienne, dont on sait qu'elle a conduit à la chalcolithisation du reste de l'hexagone (Strahm, 2005, p. 31; Rossi, Gattiglia, 2005). C'est durant la deuxième étape du Remedello qu'une expansion en direction de l'arc alpin et vers le sud-est de la France a pu se produire.

En Provence, le contexte culturel dans lequel s'insèrent ces influences est encore peu connu. Les gravures ou encore les pointes de Sigottier ne sont pas attribuées: elles 


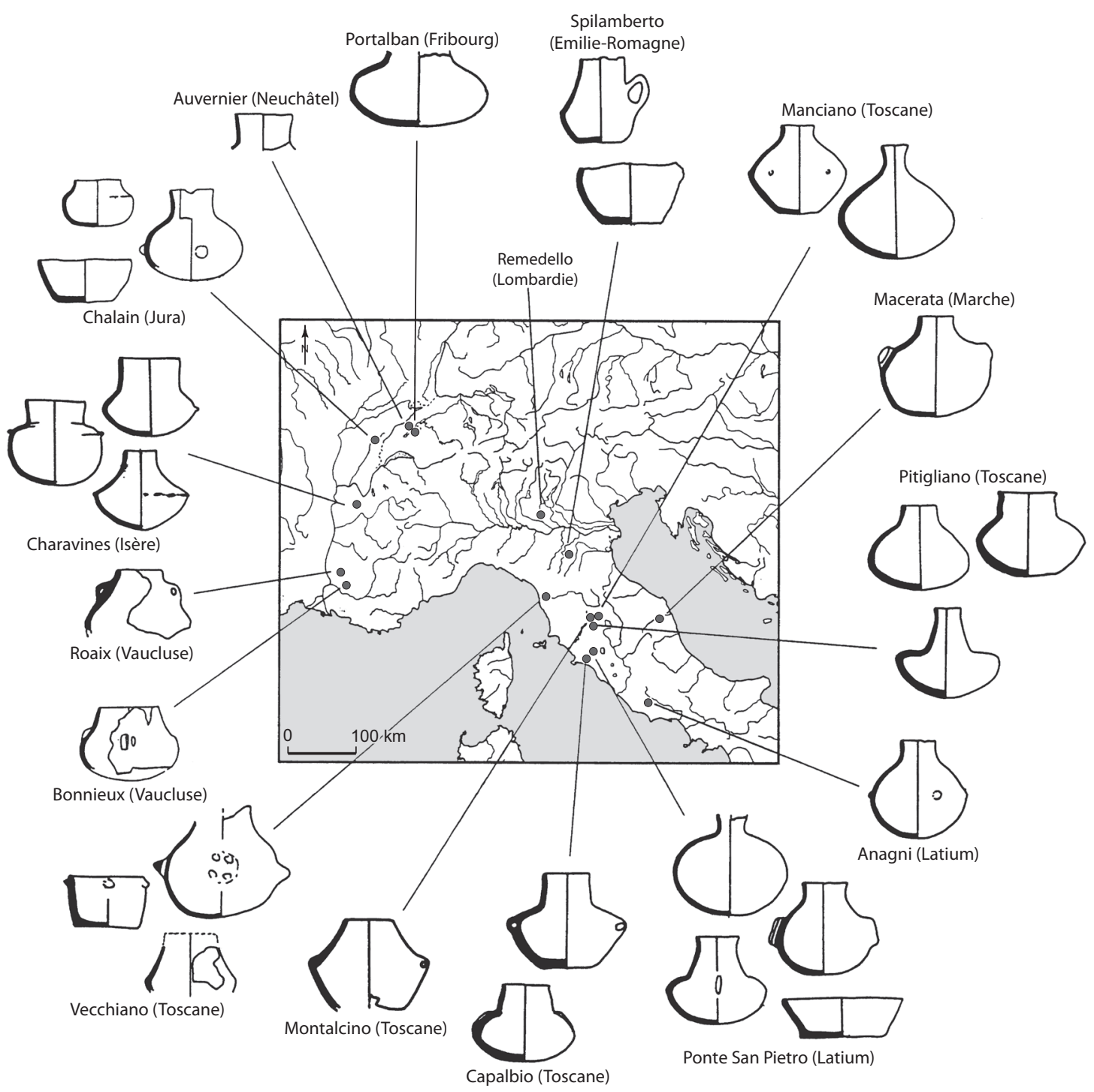

Fig. 19 - Diffusion des céramiques d'Italie centrale au III millénaire av. J.-C. (d'après Pétrequin in Bailly, 2002, fig. 1.24).

sont associées à de nombreuses séries lithiques qu'il est délicat de classer précisément. La quasi-absence de céramique est également un obstacle à une bonne compréhension de la situation (D’Anna, 1999, p. 156). Depuis quelques années, des recherches ont été engagées dans le secteur des Hautes-Alpes, qui focalise la plupart des découvertes liées à l'introduction italienne (Morin, 2000 et 2005; Morin et al., 2000). Une première hypothèse semble émerger selon laquelle il existerait probablement dans le secteur périalpin, un Néolithique final local à la fois perméable aux intrusions du Remedello et correspondant à une extension des cultures périphériques d'obédience rhodanienne.
Ainsi, les pointes de flèche de Sigottier sont relativement proches de certains exemplaires provenant des hypogées du Nord-Vaucluse (D'Anna, 1999, p. 156). Ces pointes traduiraient une microrégionalisation d'une production s'insérant dans un vaste ensemble culturel méridional aux influences diverses, dont ferait partie aussi le groupe Rhône-Ouvèze ouvert aux apports languedociens (Morin et al., 2005, p. 352). Comme le mentionnait J. Courtin et G. Sauzade dans leur conclusion sur le poignard d'Orgon, «la découverte d'une pièce Remedello sur le cours inférieur de la Durance, suggère des voies continentales, qui par les cols alpestres, mettent en contact, dès le début du 
III ${ }^{\mathrm{e}}$ millénaire, la plaine du Pô et les régions rhodaniennes » (Courtin, Sauzade, 1975; Morin et al., 2005, p. 356).

Ces différentes découvertes, couplées à celles de nouveaux poignards gravés à Chastel-Arnaud dans la Drôme (ibid.), renvoient, par conséquent, aux rôles des groupes culturels provençaux et de la vallée de la Durance dans la diffusion de la métallurgie du cuivre, depuis les régions transalpines jusque vers le Languedoc. La Provence pourrait s'afficher comme un terrain privilégié pour la reconnaissance des filiations culturelles italiques qui ont dû nécessairement accompagner ces puissants courants de circulation liés à la métallurgie. Ces observations forment aussi un nouvel élément susceptible de préciser les conséquences de tels influx sur l'évolution et la transformation des groupes locaux dans la région provençale.

Les rapports avec l'Italie du Nord sont confirmés, bien au-delà du cadre régional actuel, par la découverte de gravures d'armes analogues au Remedello, jusqu'en Suisse, sur les stèles anthropomorphes des deux importantes nécropoles mégalithiques du Petit Chasseur à Sion dans le Valais (Suisse) et de Saint-Martin-de-Corléans dans la Vallée d'Aoste en Italie (Moinat, Stöckli, 1995; Mezzena, 1998). Quant à la céramique italienne, les niveaux récents de la Chauve-Souris ont livré en parfaite synchronie avec des éléments méridionaux Fontbouisse et des gobelets de style campaniforme rhodano-provençal, de la céramique à décor metopale typique (Vital, 2006). On peut mentionner aussi un vase à métope incisée à la Balme à SollièresSardières en Savoie (Bocquet, 1997). Ces apports d'Italie septentrionale se doublent d'influences d'Italie centrale, révélées par les gobelets à col et carène des ensembles ardéchois de Serre de Boidon à Grospierres et de Beaussement à Chauzon (Montjardin, 1990-1991), et drômois de Pâtis 2 à Montboucher-sur-Jabron (Margarit, Saintot, 2002). Ce type de production est caractéristique de la culture funéraire de Rinaldone (fig. 18 et 19), répandue initialement en Toscane et dans le Latium (Miari, 1998). Elle est, au même titre que le Remedello, intimement liée à la métallurgie du cuivre et notamment à la diffusion des procédés techniques vers le pôle languedocien (Strahm, 2005; Sangmeister, 2005).

\section{Le Campaniforme dans le sud-est de la France}

Le phénomène campaniforme se définit de multiples manières (Guilaine et al., 2004) et actuellement il n'est pas possible de circonscrire clairement son aire d'émergence, malgré les nombreuses hypothèses proposées sur ce sujet depuis les cent dernières années. S'il ne nous semble donc pas utile de revenir sur les origines et les significations données à ce phénomène dans de nombreuses publications (Guilaine, 1967; Besse, 1996; Salanova, 2000; Bailly, 2002; Lemercier, 2004a; Furestier, 2005), il est en revanche nécessaire de garder à l'esprit la question de fond qui a trait aux interactions entre Campaniforme et groupes locaux. En Méditerranée, le processus campaniforme, matérialisé à l'origine par les seuls gobelets maritimes en forme de cloche à décor de bandes ornées pointillées, englobe la péninsule italienne, la Sicile, la Sardaigne, les Baléares, le midi de la France et la plus grande partie de la péninsule Ibérique, à savoir la Catalogne, Valence, l'Andalousie, la Meseta, ainsi que le Portugal centro-méridional (Guilaine, 2004, p. 239). Plusieurs objets se cristallisent secondairement (mais pas systématiquement) autour du gobelet: vases polypodes, pointes de flèches à ailerons et pédoncule dégagé, pendeloques arciformes, poignards à languette en cuivre et pointes de Palmela constituent le set ou package campaniforme (Strahm, 2004). L'interprétation de ce phénomène reste encore en débat. D'abord considéré comme le traceur de migrations européennes massives accompagnées de la diffusion de biens de prestige ou de luxe, les hypothèses convergent à nouveau vers le déplacement de petits groupes de populations porteuses d'objets, mais surtout d'un certain nombre de savoir-faire et de traditions techniques et culturelles. Son émergence, plaquée sur divers fonds néolithiques finaux, contribue à amorcer un rapide désenclavement des frontières régionales, introduisant l'arrivée des premières cultures de l'âge du Bronze (Guilaine, 2004). Très rapidement, les voies de communication et d'échanges ouvertes par les Campaniformes vont jouer dans les deux sens et le sud de la France va voir apparaître de nouvelles traditions d'origine septentrionale (Lemercier, 2004b). Dans le Midi méditerranéen, l'apparition et le développement du Campaniforme interviennent à la fin du Néolithique final, aux alentours de 2500 av. J.-C.

\section{La définition des ensembles}

Dans le sud-est de la France, où plus de 320 sites campaniformes ont été recensés (Lemercier, 2004b, p. 227-229), quatre ensembles ont été définis à partir des formules décoratives céramiques et de leur technique de réalisation (fig. 20) (ibid., Guilaine, 1976b).

Les céramiques décorées de style 1 comprennent les décors cordés, pointillés linéaires, internationaux et mixtes (pointillés et cordés). Elles ne se présentent pas comme une vaisselle complète, mais comme des éléments peu nombreux 


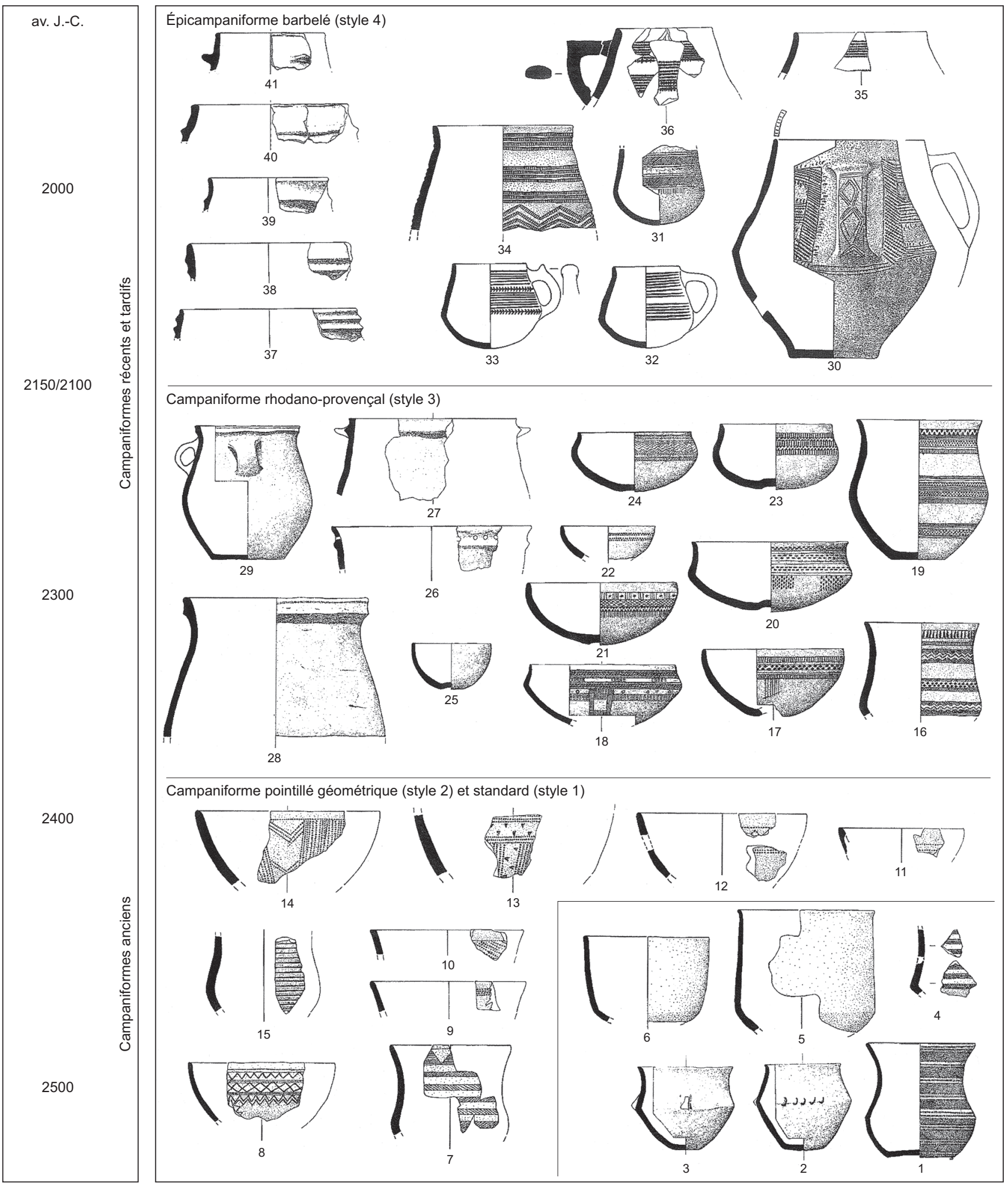

Fig. 20 - Les ensembles céramiques campaniformes et leur chronologie. La céramique décorée et la céramique complémentaire (Campaniforme ancien) ou commune (Campaniforme récent et tardif) (d'après Lemercier, 2004b, p. 228). 
et standardisés (gobelets) qui renvoient au standard défini par L. Salanova (Salanova, 2000 et 2004).

Les céramiques décorées du style 2 regroupent les décors de type pointillé géométrique. Elles présentent une variété de décors et de morphologies en comparaison des vases standard qui y sont souvent associés.

Les céramiques décorées de style 3 montrent des motifs incisés, incisés et estampés, pointillés complexes et définissent, dans le Midi méditerranéen, le groupe régional rhodano-provençal.

Les céramiques décorées du style 4 correspondent exclusivement à des décors incisés et barbelés parfois associés à des motifs estampés. Elles sont marquées à la fois par la tradition campaniforme et par des spécificités qui concernent les morphologies et la technique de décor préfigurant le plein âge du Bronze ancien (Lemercier, 2004b, p. 229). Les comparaisons les plus évidentes s'opèrent avec le style camp de Laure défini dans les Bouches-du-Rhône (Courtin, 1975).

\section{Chronologie du Campaniforme et interactions avec les groupes culturels locaux}

Le Campaniforme évoluerait dans le Néolithique final et le début du Bronze ancien en trois étapes (Lemercier, 2004a; Furestier, 2005). Les styles 1 et 2 seraient contemporains et constituent le premier épisode de 2550-2500 à 2400-2350 av. J.-C., suivi par le style 3 représentant la seconde étape de 2400-2350 à 2150-2100 av. J.-C., puis le style 4 de 2150-2100 à 1900 av. J.-C. Les deux premières phases au moins voient l'interaction des Campaniformes avec les groupes culturels autochtones du Néolithique final de manière certaine.

À l'est du Rhône, les premiers gobelets de styles standard et pointillé géométrique émergent en effet au sein d'assemblages faisant référence au groupe de Fontbouisse et au groupe Rhône-Ouvèze sur des sites généralement situés sur le littoral ou en bordure du Rhône (fig. 21). Les poteries «complémentaires ${ }^{4}$ » des gobelets internationaux sont donc des productions indigènes du Néolithique final. À la Fare à Forcalquier (Alpes-de-Haute-Provence) par exemple

4. La céramique complémentaire se définit comme la céramique qui est associée aux éléments du set, et par une céramique domestique de tradition locale (Rhône-Ouvèze ou Fontbouisse dans le cadre de notre zone d'études) accompagnant le gobelet campaniforme décoré. Elle est différente de la céramique «commune» qui correspond à la céramique domestique du groupe campaniforme rhodano-provençal avec des formes caractéristiques (Besse, 1996 et 2004; Strahm, 2004). ou encore aux Calades à Orgon et à Escanin aux Baux-deProvence (Bouches-du-Rhône), les productions associées aux gobelets internationaux ou à leurs proches dérivés entrent dans la panoplie des céramiques locales (Lemercier, 2004b). Ce faisant, le lien entre le gobelet maritime et les morphologies «autochtones» précampaniformes a été observé par ailleurs dans la péninsule Ibérique, autant sur son versant méditerranéen que sur son versant atlantique. Une situation identique vaut aussi pour la Sicile (Guilaine, 2004, p. 242). On retient par conséquent l'impression que lorsque les premiers gobelets campaniformes (styles 1 et 2) se propagent en domaine méditerranéen, ils se diffusent pleinement dans les cultures en place du Néolithique final. L'existence d'ensembles clos attestant d'une stricte synchronie entre groupes locaux et premiers campaniformes argumente dans ce sens, comme dans la sépulture S14 de la Fare associant un vase à décor mixte (peigne et cordelette) et deux gobelets Rhône-Ouvèze (Lemercier, Furestier et al., 2004). L'observation macroscopique des céramiques suffit, elle aussi, à affirmer l'unité des productions campaniformes et Rhône-Ouvèze/Fontbouisse sur des sites comme la Balance à Avignon et le Fortin du Saut à Châteauneuf-les-Martigues (Bouches-du-Rhône). Des analyses pétrographiques réalisées par F. Convertini ont enfin confirmé cette unité pour certains sites comme les Barres à Eyguières (Bouches-du-Rhône), et la plaine de la Vaunage dans le Gard, où des vases Rhône-Ouvèze ou Fontbouisse ont été façonnés selon des techniques campaniformes tels l'emploi de la chamotte dans les vases locaux ou, inversement, l'emploi de la calcite propre au Rhône-Ouvèze et au Fontbouisse pour des récipients campaniformes (Convertini, 1996 et 1998). L'existence de transferts stylistiques valide également l'hypothèse de la synchronie des ensembles. Certains cas d'objets mixtes sont bien connus comme le petit vase à décor Fontbouisse à fond ombiliqué campaniforme de la place du Palais à Avignon (Courtin, 1974; Sauzade, 1983). D’autres ont été mis au jour récemment au Fortin du Saut à Châteauneuf-les-Martigues dans les Bouches-du-Rhône, où un gobelet de typologie campaniforme présente un décor Fontbouisse (Furestier et al., 2007). Le constat de transfert stylistique et technique entre les entités locales et campaniformes abonde aussi hors de la Provence. Aux exemples de panneaux horizontaux à décors incisés verticaux que l'on retrouve dans la grotte de la Chauve-Souris à Donzère dans la Drôme (Vital, 2006), s'ajoute désormais le gobelet campaniforme à cannelures du mas de Vignole IV à Nîmes dans le Gard (Convertini et al., 2004). 


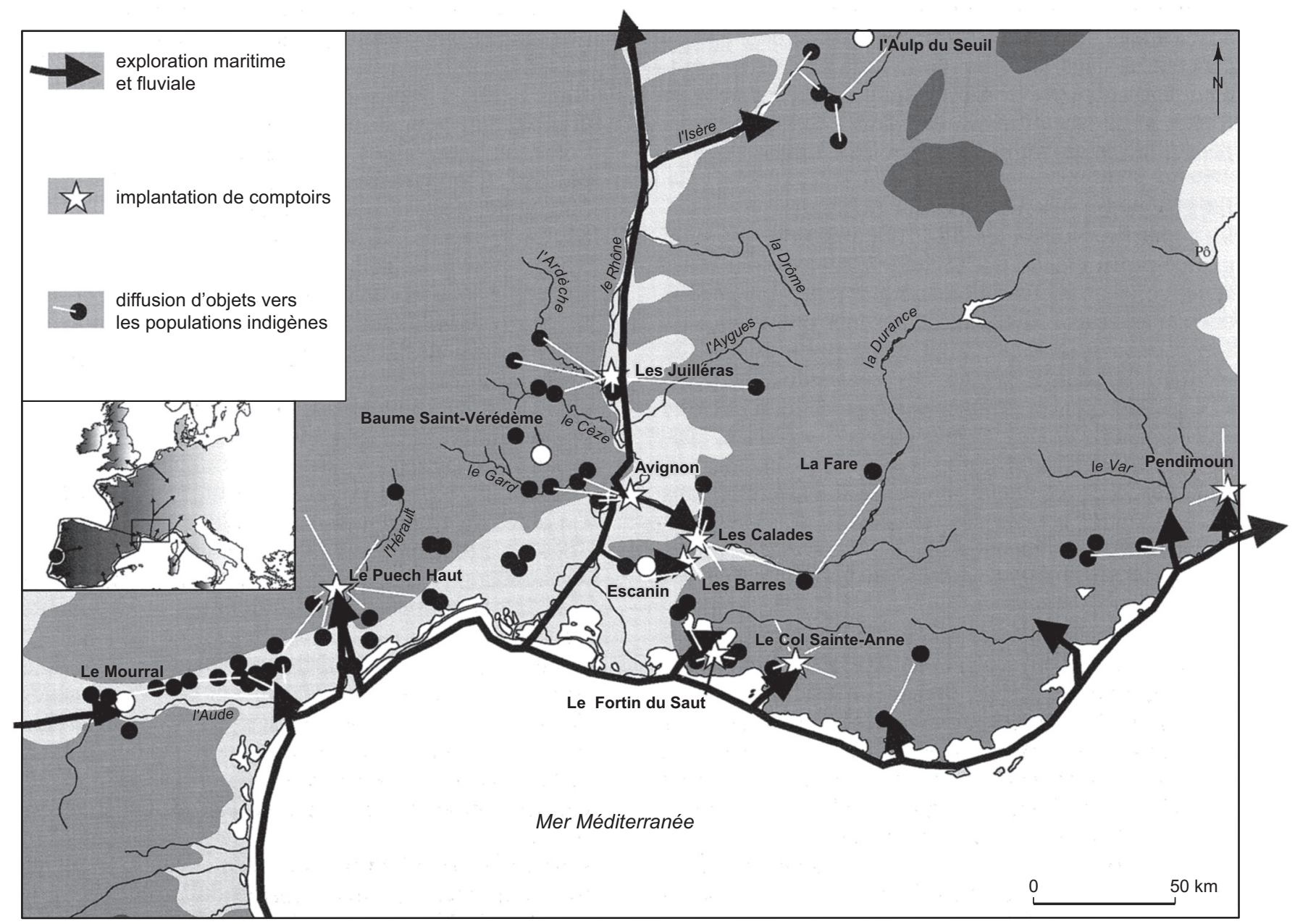

Fig. 21 - Interprétation du phénomène campaniforme en France méditerranéenne. Carte des explorations, des implantations et des diffusions du Campaniforme ancien (d'après Lemercier, 2004b, p. 231).

À l'ouest du Rhône, pas plus d'une trentaine de sites sont concernés par l'intégration du Campaniforme ancien: style standard et pointillé géométrique (Gutherz, 1988; Roger, 1988 et 1995; Lemercier, 2004a). Il est remarquablement absent du Languedoc oriental sur l'aire d'extension du Fontbouisse, malgré la présence sporadique de quelques gobelets.

Dans la seconde phase, les groupes campaniformes régionaux (rhodano-provençal, céramique de style 3) tendent à remplacer les cultures locales du Néolithique final, par acculturation amorcée dès les premiers temps de la présence campaniforme (Lemercier, 2007, p. 496). En Provence, le prolongement du groupe Rhône-Ouvèze jusqu'à la période de plein développement du groupe rhodano-provençal est attestée par quelques sites, comme celui de la Bastide Blanche à Peyrolles-en-Provence dans les
Bouches-du-Rhône (Lemercier et al., 2006). Le Languedoc oriental, initialement non concerné par la diffusion du Campaniforme dans sa phase ancienne, est en revanche gagné par les implantations campaniformes régionales. D'importantes relations sont par ailleurs maintenues avec le monde ibérique et plus particulièrement avec le centre et le nord-est de l'Espagne (Lemercier, 2004b).

Les groupes campaniformes sont pleinement autonomes dans la dernière étape, pendant laquelle les cultures locales auraient totalement disparu. Les céramiques campaniformes du groupe barbelé (style 4) associent à une céramique domestique spécifique une culture matérielle complète. De nouveaux influx d'origine septentrionale (culture du Rhône) montrent cette fois l'importance des échanges ouverts par les premiers campaniformes le long de la vallée du Rhône (ibid., p. 236). 


\section{Évolution et transformations des groupes locaux au contact du Campaniforme}

Ce schéma global de mise en place et d'évolution du Campaniforme dans le sud-est de la France ouvre plusieurs perspectives de recherches relatives au rapport qu'entretiennent les cultures provençales locales et le Campaniforme. Un point de problématique peut être résumé ainsi: qu'en est-il de l'autonomie du Campaniforme par rapport aux groupes culturels synchrones de la fin du Néolithique, Rhône-Ouvèze et apparentés Fontbouisse ? Le phénomène campaniforme, qui touche le sud et l'ouest de la France, s'insérerait dans les groupes allogènes (style 1 et 2) avant d'être autonome (style 3 et 4 ). Le rôle des «substrats» locaux dans le fonctionnement du système campaniforme est sans doute appréciable, dans la mesure où la céramique complémentaire est possiblement porteuse d'informations sur les origines du phénomène, ses modalités et les causes de sa diffusion (Lemercier, 2004b, p. 237). Les auteurs, qui se sont déjà penchés sur la genèse du processus campaniforme, ont d'ailleurs tenté à plusieurs reprises de retrouver dans certains substrats autochtones des éléments céramiques morphologiques et/ou décoratifs susceptibles d'avoir eu un retentissement sur l'apparition de la culture des gobelets (Guilaine, 2004, p. 247).

Par effet contraire, les cas de mixités stylistiques et de parentés dans les techniques de façonnage constatés entre les groupes autochtones Fontbouisse et Rhône-Ouvèze et les ensembles Campaniforme ancien permettent aussi de formuler l'hypothèse selon laquelle le Campaniforme peut constituer une composante de premier plan dans l'évolution des groupes culturels provençaux.

$\mathrm{Au} \mathrm{XXV}^{\mathrm{e}}$ s. av. J.-C., le phénomène campaniforme s'accompagne en effet d'innovations considérables susceptibles d'avoir eu des conséquences sur la structuration des sociétés in situ. Les petits groupes de populations campaniformes dotés de nouveaux savoir-faire et de traditions techniques et culturelles spécifiques se déplaceraient dans la région et entérineraient, en domaine méditerranéen, de nouveaux axes d'échanges et de circulation à longues distances. Si les cultures italiennes tel le Remedello sont déjà des acteurs dans la diffusion de la métallurgie au-delà des Alpes et que la métallurgie sur le territoire français est d'abord précampaniforme (Guilaine et al., 2009, p. 165), le Campaniforme reste indissociable du déploiement même de la métallurgie du sud-est par la propagation des pointes de Palmela et des poignards à languette. Ces deux entités culturelles, le Campaniforme et le Remedello, détentrices des pratiques métallurgiques, sont d'ailleurs directement en relation en Provence orientale, où l'apparition des premiers campaniformes sur le site de l'abri de Pendimoun (Castellar, Alpes-Maritimes) est accompagnée d'influences d'origine italique avec la présence de céramique à décor metopale (Binder, 2003). Dans la Drôme, les niveaux récents de la Chauve-Souris à Donzère ont livré en parfaite synchronie avec des éléments méridionaux Fontbouisse et des gobelets de style campaniforme rhodano-provençal, de la céramique à décor metopale typique (Vital, 2006). Aussi, vers les XXV-XXIV s. av. J.-C., la Provence polarise sans commune mesure des influences occidentales et orientales fortes, issues de deux directions opposées, dont la prégnance sur le fonctionnement et la transformation des groupes culturels provençaux reste à comprendre.

\section{DISPARITION OU SURVIVANGE DES GROUPES PROVENÇAUX AU PASSAGE AU BRONZE ANGIEN}

La question de la persistance de traditions culturelles du Néolithique final au passage vers le Bronze ancien doit être également posée pour la région méridionale. Comme d'autres il y a trente ans (Guilaine, 1972; Roudil, 1972; Bill, 1973), J. Vital et X. Gutherz ont récemment fait la synthèse sur cet aspect. Parmi les points importants développés, on soulignera la reconnaissance du poids campaniforme et la part qu'il convient d'accorder au substrat régional dans la genèse des cultures du Bronze ancien pour le sud-est de la France (Gutherz, 1995; Vital, 1996, 2004 et 2005a) : en particulier dans la civilisation du Rhône issue du prolongement de la culture Cordé et Unetice vers 2100 av. J.-C. (Bailloud, 1966; Gallay, 1976 et 1996; Vital, 2006).

Dans les hypothèses actuelles, les composantes autochtones du Campaniforme et plus largement du Néolithique final semblent cependant secondaires. S'il existe un héritage du fonds céramique campaniforme et local au Bronze ancien en particulier dans la moyenne vallée du Rhône, l'origine de ce dernier déborderait largement le cadre suprarégional pour renvoyer à l'Europe centrale. Il semble en effet que le Bronze ancien puisse à la fois relever des groupes tardifs du Campaniforme, du Cordé oriental et du Bronze ancien plus occidental (plateau Suisse), dont les relations chronologiques demandent encore à être affinées (Vital, 2004, p. 273). Quelques séquences stratigraphiques de référence permettent néanmoins d'illustrer le passage du Néolithique final à l'âge du Bronze ancien dans le secteur médio-rhodanien: la grotte de la Chauve-Souris à Donzère, l'établissement de plein air du Serre 1 à Roynac dans la Drôme, ainsi que plusieurs sites lyonnais de la plaine 
de Vaise, dont les plus significatifs sont ceux de Gorge de Loup et du boulevard périphérique nord (Vital, 2002, 2005a, 2006 et 2008; Durand, 2002). Sur ces gisements, la tendance actuelle serait en faveur d'une évolution ultime du Campaniforme rhodano-provençal avant les faciès barbelés du Bronze ancien, dans un court hiatus estimé de 2200 à 2100 av. J.-C. Ces sites rompraient alors avec les schémas d'une succession directe Campaniforme rhodano-provençal/Bronze ancien barbelé. À côté de petits gobelets carénés, de jattes et d'écuelles à décors de bandes incisées et/ou peignées de tradition campaniforme, voisinent en effet de plus gros récipients d'affinités plus largement Néolithique final et des jarres à cordon de facture Bronze ancien.

Plus au sud, côté rive droite du Rhône, les cas longtemps mentionnés dans les travaux de X. Gutherz (1995) ne sont aujourd'hui plus d'actualité. Ils laissaient notamment supposer la possibilité d'un prolongement des styles au Bronze ancien, non plus à partir du substrat campaniforme comme en domaine rhodanien, mais à partir des complexes régionaux, tel le Fontbouisse. Dans tous les cas, la combinaison entre des éléments Fontbouisse et ceux du Bronze ancien s'est révélée être le fruit de télescopages sédimentaires ou d'héritages résultant des conditions taphonomiques spécifiques aux gisements. Tel est le cas dans les grottes de Peyroches II (Saint-Alban-Auriolles, Ardèche), dans la grotte Suspendue et dans celles de Pâques 2 à Collias ainsi qu'à la grotte des Pins à Aubais dans le Gard (Dufraigne et al., 2003) et dans la couche 3 du site de Triple (Beaucaire, Gard). Pour chacun des gisements, il semble qu'il faille revenir sur l'hypothèse graduelle d'une transition du Campaniforme rhodano-provençal au Bronze ancien (Vital, 2005a, p. 19). J. Vital reconnaît cependant en Languedoc, dans les niveaux les plus récents du site éponyme du Fontbouisse et au Rocher des Causses à Claret dans l'Hérault, du mobilier comparable à ceux attribués à cette interface de transition dans la zone rhodanienne.

À l'est du Rhône, la documentation est particulièrement indigente. Il faut admettre que l'absence de site qui précèderait les plus anciennes manifestations du Bronze ancien ne permet pas de démêler cet intervalle de temps compris entre la disparition des groupes locaux Rhône-Ouvèze et Nord-Vaucluse, le développement du Campaniforme rhodano-provençal et la genèse du Bronze ancien. Tout au plus, J. Vital n'exclut pas que la série de vases de l'abri de Fraischamp 2 à Roques-sur-Pernes dans le Vaucluse (Bill, 1973) relève d'une phase tardive du Campaniforme à rapprocher là encore des niveaux Bronze ancien du boulevard périphérique nord de Lyon (Vital, 2005a, p. 19).
Dès lors, le nœud du problème ne peut être éliminé, mais le débat se place aussi ailleurs. D’un point de vue plus global, la réflexion tourne autour de la place à donner aux nouveaux processus qui caractérisent l'âge du Bronze ancien. Pour certains archéologues, dès l'introduction de la métallurgie, tout comme au passage du Néolithique final au Bronze ancien, des mutations sociales importantes s'opèrent. Elles sont générées par cette innovation technologique, qui induit le développement des échanges, l'apparition de classes dirigeantes, des élites, des inégalités sociales, la diversification des compétences, la spécialisation artisanale, l'accumulation de biens socialement valorisés, le développement de l'ostentatoire dans les contextes architecturaux... (Ambert, 1990-1991; Guilaine, 1994, p. 123-136 et 2007; Carozza, 2000 et 2002). D'autres, en revanche, n'accréditent pas l'hypothèse du rôle majeur de la métallurgie dans les changements sociaux que l'on croit percevoir à la fin du Néolithique dans le sud de la France. Il leur semble que les signes d'une telle transformation préexistent à la métallurgie (Strahm, 2005, p. 35; Gutherz et al., 2005, p. 128). Par exemple, dès le milieu du IVe millénaire, à des dates antérieures à la métallurgie, de grandes sépultures mégalithiques sont considérées comme le signe d'un ancrage territorial et, indirectement, d'une société hiérarchisée. Parallèlement, l'apparition des statues menhirs traduit peut-être un culte du grand ancêtre ou de la divinité propre à un groupe lignager ou clanique. Il pourrait vraisemblablement s'agir d'instruments iconographiques susceptibles de répandre l'image du pouvoir (Gutherz et al., 2005, p. 128).

Si l'on adopte ce dernier point de vue, particulièrement défendu par C. Strahm, les derniers groupes du Néolithique final et les premières cultures du Bronze ancien ne diffèrent pas totalement et s'inscrivent même dans des univers socioéconomiques très proches (Strahm, 1996 et 2005). En effet, avec les cultures Néolithique final, la métallurgie, bien qu'en plein essor, reste de type chalcolithique, c'est-à-dire exploitée par de petits groupes humains transformant le minerai dans les environnements immédiats pour une consommation domestique (ibid.). Au début du Bronze ancien, rien n'indique non plus qu'une organisation centrale aux mains de l'élite et de l'autorité se soit imposée (période dénommée Chalkolithikum au sens phaséologique de C. Strahm, 1982), à la différence du plein âge du Bronze (Metallikum). Il faut en réalité attendre le Bronze final pour constater l'avènement en France d'un nouvel ordre social qui résulte d'une évolution déclenchée par l'introduction plus massive de la métallurgie. 
En ce sens, le passage à l'âge du Bronze, tout comme le Bronze ancien lui-même, apparaissent là comme des stades historicoculturels potentiellement néolithiques. Comme le soulèvent C. Strahm et J. Vital, la permanence, dans le champ du symbolique (modalités architecturales et funéraires), comme dans la culture matérielle (parures et céramique), de liens entre les cultures du Néolithique final, du Campaniforme et du début du Bronze ancien repose avec plus d'acuité la notion réelle de rupture associée à la transition entre ces deux époques (Vital, 2004, p. 273 et 2005a, p. 32; Strahm, 2005).

\section{QUELLES ORIENTATIONS POUR LA RÉSOLUTION DES PROBLÈMES SOULEVÉS ?}

\section{LE TEMPS ET L'ESPACE}

Face à l'ampleur des questions ouvertes, le premier impératif est de circonscrire l'étendue chronologique du champ d'étude pour concentrer les enquêtes de manière à les rendre non seulement réalisables, mais aussi cohérentes et pertinentes. Même s'il n'est pas utile d'intégrer directement les phases antérieures et postérieures au Néolithique final stricto sensu dans le sud-est de la France, de 3400 à 2400 av. J.-C., les deux stades de franchissement entre Néolithique récent et Néolithique final d'une part et Néolithique final et âge du Bronze ancien d'autre part doivent être abordés. Le choix de considérer le début et l'extrême fin du Néolithique est motivé aussi par le constat que la notion de rupture associée aux transitions entre les différentes époques apparaît de plus en plus désuète. Il est, de plus, intéressant de considérer une problématique sur une longue durée afin d'offrir un cadre pour apprécier les changements et les évolutions relatives aux sociétés néolithiques, ici des systèmes postchasséens aux systèmes Bronze ancien.

Sur l'ensemble de cette séquence comprenant la deuxième moitié du $\mathrm{IV}^{\mathrm{e}}$ millénaire et le III $^{\mathrm{e}}$ millénaire av. J.-C., quelques hiatus sont toutefois à relever dans la construction de la chronologie absolue par le radiocarbone. La courbe de calibration est particulièrement bouleversée pour la période considérée et l'irrégularité de la production de radiocarbone dans l'atmosphère engendre une forte différence entre les millénaires en radiocarbone et les millénaires en années solaires. Cette courbe présente donc plusieurs plateaux, pour lesquels on obtient généralement un large intervalle de temps après calibration. La réduction de la marge statistique n'améliore en aucune façon la précision de l'intervalle (Oberlin, 2003). Pour la période considérée, la résolution chronométrique est donc limitée, ce qui brouille à terme les interprétations chronologiques (Pétrequin et al., 1998; Bailly, Salanova, 1999), alors que les enjeux que représente la chronométrie dans la compréhension des processus de mise en place, de successions et de chevauchements entre les groupes du Néolithique final sont fondamentaux. Dans l'état actuel des données, le gradient de finesse dans la lecture du cadre chronométrique de la période est aussi décroissant selon un axe nord-sud, depuis le plateau Suisse, l'Italie nord-occidentale, jusqu'au Languedoc et la Provence (Vital, 2005a). Notre zone d'études occupe ainsi une position très lacunaire à la périphérie de secteurs où la chronologie est en revanche très élaborée (plateau Suisse), voire maîtrisée (Languedoc). La construction de la grille chronoculturelle provençale peut donc s'effectuer en parallèle avec les données de ces régions adjacentes.

$\mathrm{Au}$ Néolithique final, l'entité géographique provençale trouve grosso modo une cohérence en termes de découpages culturels et techniques: un faisceau de facteurs illustre en effet les particularités inhérentes à cette sphère géoculturelle, au premier rang desquelles la discrétion des traces de production et de consommation d'objets métallurgiques dans la région, identifiées pourtant en abondance à l'ouest du couloir rhodanien et à l'est des Alpes dans les groupes languedociens et italiens. Plus tard, au XXV $\mathrm{XV}^{\mathrm{e}}$. av. J.-C., la Provence se distingue également car elle est petit à petit démantelée au profit des intrusions campaniformes, tandis qu'en Languedoc l'ancrage du peuplement local prévaut, en même temps que dans le bassin du Rhône moyen, la culture Cordé semble s'imposer au détriment des groupes autochtones. Il n'en demeure pas moins que, dans le bilan dressé au préalable, nous avons pu constater que l'intégration d'un vaste espace géographique permettait de cerner la réalité d'une période complexe dans laquelle les mécanismes d'imbrication sont patents. Suivant cette logique, les travaux doivent être axés sur un large sud-est, dépassant le cadre régional, tout en gardant néanmoins la possibilité d'élargir ou de réduire l'assiette par rapport aux procédés d'analyse convoqués et/ou aux questionnements posés. De cette façon aussi, l'aire intègre une géographie contrastée et riche, depuis la vallée du Rhône ouverte à l'ouest et à l'est sur de grandes plaines et, au niveau de son embouchure, sur des zones basses, jusqu'aux sommets alpins, en passant par des secteurs très variés de collines, de plateaux et de massifs côtiers. L'ensemble de cette région est, de plus, parcouru d'importantes voies de pénétration fluviale et terrestre, à 
commencer par les vallées du Rhône et de la Durance qui permettent la circulation et les échanges.

Le champ chronologique et géographique ainsi envisagé ouvre des perspectives d'étude intéressantes. Il offre une étendue et une diversité permettant d'organiser les observations selon plusieurs abords: diachronie et synchronie d'une part, micro et macrorégional d'autre part. La maîtrise de la diachronie et de la synchronie, ainsi que le va-et-vient constant entre différentes échelles d'analyse géographique doivent permettre d'étudier l'évolution conjointe, le rythme, l'ordre d'apparition et de disparition des diverses composantes culturelles de la fin du Néolithique. Il s'agit aussi de démontrer les mécanismes de structuration des entités, en analysant leurs variabilités et leurs récurrences dans leurs manifestations culturelles.

\section{TYPOLOGIE ET STYLISTIQUE CÉRAMIQUE: L'APPROCHE DESGRIPTIVE DÉTAILLÉE COMME MOYEN D'ACCÈS À L'INFORMATION CULTURELLE}

\section{POURQUOI LA GÉRAMIQUE ?}

Dans le domaine de la Préhistoire récente, les données issues de la culture matérielle sont à la base même de l'identification et de la définition des cultures archéologiques. Celles-ci sont composées d'un ensemble de traits et de vecteurs identitaires similaires au sein d'un espace géographique cohérent, pour un temps donné. Le style des formes et des décors céramiques en particulier demeure aujourd'hui le traceur principal, sollicité par l'archéologue, dans la définition des groupes culturels. Cette équivalence entre style céramique et groupe culturel admet aussi que les décors et les formes céramiques reflètent les modalités de fonctionnement des cultures. Ils permettent de suivre notamment leur mise en place, leur évolution, leurs transformations et leurs interactions.

Parvenir à analyser ces mécanismes de sociétés au Néolithique final dans le sud de la France est un enjeu majeur, qui passe naturellement par la compréhension de la structuration stylistique des productions céramiques, sans mésestimer toutefois les approximations que ce parti pris induit.

Tout d'abord, l'équation céramique = groupe ouvre la porte à un compartimentage et à un morcellement excessif et stérilisant des processus culturels, enfermés dans des cadres rigides et fixés, ne tenant pas compte des nuances. La réalité peut être bien plus complexe, ne serait-ce qu'en raison de l'extrême variété des situations. Ainsi, telle forme ou tel décor peut varier au sein d'une même culture supposée; à l'inverse, deux cultures distinctes peuvent avoir, localement ou de façon plus large, les mêmes traditions dans le système ornemental ou le répertoire morphologique (Van Berg, 1983, p. 106; Dietler, Herbich, 1994).

On sait par ailleurs que la céramique n'a pas la capacité de retranscrire la totalité des phénomènes historico-culturels. Le concept de culture est d'abord un concept d'observateur et ensuite un outil, mais rien de plus. Les cultures, les groupes, les entités sont fondamentalement des unités de partage de traits culturels particuliers, matérialisés, mais pas nécessairement superposables aux cultures émiques. Les divers taxons spatio-chronologiques de l'histoire culturelle (Rhône-Ouvèze, Fontbouisse, Couronnien...) subissent malheureusement parfois un glissement sémantique et finissent par être entendus plus ou moins comme des groupes culturels au sens ethnologique du terme (Tremblay, 1999, p. 5). Si dans les travaux en Préhistoire, des entités pour lesquelles sont recherchés les mécanismes d'imbrication et les dynamiques de formation et d'évolution peuvent être isolées, il ne doit pas s'agir pour autant de mettre en lumière des processus de modification ethnique ou identitaire. Nous n'isolons pas non plus des formations sociales et nous tentons encore moins de repérer des mécanismes de peuplement (Barth, 1969; Shennan, 1990). La culture matérielle génère seulement des entités typologiques et stylistiques cohérentes, margées et en évolution. Elle circonscrit des espaces de partage de traits culturels exprimés dans la matière; éventuellement, comme l'ont montré les études de A. Gallay ou de De Ceuninck, la répartition spatiale et chronologique de certaines formes et décors peut correspondre à des sphères d'endogamie ${ }^{5}$ (Gallay, Ceuninck, 1998, p. 29). Pour autant, les différents indices de la culture matérielle ne peuvent pas être rapportés à des entités, "traduites en ethnographie, en identité ou articulées en histoire et en processus» (Clermont, 1999, p. 71). Le succès du document céramique dans la construction des schémas chronoculturels ne doit pas faire oublier certaines caractéristiques propres à ce genre de production, qu'il serait nécessaire de maîtriser pour garantir la validation des hypothèses. Il en est ainsi des informations liées aux traditions et aux modes, à la spécification de la production, aux vecteurs de sa diffusion, aux différentes

5. Endogamie: «Règle obligeant un individu à choisir son conjoint à l'intérieur du groupe culturel auquel il appartient, quel que soit le principe définissant ce groupe (parenté, classe, caste, territoire, etc.) » (Dupuy, 2004, p. 183). 
formes d'utilisation, aux exigences de fonctionnement et aux modalités d'abandon (Vital, 2005b, p. 28).

Enfin, à elle seule, la céramique ne peut être considérée comme l'unique et le plus pertinent des descripteurs archéologiques (Boissinot, 1998). On présuppose d'ailleurs que chaque type d'artefact peut suivre une évolution spécifique comme le suggèrent certains exemples archéologiques (Voruz, 1984; Pétrequin et al., 1987-1988, p. 4) et maints cas ethnographiques (Pétrequin et al., 1993). L'autonomie des mobiliers de la culture matérielle (céramique, outillages lithique et osseux, faune mammalienne, parure...) existe dans leur répartition spatio-temporelle (ibid.; Voruz et al., 1995, p. 395). C'est ainsi, par exemple, que M. Honegger, comme d'autres avant lui, a mis en évidence que l'évolution des composantes culturelles de la céramique et de l'outillage taillé des Néolithiques moyen et final de Suisse pouvait fonctionner de manière totalement indépendante, délivrant des lectures de la période différentes en fonction du type d'artefact (Honegger, 2001, p. 196).

Ceci étant, de nombreuses études ont démontré l'importance des styles céramiques dans la recherche des relations entre production matérielle et faits culturels (Conkey, 1990; Ceuninck, 1994; Demoule, 1994; Crits, 1994). «La variabilité typologique des corpus ainsi que l'analyse de la structure des décors sont de bons indices de la dynamique des systèmes stylistiques. » «Il est possible [...] d'avancer que le style céramique remplit une fonction au sein de la société et participe à l'élaboration de stratégies identitaires » (Giligny, 1994, p. 377-378).

\section{DES SÉRIES QUANTITATIVEMENT IMPORTANTES ET GHRONOLOGIQUEMENT BIEN CALÉES DANS DES ESPACES ENVISAGÉS À LARGE ÉCHELLE}

\section{Concevoir les groupes à travers une vision polythétique}

Pour le sud de la France, les assemblages culturels sont encore perçus comme des civilisations monothétiques (Clarke, 1978, p. 30-41), sorte de blocs culturels variant toujours ensemble et dans le même sens. Les groupes, Couronnien, Fraischamps, Rhône-Ouvèze, Nord-Vaucluse, ont été conçus seulement sur le résultat acquis par l'observation empirique d'une petite quantité de mobilier céramique.

Le bilan réalisé en liminaire montre que les données actuelles révèlent des réseaux complexes et foisonnants régissant les sociétés. Aujourd'hui, les modèles polythétiques de D.-L. Clarke (1978, p. 245-298) semblent être une solution

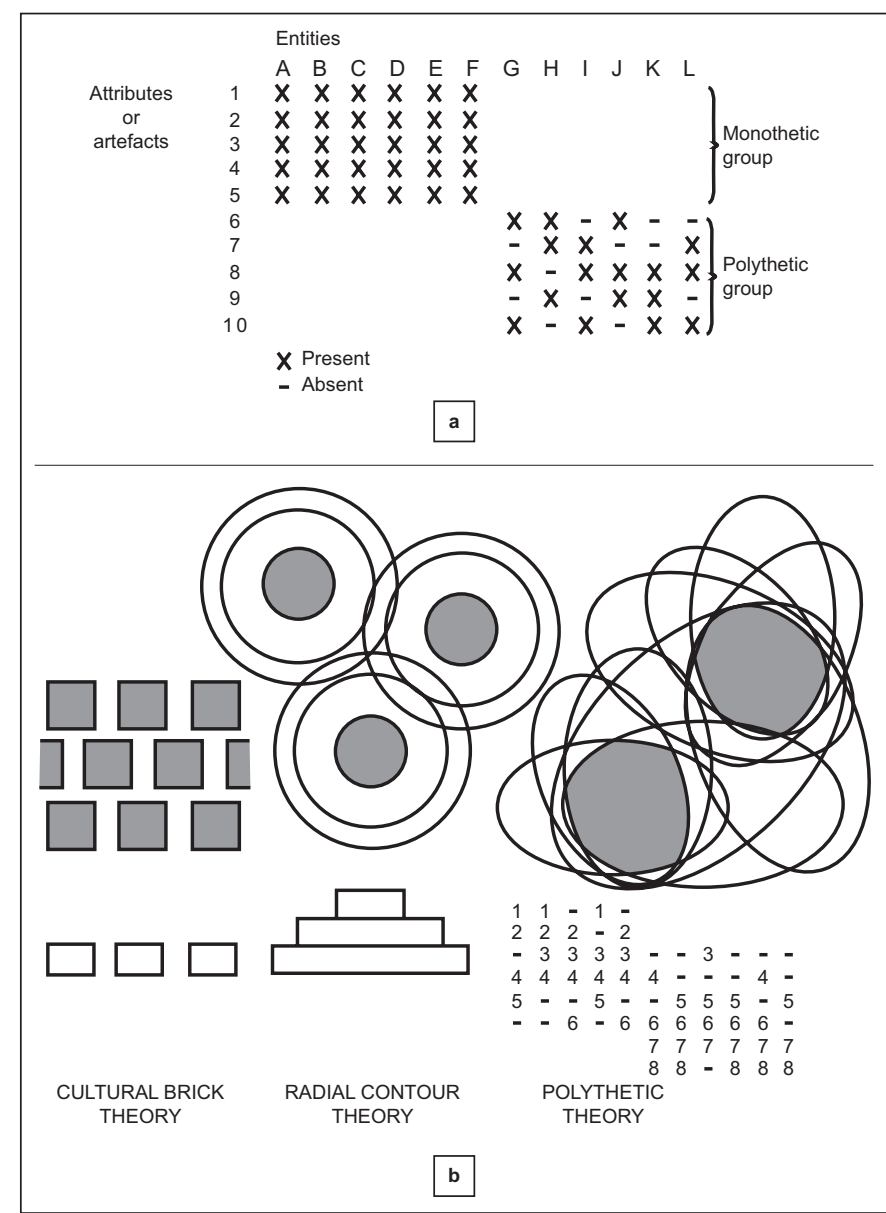

Fig. 22 - Les modèles polythétiques selon D.-L. Clarke (d'après D.-L. Clarke, 1978, p. 36, fig. 3 et p. 264, fig. 67).

pour tenir compte de façon plus juste des réalités culturelles en travaillant sur des groupes identifiés par des types clefs (artefacts spécifiques à un ensemble géographique défini), des types exclusifs (témoins de l'originalité de certaines entités à l'intérieur du groupe étudié) et des types non essentiels (indiquant des phénomènes de continuité et d'intercommunication avec d'autres groupes et d'autres régions (Pétrequin, 1997) (fig. 22). Ainsi considérés, les ensembles sont conçus comme des entités disposant à la fois de leurs caractéristiques propres, mais aussi d'autant d'éléments traduisant des relations avec les entités qui les entourent, leur succèdent ou les précèdent. Les groupes sont donc pensés comme des entités mouvantes, en relation et en réseaux. Toutefois, ces modèles ne prennent de sens qu'à partir d'études détaillées et chiffrées très exhaustives, pour lesquelles on conçoit de partir du singulier pour reconstituer des ensembles à valeur plus large. Ils n'ont 
donc d'efficacité que si l'on s'en sert pour étudier des séries livrant une information abondante et placées dans un contexte chronologique fiable, des sortes de «vitrines statistiques reflétant une production collective dans une forme d'instantané culturel»(Clermont, 1999, p. 73). Ce principe s'applique de manière particulièrement opérante dans le cadre du Néolithique lacustre, connu pour ses gisements d'importance fournissant de grandes séries d'artefacts et pour lesquels la maîtrise de l'écoulement du temps s'opère à une résolution de 25-50 ans grâce aux datations dendrochronologiques.

Le degré d'applicabilité pour le sud-est de la France est certes moindre que sur les établissements lacustres, d'abord parce que les sites sont moins bien conservés et ensuite parce que nous ne pouvons, étant donné l'avancement des recherches, nous concentrer que sur la céramique aux dépens des autres témoins archéologiques. L'échelle du temps est également considérée à une résolution nettement moins précise que sur les habitats en milieu humide. Nous proposons une approche qui se veut néanmoins exhaustive. En insistant sur les caractéristiques propres à chaque site envisagé par le biais d'un examen détaillé de séries céramiques bien calées, on espère œuvrer à petite échelle géographique pour construire des séquences d'évolution régionale et ainsi tenter des corrélations à des échelles de complexité croissante, qu'il s'agisse de la couverture spatiale ou chronologique et de la variabilité des productions (Vital, 2005b, p. 26).

\section{Sortir du localisme culturel}

Le fonctionnement des sociétés de la fin du Néolithique dans le sud-est de la France n'a d'intérêt que perçu à large échelle. Cela permet de saisir dans son ensemble une région richement documentée, marquée par de forts contrastes géographiques et culturels. Plus que les particularismes, ce sont les phénomènes culturels dans leur globalité qui nous interpellent (Guilaine, 1994). Eux seuls permettent d'établir des synchronismes, des corrélations sur des territoires suffisamment grands pour mettre en lumière des faits à valeur générale dans une chronologie prédéfinie. Pour ce faire, toutes les questions de mise en place des groupes, d'évolutions, d'interactions, de successions ne peuvent aujourd'hui être abordées qu'en sortant du localisme culturel. C'est la raison pour laquelle, il est impératif de ne pas se limiter au traitement d'un seul groupe défini. Plus encore, la confrontation des séries céramiques provenant de sites archéologiques divers attribués initialement à plusieurs cultures distinctes (Fraischamp, Couronnien, RhôneOuvèze et Nord-Vaucluse pour la Provence, Fontbouisse pour le Languedoc et la Drôme, Néolithique final dauphinois pour l'Isère, des sites à Campaniforme et d'autres à Remedello et Rinaldone) doit s'accomplir en faisant obligatoirement l'économie de l'attribution culturelle admise. Celle-ci ne doit constituer qu'un «filtre» et doit être employée dans son acceptation la plus neutre. Le but est d'examiner et d'interpréter de bonnes séries céramiques au sein d'une plage chronologique et dans un espace géographique défini et vaste, sans être bloqué par les dénominations chronoculturelles a priori. De cette façon, l'intérêt est davantage porté sur la détermination des dynamiques évolutives des productions au sens large. Une fois la totalité des aspects typologiques et stylistiques enregistrée à partir de séries céramiques majeures bien circonscrites dans le temps et l'espace et étudiées de façon précise, il est possible de mieux connaître les productions céramiques dans leur variabilité, les dynamiques d'échanges et de transmission. Cela permet de concevoir d'autant plus fidèlement le pluralisme des sociétés néolithiques.

\section{UNE APPROCHE MORPHO-TYPOLOGIQUE ET STYLISTIQUE}

Depuis plusieurs années, de nouvelles voies sont explorées et abordent la technologie culturelle par l'analyse des savoir-faire et la caractérisation physico-chimique des productions céramiques. Malgré cela, un examen caractérisant les cortèges céramiques d'un point de vue exclusivement typologique est d'abord nécessaire. Celui-ci demeure un préambule avant l'exploitation plus large des potentiels heuristiques de la technologie céramique dans le cadre d'études spécifiques. La gestion du territoire, les secteurs d'approvisionnement, les choix et les évolutions techniques, la question des fonctions, les rapports et identités culturels sont en effet parmi les thèmes aujourd'hui abordés grâce aux études technologiques pétrographiques (Binder, Échallier, 1991; Échallier, Thiercelin, 1991; Convertini, 1996 et 1998; Di Pierro et al., 2005; Rodot et al., 2005; Martineau et al., 2007), par analyses d'images (Arnal et al., 1991; Durrenmath, 1998, 2001 et 2005) expérimentales et ethnoarchéologiques (Binder, Courtin, 1994; LivingstoneSmith et al., 2005). Certains chercheurs même n'hésitent pas, et c'est ce que O.-P. Gosselain met particulièrement en valeur, à évoquer les notions de «style technique»en admettant que chaque assemblage d'objets ou chaque objet est susceptible d'incorporer dans ses processus techniques 
l'expression des multiples facettes identitaires et sociales (Crits, 1994; Dietler, Herbich, 1994; Gosselain, 2002, p. 11).

La mise en valeur optimale des données liées à ces domaines de recherche à part entière exige la validation des constructions typochronologiques et culturelles. De plus, la qualité des productions céramiques et les méthodes d'analyse sont ici importantes. Pour le sud de la France, les études ne sont le plus souvent menées que sur un matériel très fragmentaire. Dès lors, on ne peut raisonnablement pas accéder de près aux différentes étapes de la chaîne opératoire d'un point de vue technologique (préparation de la pâte, montage, traitement, cuisson...) et à la question des matières premières, comme l'ont proposé C. Colas, F. Giligny, R. Martineau ou encore A. Gelbert dans plusieurs travaux sur les macrotraces observées sur des formes entières archéologiques ou ethnographiques (Colas, 2000; Giligny, 1993 et 1997; Martineau, Pétrequin, 2000; Martineau, 2000 et 2006; Gelbert, 2003 et 2005).

\section{MISE EN PRATIQUE DES DIFFÉRENTS PARTIS PRIS}

Le bilan critique des cent dernières années de recherches livré ici a permis de démêler la construction des théories en cours dans le sud-est de la France. Cette lecture historiographique constitue surtout le point de départ à cette nouvelle analyse que nous avons menée dans le cadre de notre thèse de doctorat (Cauliez, 2009). En optant pour une approche polythétique, ce travail se veut résolument fondé sur une méthodologie diamétralement opposée: c'est-à-dire sur une description exhaustive de grandes séries céramiques, entendues dans leur valeur d'assemblage et provenant de contextes fiables avec l'élaboration d'un protocole descriptif fondé sur une nouvelle terminologie et une typologie de tous les produits céramiques du Néolithique final méridional. Vingt-six séries distinctes sélectionnées pour la validité de leur assemblage ont ainsi été décrites et analysées entièrement à la façon d'une monographie de site. Elles sont issues de dix-huit gisements distribués sur l'ensemble de la Provence, mais aussi hors du cadre régional, depuis le Languedoc, jusque dans le Dauphiné, en passant par le bassin du Rhône moyen. Pour privilégier une lecture dans la continuité et capter les changements et les évolutions, elles s'intègrent également dans une grande partie de la séquence chronologique du Néolithique final, depuis la fin du Néolithique moyen au passage à l'âge du Bronze. Une fois ce stade dépassé, nous fournissons une approche statistique, la seule à même de contrôler et d'exploiter une masse de données qualitatives et quantitatives aussi importante (analyses multivariées, diagonalisations des matrices de présence-absence, indices de Jaccard). Cette dernière nous permet de reconnaître de nouveaux ensembles typostylistiques faisant l'économie de l'attribution culturelle admise pour les séries. À l'aide d'un catalogue raisonné des sites du Néolithique final dans le secteur étudié et grâce à de nouvelles données chronométriques (datations ${ }^{14} \mathrm{C}$ ) obtenues à l'occasion de ce mémoire, ces ensembles typostylistiques sont ensuite abordés sous l'angle chronologique et géographique de manière à définir leur distribution dans le temps et dans l'espace au sein d'une nouvelle périodisation que nous avons produite pour la fin du Néolithique du sud-est de la France. Suivant les modèles polythétiques de D.-L. Clarke, ces ensembles sont articulés les uns avec les autres, pour révéler des liens d'affinité ou d'antagonisme et identifier les mécanismes de formation et d'évolution dans lesquels ils s'inscrivent. Bien loin des premiers canevas, le schéma chronoculturel alternatif défendu alors montre qu'il vaut finalement mieux envisager un Néolithique final aux multiples facettes dans lequel diverses sphères d'influences interagissent. 


\section{BIBLIOGRAPHIE}

\begin{tabular}{ll}
\multicolumn{1}{c}{ ABRÉVIATIONS } \\
ADALR & Association pour le développement de l'archéologie en Languedoc-Roussillon. \\
ADRAHP & Association pour le développement de la recherche archéologique et historique en Périgord. \\
APDCA & Association pour la promotion et la diffusion des connaissances archéologiques. \\
APRAIF & Association pour la promotion de la recherche archéologique en Île-de-France. \\
ARALO & Association pour la recherche archéologique en Languedoc oriental. \\
BAR & British Archaeological Reports. \\
BSPF & Bulletin de la Société préhistorique française. \\
CAP & Centre d'archéologie préhistorique. \\
CRA & Centre de recherches archéologiques. \\
CRHIPA & Centre de recherche en histoire et histoire de l'art : Italie, pays alpins. \\
CTHS & Comité des travaux historiques et scientifiques. \\
ERAUL & Études et recherches archéologiques de l'université de Liège. \\
MSH & Maison des sciences de l'homme. \\
SLP & Société languedocienne de Préhistoire. \\
SPF & Société préhistorique française. \\
SPR & Société préhistorique rhodanienne. \\
SRA & Service régional d'archéologie. \\
UISPP & Union internationale des sciences préhistoriques et protohistoriques.
\end{tabular}

AMBert P.

1990-1991: Le Chalcolithique en Languedoc et ses relations extrarégionales, hommage au $D^{r}$ Jean Arnal, Actes du colloque international, SaintMathieu-de-Tréviers, Hérault, 20-22 sept. 1990, Lattes, Fédération archéologique de l'Hérault (coll. Archéologie en Languedoc, 15), $352 \mathrm{p}$.

1998: "L'évolution de la métallurgie dans le midi de la France», in FRÈRESAUtot M.-C. (DIR.), Paléométallurgie des cuivres, Actes du colloque de Bourg-en-Bresse et Beaune, 17-18 oct. 1997, Montagnac, éd. Monique Mergoil (coll. Monographies Instrumentum, 5), p. 1-15.

\section{AMBERT P., BOUQUET L., GUENDON J.-L., MISCHKA D.}

2005: «La Capitelle du Broum (district minier de Cabrières-Péret, Hérault) : établissement industriel de l'aurore de la métallurgie française (3100-2400 BC) », in Ambert P., VAQUer J. (DIR.), La Première métallurgie en France et dans les pays limitrophes, Actes du colloque international de Carcassonne, 28-30 sept. 2002, Paris, SPF (coll. Mémoires de la SPF, 37), p. 83-96.

Arnal G.-B., DURRenmath G., Gril G.

1991: «L'analyse d'image, méthode de convergence archéométrique: technologie et datation relative", Revue d'archéométrie, 15, p. 103-115.
ARnal J.

1953: «La structure du Néolithique français d'après les récentes stratigraphies", Zephyrus, 4, p. 311-344.

1956: «La grotte de la Madeleine», Zephyrus, 7, p. 33-79.

1963: Les Dolmens du département de l'Hérault, Paris, PUF (coll. Préhistoire, 15), 233 p.

ARNAl J., Bailloud G., RiQuet R.

1960: Les Styles céramiques du Néolithique français: étude didactique, Paris, PUF (coll. Préhistoire, 14), 212 p.

ARNAL J., BURNEZ C.

1958: «Die Struktur des französischen Neolithikums auf Grund neuester stratigraphischer Beobachtungen", Bericht der römisch-germanischen Kommission des deutschen archaeologischen Instituts 19561957, 37-38, p. 1-90.

\section{ARnAl J., Burnez C., Roussot-}

LAROQUE J.

1967: «Sauvetage de la station fontbuxienne du Gravas, Saint-Mathieu-de-Tréviers (Hérault) », BSPF, 64, p. 527-586.

\section{arnal J., Prades H.}

1958: «El Neolítico y Calcolítico franceses», Ampurias, 21, p. 69-164.

\section{AUdiberT J.}

1958: «La période chalcolithique dans le Languedoc oriental», Gallia Préhistoire, 1, p. 39-65.

1962: La Civilisation chalcolithique du Languedoc oriental, Bordighera/Montpellier, Institut international d'études ligures (coll. Monographies préhistoriques et archéologiques, 4), $211 \mathrm{p}$.

BAILlOUd G.

1961: «Les civilisations énéolithiques de la France ", in DE LAET S. J., L'Europe à la fin de l'âge de Pierre, Actes du symposium consacré aux problèmes du Néolithique européen, Prague, Académie tchécoslovaque des sciences, p. 493-508.

1966: «La civilisation du Rhône et le Bronze ancien du midi de la France", Revue archéologique de l'Est, 17, 1-2, p. 131-164.

1970: «Du Néolithique récent au début de l'âge du Bronze ", in GUILAINE J. (DIR.), Les Civilisations néolithiques du midi de la France, Actes du colloque de Narbonne, 15-17 févr. 1970, Carcassonne, Laboratoire de préhistoire et de palethnologie (coll. Atacina, 5), p. 89-93.

1985: «Le Néolithique et le Chalcolithique en France ", in LICHARDUS J., LICHARDUSITTEN M. (DIR.), La Protohistoire de l'Europe: le Néolithique et le Chalcolithique, Paris, PUF (coll. Nouvelle Clio, 1), p. 516568. 


\section{BAILlOUd G., MIEG DE BOOFZHEIM P}

1955: Les Civilisations néolithiques de la France dans leur contexte européen, Paris, Picard, 244 p.

\section{BAILLY M.}

2002: La Flèche et l'éclat: production et consommation des outillages lithiques taillés de la fin du Néolithique au début de l'âge du Bronze entre Saône et Rhône (2600-2000 av. J.-C.), Thèse de Doctorat, Besançon, Université de Franche-Comté, 354 p.

\section{BAIlly M., SAlanova L.}

1999: «Les dates radiocarbone du Campaniforme en Europe occidentale: analyse critique des principales séries de dates", in EVIN J., OBERLIN C., DAUGAS J.-P., SAlles J.-F. (DIR.), ${ }^{14} C$ et archéologie, Actes du $3^{e}$ congrès international, Lyon, 6-10 avril 1998, Paris-Rennes, SPF (coll. Mémoires de la SPF, 26), p. 219-224.

\section{BARGE $\mathbf{H}$.}

1982: Les Parures du Néolithique ancien au début de l'âge des Métaux en Languedoc, Paris, CNRS Éditions, 398 p.

\section{Barge H., Tudiri J., Chauvet A., BARBANSON L.}

2005: Saint-Véran, la montagne, le cuivre et l'homme -I- Mine et métallurgie préhistoriques dans les Hautes-Alpes, Theix, Actilia Multimédia, 83 p.

\section{BARGE-MAHIEU $\mathbf{H}$.}

1995: «Les premiers objets métalliques du Chalcolithique provençal ", in CHENORKIAN R. (DIR.), L'Homme méditerranéen, mélanges offerts à Gabriel Camps, Aix-en-Provence, Publications de l'université de Provence, p. 359-373.

\section{BARGE-MAHIEU H., BORDREUIL M.}

1990-1991: «Révision des pendeloques à ailettes", in AMBERT P. (DIR.), Le Chalcolithique en Languedoc et ses relations extrarégionales, hommage au $D^{r}$ Jean Arnal, Actes du colloque international, SaintMathieu-de-Tréviers, Hérault, 20-22 sept. 1990, Lattes, Fédération archéologique de l'Hérault (coll. Archéologie en Languedoc, 15), p. 183-204.

\section{BARTH F.}

1969: Ethnic Groups and Boundaries: The Social Organization of Culture Difference, Boston, Little, Brown and Company, 153 p.

\section{BEECHING A.}

1980: Introduction à l'étude des stades néolithique et chalcolithique dans le bassin du Rhône moyen: quatre fouilles récentes dans leur contexte régional, Thèse de Doctorat, Université Lyon-II, 3 vol.

1984: «Le couloir rhodanien et ses bordures ", in Influences méridionales dans l'est et le centre-est de la France au Néolithique: le rôle du Massif central, Actes du $8^{e}$ colloque interrégional sur le Néolithique, Le Puy-enVelay, 3-4 oct. 1981, Clermont-Ferrand, Direction régionale des Antiquités préhistoriques d'Auvergne (coll. Cahier du Centre de recherches et d'études préhistoriques de l'Auvergne, 1), p. 259-267.

1986: «Le Néolithique rhodanien, acquis récents et perspectives de la recherche", in Demoule J.-P., GuIlaine J. (DIR.), Le Néolithique de la France, hommage à Gérard Bailloud, Paris, Picard, p. 256-276.

2002: «La fin du Chasséen et le Néolithique final dans le bassin du Rhône moyen", in FERRARI A., VISENTINI P. (DIR.), Il Declino del mondo neolitico: ricerche in Italia centro-settentrionale fra aspetti peninsulari, occidentali e nord-alpini, Atti del convegno, Pordenone, 5-7 aprile 2001, Pordenone, Museo delle scienze (Quaderni del Museo archeologico del Friuli occidentale, 4), p. 67-83.

BEECHING A. avec la collab. de Cordier F., DAUMAS J.-C., LAUDET R., LINOSSIER M., THIERCELIN F.

1995: «Nouveau regard sur le Néolithique ancien et moyen du Bassin rhodanien», in VORUZ J.-L. (DIR.), Chronologies néolithiques: de 6000 à 2000 avant notre ère dans le Bassin rhodanien, Actes du colloque d'Ambérieu-en-Bugey, $19-20$ sept. 1992, $X I^{e}$ rencontre sur le Néolithique de la région Rhône-Alpes, Ambérieu-en-Bugey, SPR (coll. Documents du département d'Anthropologie et d'Écologie de l'université de Genève, 20), p. 93-111.

\section{BERNABÒ BREA L.}

1956: Gli Scavi nella caverna delle Arene Candide, Finale Ligure -I- Gli Stratti con ceramiche-II- Campagne di scavo 1948-1950, Bordighera, Istituto internazionale di studi liguri (coll. Monografie preistoriche ed archeologiche, 1), 296 p.

\section{BESSE M.}

1996: Le Campaniforme en France: analyse de la céramique d'accompagnement, Oxford, Tempus Reparatum (coll. BAR, International Series, 635), $56 \mathrm{p}$.
2004: «Des Campaniformes européens au Campaniforme méditerranéen ", BSPF, 101, 2, p. 215-222.

BILL J.

1973: Die Glockenbecherkultur und die frühe Bronzezeit im französischen Rhonebecken und ihre Beziehungen zur Südwestschweiz, Verlag Schweizerische Gesellschaft für Ur-und Frühgeschichte, Bâle, 111 p.

\section{BINDER D.}

2003: «Considérations préliminaires sur le Néolithique final de l'abri de Pendimoun (Castellar, AlpesMaritimes) ", in GASCÓ J., GUTHERZ X., LABRIFFE P.-A. DE (DIR.), Temps et espaces culturels du VIe au II millénaire en France du Sud, Actes des $I V^{e}$ rencontres méridionales de Préhistoire récente, Nîmes, 28-29 oct. 2000, Lattes, ADALR (coll. Monographies d'archéologie méditerranéenne, 15), p. 293 298 .

BINDER D. avec la collab. de BARBIER M., GUILBERT R.

1998: Recensement des disponibilités en matières premières lithiques de la région Provence-AlpesCôte d'Azur, Rapport de synthèse sur les prospections thématiques en Provence rhodanienne 1995-1997, Aix-en-Provence, UMR 6636 CNRS, Université de Provence, Ministère de la Culture et de la Communication, $16 \mathrm{p}$.

Binder D., Courtin J. (DIR.)

1994: Terre cuite et société: la céramique, document technique, économique, culturel, Actes des $X I V^{e}$ rencontres internationales d'archéologie et d'histoire d'Antibes, 21-23 oct. 1993, Juan-les-Pins, APDCA, 497 p.

BINDER D., ÉCHALLIER J.-C.

1991 : «La céramique», in BINDER D. (DIR.), Une économie de chasse au Néolithique ancien : la grotte Lombard à Saint-Vallier-de-Thiey (Alpes-Maritimes), Paris, CNRS Éditions (coll. CRA-Monographies, 5), p. 71-113.

BINDER D., LEPÈRE C., MAGgi R.

2008: «Épipaléolithique et Néolithique dans l'arc liguro-provençal: bilan et perspectives de recherche», Bulletin du musée d'Anthropologie préhistorique de Monaco, supplément 1, p. 49-62.

BLAISE E.

2005: «L'élevage au Néolithique final dans le sud-est de la France: éléments de réflexion sur la gestion des troupeaux", Anthropozoologica, 40, 1, p. 191-216. 
2007: «La gestion des troupeaux au Néolithique final en Provence: l'exemple $\mathrm{du}$ Couronnien", in FOUÉRÉ P., Chevillot C., Courtaud P., Ferullo O., Leroyer C. (DIR.), Paysages et peuplement: aspects culturels et chronologiques en France méridionale, Actes des $V I^{e}$ rencontres méridionales de Préhistoire récente, Périgueux, 14-16 oct. 2004, Périgueux, ADRAHP (coll. Suppl. à Préhistoire du Sud-Ouest, 11), p. 433-442.

\section{BOCQUET A.}

1997: «Archéologie et peuplement des Alpes françaises du Nord au Néolithique et aux âges des Métaux ", L'Anthropologie, 101, 2, p. 291-393.

\section{BOISSINOT P.}

1998: «Que faire de l'identité avec les seules méthodes de l'archéologie ?", in D'Anna A., BInder D. (DIR.), Production et identité culturelle: actualité de la recherche, Actes des II ${ }^{e}$ rencontres méridionales de Préhistoire récente, Arles, 8-9 nov. 1996, Antibes, APDCA, p. 17-25.

\section{BORDREUIL M.}

1995: «Recherches sur les chronologies du Néolithique final dans la région d'Alès: le Fontbouisse baroque, une mode à part entière ?", in VORUZ J.-L. (DIR.), Chronologies néolithiques: de 6000 à 2000 avant notre ère dans le Bassin rhodanien, Actes du colloque d'Ambérieu-en-Bugey, 19-20 sept. 1992, XI rencontre sur le Néolithique de la région Rhône-Alpes, Ambérieu-en-Bugey, éd. SPR (coll. Documents du département d'anthropologie et d'écologie de l'université de Genève, 20), p. 307-311.

1998: «À propos des faciès du Néolithique final en Languedoc oriental: l'hypothèse du groupe des Bruyères", in D'ANNA A., BINDER D. (DIR.), Production et identite culturelle: actualité de la recherche, Actes des $I I^{e}$ rencontres méridionales de Préhistoire récente, Arles (Bouches-du-Rhône), 8-9 nov. 1996, Antibes, APDCA, p. 329-341.

\section{BOURDIEU P.}

1979: La Distinction: critique sociale du jugement, Paris, éd. de Minuit (coll. Le Sens commun), $680 \mathrm{p}$.

\section{BRETAGNe P., D'ANNA A}

1988: «Bonnieux, les Fabbri», Notes d'information et de liaison de la Direction des Antiquités de Provence-Alpes-Côte d'Azur, 5, p. 163-166.
BRetagne P., Helmer D., MEFFre J.

1986: Gigondas, les Vignes de St-André, 1986: fouille de sauvetage urgent, Rapport dactylographié, $15 \mathrm{f}$.

\section{BUisson-GATIL J., GUILChER A.,}

Pagni M., Olive M., HUSSY C.

2004: Vaucluse préhistorique: le territoire, les hommes, les cultures et les sites, Le Pontet, éd. Barthélémy (coll. Archéologie), $320 \mathrm{p}$

\section{CAMPS G.}

1989: «La Provence préhistorique», in FÉVrier P.-A. (DIR.), La Provence des origines à l'an mil: histoire et archéologie, Rennes, éd. Ouest-France université (coll. Histoire de la Province), p. 55-166.

Camps-Fabrer H., Carry A., Sauzade G.

1983: «L'industrie osseuse du site de Claparouse, Lagnes (Vaucluse)", in Congrès préhistorique de France, compte rendu de la XXI session, Montauban-Cahors (Quercy), 3-9 sept. 1979, t. 1, Paris, SPF, p. $45-56$.

CAMPS-FABRER H., D'ANNA A.

1989: «Enceintes et structures d'habitat du Néolithique final, Miouvin 3 (Istres, Bouches-du-Rhône) ", in D'ANNA A., GUTHERZ X. (DIR.), Enceintes, habitats ceinturés, sites perchés du Néolithique au Bronze ancien dans le sud de la France et les régions voisines, Actes de la table ronde de Lattes, Aix-en-Provence, 15-18 avril 1987, Montpellier, SLP (coll. Mémoires de la SLP, 2), p. 196-208.

\section{CARboni G.}

2002: «Territorio aperto o di frontiera? Nuove prospettive di ricerca per lo studio della distribuzione spaziale delle "facies" del Gaudo e di Rinaldone nel Lazio centro-meridionale», Origini: preistoria e protoistoria delle civiltà antiche, 24, p. 235-301.

\section{Carozza L.}

2000: «Économie et territoire aux débuts de la métallurgie dans la moyenne vallée de l'Hérault: émergence d'une problématique ", in LEDUC M., VALDEYRON N., VAQUER J. (DIR.), Sociétés et espaces: actualité de la recherche, Actes des III rencontres méridionales de Préhistoire récente, Toulouse, 6-7 nov. 1998, Toulouse, Archives d'écologie préhistorique, p. 157-175.

2002: La Fin du Néolithique et les débuts de la métallurgie en Languedoc central: innovation technique et mutations sociales des villages ceinturés du Puech-Haut (Paulhan, Hérault), 3500 et 2200 av. J.-C., Document final de synthèse, SRA Languedoc-Roussillon, 2 vol.

\section{CAUliez J.}

2001a: «Redéfinition du groupe culturel Rhône-Ouvèze et la question du Fontbouisse en Provence: implications pour l'étude du Couronnien. Présentation des résultats de l'étude de la céramique du site de la Fare à Forcalquier (Alpesde-Haute-Provence) ", in LEMERCIER O. (DIR.), Le Couronnien en Basse-Provence occidentale: état des connaissances et nouvelles perspectives de recherches, Rapport d'activité 2001, Aix-en-Provence, ESEP/ SRA Provence-Alpes-Côte d'Azur, p. 175184.

2001b: «Incidence du groupe couronnien sur la genèse du Rhône-Ouvèze: quelques pistes d'une démarche comparative ", in LEMERCIER O. (DIR.), Le Couronnien en Basse-Provence occidentale: état des connaissances et nouvelles perspectives de recherches, Rapport d'activité 2001, Aix-en-Provence, ESEP/SRA Provence-Alpes-Côte d'Azur, p. 172-186.

2002: La Céramique du Néolithique final dans le sud-est de la France: le groupe du RhôneOuvèze entre faciès fontbuxien et groupe à part entière, Mémoire de DEA, Université AixMarseille-I, Aix-en-Provence, 2 vol., 97 p.

2007: «Les corpus céramiques du III ${ }^{\mathrm{e}}$ millénaire av. J.-C. dans le sud-est de la France: identité du groupe Rhône-Ouvèze", $B S P F, 104,1$, p. 125-145.

2009: Espaces culturels et espaces stylistiques au Néolithique final dans le sud-est de la France: dynamiques de formation et d'évolution des productions céramiques, Thèse de Doctorat, Université Aix-Marseille-I, Aixen-Provence, 4 vol., 1269 p.

À paraître a: «Le Couronnien et son implication dans la mise en place du groupe Rhône-Ouvèze: un état de la question", in LEMERCIER O. (DIR.), Le Couronnien en Basse-Provence occidentale: état des connaissances et nouvelles perspectives de recherches, Rapport d'activité 2005, Aix-en-Provence, ESEP/SRA Provence-Alpes-Côte d'Azur.

À paraître b: «Le Collet-Redon (la Couronne, Martigues, Bouches-du-Rhône) et le Couronnien: la céramique de l'habitation $\mathrm{n}^{\circ} 1$ ", in LEMERCIER O. (DIR.), Le Couronnien en Basse-Provence occidentale: état des connaissances et nouvelles perspectives de recherches, Rapport d'activité 2005, Aixen-Provence, ESEP/SRA Provence-AlpesCôte d'Azur. 
Cauliez J., Blaise E., Desse-Berset N., Desse J., Cade C., DURrenmath G., Gilabert C., Martin S., Vella C.

2007: «Paysage et implantations du Néolithique final à l'âge du Bronze ancien au Collet-Redon (Martigues, Bouches-duRhône)", in FouÉRÉ P., CHEVIllot C., Courtaud P., Ferullo O., LeRoyer C. (DIR.), Paysages et peuplement: aspects culturels et chronologiques en France méridionale, Actes des $V I^{e}$ rencontres méridionales de Préhistoire récente, Périgueux, 14-16 oct. 2004, Périgueux, ADRAHP (coll. Suppl. à Préhistoire du Sud-Ouest, 11), p. 125-140.

\section{Cazalis de Fondouce P.}

1867: Derniers temps de l'âge de la pierre polie dans l'Aveyron: la grotte sépulcrale de SaintJean-d'Alcas et les dolmens de Pilande et des Costes, Savy, Montpellier, 1867, 90 p.

1900: Géographie générale du département de l'Hérault, 3, -1- L'Hérault aux temps préhistoriques, $195 \mathrm{p}$.

\section{CEUNINCK G. DE}

1994: «Forme, fonction, ethnie: approche ethnoarchéologique des céramiques du delta inférieur du Niger (Mali)», in BINDer D., COURTIN J. (DIR.), Terre cuite et société: la céramique, document technique, économique, culturel, Actes des $X I V^{E}$ rencontres internationales d'archéologie et d'histoire d'Antibes, 21-23 oct. 1993, Juanles-Pins, APDCA, p. 161-177.

\section{CHANTRE E.}

1875-1876: Études paléoethnologiques dans le bassin du Rhône: âge du Bronze, recherches sur les origines de la métallurgie en France, Paris, Librairie polytechnique J. Baudry, 321 p.

\section{CHOI S.-Y.}

1999: Outillages en matière dure animale du Néolithique ancien au Chalcolithique dans le midi de la France: étude technique et morphologique, Thèse de Doctorat, Université Aix-Marseille-I, Aix-en-Provence, 851 p.

\section{Clarke D.-L.}

1978: Analytical Archaeology, Londres, Methuen \& Co Ltd, 526 p.

\section{GLERMONT N.}

1999: «L'archéologue, la culture matérielle et les problèmes de l'ethnicité", Recherches amérindiennes au Québec, 29, 1 p. 71-73.

Colas C.

2000: Savoir-faire technique et reconstitutions des chaînes opératoires des potiers au Néolithique moyen II dans la moitié nord de la
France: étude techno-typologique, Thèse de Doctorat, Université Paris-I, 3 vol., 572 p.

CONKEY M. W.

1990: The Uses of Style in Archaeology, Cambridge, Cambridge University Press, $136 \mathrm{p}$.

\section{Conti A. M., Persiani C., Petitti P.}

1997: «I riti della morte nella necropoli eneolitica della selvicciola (Ischia di Castro-Viterbo) ", Origini: preistoria e protoistoria delle civiltà antiche, 21, p. 169-186.

\section{CONVERTINI F.}

1996: Production et signification de la céramique campaniforme à la fin du III ${ }^{e}$ millénaire av. J.-C. dans le sud et le centre-ouest de la France et en Suisse occidentale, Oxford, Tempus Reparatum (coll. BAR International Series, 656), $372 \mathrm{p}$.

1998: «Identification de marqueurs culturels dans la céramique du Néolithique du sud-est de la France: apports pour une meilleure compréhension du phénomène campaniforme", in D'ANNA A., BINDER D. (DIR.), Production et identité culturelle: actualité de la recherche, Actes des $I I^{e}$ rencontres méridionales de Préhistoire récente, Arles, 8-9 nov. 1996, Antibes, APDCA, p. 203-215

\section{Convertini F., FUrestier R., Astruc L.,} FOREST V., JALLOT L.

2004: «Le Mas de Vignole IV à Nîmes (Gard) : résultats préliminaires des fouilles d'un fossé à occupation campaniforme", in DARTEVelle H. (DIR.), Auvergne et Midi, Actes des Ve rencontres méridionales de Préhistoire récente, Clermont-Ferrand, 8-9 nov. 2002, Cressensac, Préhistoire du Sud-Ouest (coll. Suppl. à Préhistoire du Sud-Ouest, 9), p. 493-507.

\section{Cornaggia Castiglioni O.}

1971: La Cultura di Remedello: problematica ed ergologia di una facies dell'Eneolitico Padano, Milan, Succ. Fusi (coll. Memorie della Società italiana di Scienze naturali e del Museo civico di Storia naturale di Milano, 20), 79 p.

\section{COURTIN J.}

1960: «La station du Pilon du Roy à Allauch (Bouches-du-Rhône)» Gallia Préhistoire, 3, p. 142-149.

1970 : «Le Néolithique récent de la Provence», in GUILAINE J. (DIR.), Les Civilisations néolithiques du midi de la France, Actes du colloque de Narbonne, 15-17 févr. 1970, Carcassonne, Laboratoire de préhistoire et de palethnologie (coll. Atacina 5), p. 121-123.

1974: Le Néolithique de la Provence, Paris, Klincksieck (coll. Mémoires de la SPF, 11), $359 \mathrm{p}$.

1975: «Un habitat fortifié du Bronze ancien en Basse-Provence: le camp de Laure», Bulletin du Muséum d'histoire naturelle de Marseille, 35, p. 218-240.

1976: «Les civilisations néolithiques en Provence", in GuILAINE J. (DIR.), La Préhistoire française -II- Les Civilisations néolithiques et protohistoriques de la France, Paris, CNRS Éditions, p. 255-266.

1977: «L'habitat de plein air du Pilon du Roy (Allauch, Bouches-du-Rhône) et la céramique à chevrons en relief du Néolithique final et du Chalcolithique provençaux ", Bulletin du Muséum d'histoire naturelle de Marseille, 37, p. 129-140.

Courtin J., Sauzade G.

1975: «Un poignard de type Remedello en Provence », BSPF, 72, 6, p. 184-190.

\section{Cremonesi G.}

1998: «L'Italie centrale», in GUILAINE J. (DIR.), Atlas du Néolithique européen -IIEurope occidentale, Liège, Université de Liège (coll. ERAUL, 46), p. 165-231.

\section{CRITS E. DE}

1994: «Style et technique: comparaison interethnique de la poterie subsaharienne", in BINDER D., COURTIN J. (DIR.), Terre cuite et société: la céramique, document technique, économique, culturel, Actes des XIV rencontres internationales d'archéologie et d'histoire d'Antibes, 21-23 oct. 1993, Juanles-Pins, APDCA, p. 343-350.

\section{D'ANNA A.}

1989: «L'habitat perché Néolithique final de la Citadelle (Vauvenargues, Bouchesdu-Rhône) ", in D'ANNA A., GUTHERZ X. (DIR.), Enceintes, habitats ceinturés, sites perchés du Néolithique au Bronze ancien dans le sud de la France et les régions voisines, Actes de la table ronde de Lattes, Aix-en-Provence, 15-18 avril 1987, Montpellier, SLP (coll. Mémoires de la SLP, 2), p. 209-224.

1991: "L'habitat de plein air Néolithique final et Chalcolithique en Provence", Bulletin de l'école antique de Nîmes, 22, p. 31-42.

1993: «L'habitat de plein air en Provence: recherches récentes ", in BLANCHET J.-C., Bulard A., Constantin C., (DIR.), Le Néolithique au quotidien, Actes du XVI $\mathrm{col}$ loque interrégional sur le Néolithique, Paris, 5-6 nov. 1989, Paris, éd. de la MSH (coll. 
Documents d'archéologie française, 39), p. $72-84$.

1995a: «La fin du Néolithique dans le sudest de la France», in CHENORKIAN R. (DIR.), L'Homme méditerranéen, mélanges offerts à Gabriel Camps, Aix-en-Provence, Publications de l'université de Provence, p. 299-333.

1995b: «Le Néolithique final en Provence», in VORUZ J.-L. (DIR.), Chronologies néolithiques: de 6000 à 2000 avant notre ère dans le Bassin rhodanien, Actes du colloque d'Ambérieu-en-Bugey, 19-20 sept. 1992, XI rencontre sur le Néolithique de la région Rhône-Alpes, Ambérieu-en-Bugey, SPR (coll. Documents du département d'Anthropologie et d'Écologie de l'université de Genève, 20), p. 265-286.

1999: «Le Néolithique final en Provence», in VAQUER J. (DIR.), Le Néolithique du Nord-Ouest méditerranéen, Actes du XXIV congrès préhistorique de France, Carcassonne, 26-30 sept. 1994, Paris, SPF, p. 147-159.

\section{D'Anna A., Courtin J., Coutel R., MÜller A.}

1989: «Habitats perchés et enceintes du Néolithique final et chalcolithique dans le Luberon central (Vaucluse)", in D'ANNA A., GUTHERZ X. (DIR.), Enceintes, habitats ceinturés, sites perchés du Néolithique au Bronze ancien dans le sud de la France et les régions voisines, Actes de la table ronde de Lattes, Aix-en-Provence, 15-18 avril 1987, Montpellier, SLP (coll. Mémoires de la SLP, 2), p. 165-193.

\section{D'AnNa A., Renault S., GUENDON J.-L.,} MASSE J.-P., PINET L., WALTER P.

2004: Stèles anthropomorphes néolithiques de Provence, Catalogue du musée Calvet d'Avignon, Avignon, Établissement public Calvet, 96 p.

\section{Delaunay G.}

2001 : «Le mobilier des Lauzières (Lourmarin, Vaucluse) : problématiques chronoculturelles et premières observations sur la zone VI", in LEMERCIER O. (DIR.), Le Couronnien en Basse-Provence occidentale: état des connaissances et nouvelles perspectives de recherches, Rapport d'activité 2001, Aixen-Provence, ESEP/SRA Provence-AlpesCôte d'Azur, p. 77-85.

\section{DELESTRE X., BUISSON-CATIL J.}

2006: Les Grandes découvertes en Préhistoire dans la région Provence-Alpes-Côte d'Azur, Aixen-Provence, Édisud (coll. Archéologie méditerranéenne), $152 \mathrm{p}$.

\section{De Marinis R. C., Pedrotti A.}

1997: "L'età del rame nel versante italiano delle Alpi centro-occidentali -3- La cultura di Remedello: nuove proposte di cronologia relativa e assoluta», in La Valle d'Aosta nel quadro della Preistoria e Protostoria dell'arco alpino centro-occidentale, Atti della XXXI riunione scientifica, Courmayeur, 2-5 giugno 1994, Florence, Istituto italiano di preistoria e protostoria, p. 247-300.

\section{DEMOULE J.-P.}

1994: «La céramique comme marqueur social: variabilité spatiale et chronologique », in BINDER D., COURTIN J. (DIR.), Terre cuite et société: la céramique, document technique, économique, culturel, Actes des $X I V^{E}$ rencontres internationales d'archéologie et d'histoire d'Antibes, 21-23 oct. 1993, Juanles-Pins, APDCA, p. 473-492.

\section{DIETLER M., HERBICH I.}

1994: "Ceramics and Ethnic Identity: Ethnoarcheological Observations on the Distribution of Pottery Styles and the Relationship between the Social Contexts of Production and Consumption", in BINDER D., COURTIN J. (DIR.), Terre cuite et société: la céramique, document technique, économique, culturel, Actes des XIV rencontres internationales d'archéologie et d'histoire d'Antibes, Antibes, 21-23 oct. 1993, Juan-les-Pins, APDCA, p. 459-472.

Di Pierro S., Michel R., Martineau R. 2005: «Matériaux et types céramiques à Saint-Blaise, station néolithique suisse (2770-2626 av. J.-C.) : poterie exogène et production locale", in LIVINGSTONE SMith A., Bosquet D., Martineau R. (DIR.), Pottery Manufacturing Processes: Reconstitution and Interpretation, Acts of the XIVth UISPP Congress, University of Liège, Belgium, 2-8 sept. 2001, Colloque/ Symposium 2.1, Cambridge, Archaeopress (coll. BAR International Series, 1349), p. 157-177.

\section{DUfRAigne J.-J., Guillet E., JALlet F.}

2003: «Les Pins (Aubais, Gard) : un établissement du début du Bronze ancien", in Pirineus $i$ veïns al $3^{r}$ mil.leni aC. De la fi del Neolític a l'edat del Bronze entre l'Ebre $i$ la Garona: homenatge al prof. Domènec Campillo, XII Col.loqui internacional d'arqueologia de Puigcerdà, nov. 2000, Puigcerdà, Insitut d'Estudis Ceretans.

\section{DUPUY F.}

2004: Anthropologie économique, Paris, Armand Colin (coll. Cursus, Sociologie générale), $216 \mathrm{p}$.

\section{DURAND J.}

1999: «Les pointes de Sigottier: fait technoculturel et marqueur géographique», in BEECHING A. (DIR.), Circulations et identités culturelles alpines à la fin de la Préhistoire: matériaux pour une étude, Valence, CAP (coll. Travaux du CAP, 2), p. 231-257.

2002: «Occupations néolithiques du site de Roynac-Le Serre (Drôme)», in GUTHERZ X. (DIR.), Archéologie du TGV Méditerranée, fiches de synthèse -1- La Préhistoire, Lattes, ARALO (coll. Monographies d'archéologie méditerranéenne, 8), fiche 6, p. 67-84.

\section{DURRENMATH G.}

1998: «Contraintes, typologies, cultures: abord quantitatif du dégraissant de céramiques du Néolithique final de Provence», in D'ANNA A., BINDER D. (DIR.), Production et identité culturelle: actualité de la recherche, Actes des II rencontres méridionales de Préhistoire récente, Arles, 8-9 nov. 1996, Antibes, APDCA, p. $187-202$.

2001: Étude quantitative du dégraissant de céramiques préhistoriques de Provence par analyse d'images: essai de différenciation typologique, culturelle et chronologique, Thèse de Doctorat, Université Aix-Marseille-I, Aix-en-Provence, 318 p.

2005: "Technologie céramique et savoirs métallurgiques: le dégraissant des productions provençales de la seconde moitié du III ${ }^{\mathrm{e}}$ millénaire avant notre ère", in AMBert P., VAQUER J. (DIR.), La Première métallurgie en France et dans les pays limitrophes, Actes du colloque international, Carcassonne, 28-30 sept. 2002, Paris, SPF (coll. Mémoires de la SPF, 37), p. 151-157.

Durrenmath G., Cauliez J., Blaise E., CAde C., Desse J., DesSe-Berset N.

2007: «Le Collet-Redon (Martigues, Bouchesdu-Rhône) : passé, présent et futur des recherches sur l'économie d'un site néolithique final», in EVIN J. (DIR.), Un siècle de construction du discours scientifique en Préhistoire: des idées d'hier aux conceptions d'aujourd'hui, Actes du XXVI congrès préhistorique de France, congrès du centenaire de la SPF, Avignon, 21-25 sept. 2004, Paris, SPF, vol. 3, p. 387-399. 


\section{DUrRenmath G., Cauliez J.,} GILABERT C.

À paraître: "L'habitation $\mathrm{n}^{\circ} 1$ du ColletRedon à la Couronne (Martigues, Bouches-du-Rhône): relecture critique des sources d'un site éponyme», L'Anthropologie.

DURRENMATH G., LUZI C., FURESTIER R., Gilabert C., PELlissier M., LAZARD N., Provenzano N.

2003: "Les occupations du Collet-Redon (Martigues, Bouches-du-Rhône): l'enceinte de l'âge du Bronze ", in GASCÓ J., GUTHERZ X., LABRIFFE P.-A. DE (DIR.), Temps et espaces culturels du VI $I^{e}$ au II $I^{e}$ millénaire en France du Sud, Actes des IV rencontres méridionales de Préhistoire récente, Nîmes, 28-29 oct. 2000, Lattes, ADALR (coll. Monographies d'archéologie méditerranéenne, 15), p. 263-269.

\section{ÉCHALLIER J.-C., THIERCELIN F.}

1991: «Premières données pétrographiques sur les céramiques chasséennes de la moyenne vallée du Rhône", in BEECHING A., BINDER D., Blanchet J.-C., Constantin C., DUbouloz J., MARTINEZ R., MORDANT D., THÉVENOT J.-P., VAQUER J. (DIR.), Identité du Chasséen, Actes du colloque international de Nemours, 17-19 mai 1989, Nemours, APRAIF (coll. Mémoires du musée de Préhistoire d'Île-de-France, 4), p. 293-297.

\section{ESCALON DE FONTON M.}

1947: «Découverte d'une station en plein air à La Couronne (Bouches-du-Rhône)», Mémoires de l'Institut historique de Provence, 22, p. 33-43.

1954: «Tour d'horizon de la Préhistoire provençale», $B S P F, 51,1-2$, p. 81-96.

1955: «Nouvelles vues sur la chronologie du Néo-Énéolithique ", Provence historique, 5, p. 97-102.

1956: Préhistoire de la Basse-Provence-12- État d'avancement des recherches en 1951, Paris, PUF, $170 \mathrm{p}$.

1958: «La valeur chronologique relative de la stratigraphie du Néolithique», Gallia Préhistoire, 1, p. 79-92.

1963: "Origine et développement des civilisations néolithiques méditerranéennes en Europe occidentale», Cahiers ligures de Préhistoire et d'archéologie, 12, 2, p. 177-180.

1964: «Recherches sur la préhistoire dans le Midi de la France: rapport préliminaire sur la campagne de fouilles de 1963", Cahiers ligures de Préhistoire et d'archéologie, 13, 2, p. 251-266.
1968: Préhistoire de la Basse-Provence occidentale, Martigues, Office du tourisme de la région de Martigues, $71 \mathrm{p}$.

1974: «Informations archéologiques: Circonscription de Provence Côte-d'Azur», Gallia Préhistoire, 17, 2, p. 665-699.

\section{FURESTIER R.}

2002: "L'industrie lithique des sites couronniens: un état de la question", in LEMERCIER O. (DIR.), Le Couronnien en Basse-Provence occidentale: état des connaissances et nouvelles perspectives de recherches, Rapport d'activité 2002, Aix-en-Provence, ESEP/SRA Provence-Alpes-Côte d'Azur, p. 118-143.

2005: Les Industries lithiques campaniformes $d u$ sud-est de la France, Thèse de Doctorat, Aix-en-Provence, Université AixMarseille-I, vol. 1, 328 p.

FUrestier R., CaUliez J., Lazard N., Lemercier O., Pellissier M., Courtin J. 2007: «1974-2004: le site du Fortin-du-Saut (Châteauneuf-les-Martigues, Bouchesdu-Rhône) et le Campaniforme 30 ans après", in EVIN J. (DIR.), Un siècle de construction $d u$ discours scientifique en Préhistoire: des idées d'hier aux conceptions d'aujourd'hui, Actes du XXVI congrès préhistorique de France, congrès du centenaire de la SPF, Avignon, 21-25 sept. 2004, vol. 2, p. $297-310$.

\section{GAGNIÈRE S}

1961: «Informations archéologiques: circonscription d'Aix-en-Provence», Gallia Préhistoire, 4, p. 337-386.

\section{GallaY A.}

1976: «Origine et expansion de la civilisation du Rhône ", in BOCQUET A., LAGRAND C. (DIR.), Néolithique et âges des Métaux dans les Alpes françaises, Actes du IX $X^{e}$ congrès de l'UISPP, Nice, 1976, livret guide de l'excursion A9, Nice, Université de Nice SophiaAntipolis, p. 5-26.

1995: «Pour un modèle de la société néolithique", in STÖCKLI W. E., NIFFELER U., Gross-KLEE E. (DIR.), Néolithique, Bâle, Société suisse de Préhistoire et d'archéologie (coll. La Suisse du Paléolithique à l'aube du Moyen Âge, 2), p. 275-288.

1996: «Le concept de culture du Rhône: repères pour un historique", in MORDANT C., GAIFFE O. (DIR.), Cultures et sociétés du Bronze ancien en Europe, Actes du $117^{\circ}$ congrès national des sociétés savantes, Clermont-Ferrand, 1992, Paris, éd. du CTHS (coll. Actes des congrès nationaux des sociétés historiques et scientifiques), p. 271-286.
Gallay A., Ceuninck G. DE

1998: «Les jarres de mariage décorées du delta intérieur du Niger (Mali) approche ethnoarchéologique d'un "bien de prestige" ", in FRITSCH B., MaUte M., Matuschik I., MÜller J., WOLF C. (DIR.), Tradition und Innovation: Festschrift für Christian Strahm, Rahden, Marie Leidorf (coll. Internationale Archäologie, Studia honoraria, 3), p. 13-30.

GASCó J.

1976: La Communauté paysanne de Fontbouisse, Carcassonne, Centre d'écologie humaine (coll. Archives d'écologie préhistorique, 1), $121 \mathrm{p}$.

1980: «Un habitat de plein air au Néolithique récent: la Mort des Ânes (Villeneuve-lèsMaguelone, Hérault)», in GUILAINE J. (DIR.), Le Groupe de Véraza et la fin des temps néolithiques dans le sud de la France et la Catalogne, Actes du colloque de Narbonne, 3-4 juin 1977, Paris, CNRS Éditions, p. 177-191.

1990-1991: «La chronologie absolue du Néolithique final et du Chalcolithique en Languedoc méditerranéen", in AMBERT P. (DIR.), Le Chalcolithique en Languedoc et ses relations extrarégionales, hommage au $D^{r}$ Jean Arnal, Actes du colloque international, Saint-Mathieu-deTréviers, Hérault, 20-22 sept. 1990, Lattes, Fédération archéologique de l'Hérault (coll. Archéologie en Languedoc, 15), p. 217-255

\section{GELBERT A.}

2003: Traditions céramiques et emprunts techniques dans la vallée du fleuve Sénégal, Paris, éd. de la MSH et éd. Épistèmes, $104 \mathrm{p}$

2005: «Reconnaissance des techniques et des méthodes de façonnage par l'analyse des macrotraces: étude ethnoarchéologique dans la vallée du Sénégal», in LiVIngSTONE SMITH A., BosQUeT D., Martineau R. (DIR.), Pottery Manufacturing Processes: Reconstitution and Interpretation, Acts of the XIVth UISPP Congress, University of Liège, Belgium, 2-8 sept. 2001, Colloque/Symposium 2.1, Cambridge, Archaeopress (coll. BAR International Series, 1349), p. 67-78.

\section{GEORJON C. avec la collab. de FOREST V.,} RAUX A.

1999: «Le site de la Roquette à Tresques (Gard) et le Néolithique final du bassin bas rhodanien ", Gallia Préhistoire, 41, p. 253-297. 


\section{GERNIGON K.}

2004: Productions matérielles et identités culturelles dans le Néolithique d'Europe occidentale: réflexions autour de la céramique chasséenne en Quercy, Thèse de Doctorat, Toulouse, Université Toulouse-le-Mirail, 2 vol., $686 \mathrm{p}$

\section{GillaberT C.}

2002: «Vers une nouvelle définition de l'habitat couronnien ", in LEMERCIER O. (DIR.), Le Couronnien en Basse-Provence occidentale: état des connaissances et nouvelles perspectives de recherches, Rapport d'activité 2002, Aixen-Provence, ESEP/SRA Provence-AlpesCôte d'Azur, p. 85-97.

\section{GILIGNY F.}

1993: La Variabilité des récipients céramiques au Chalcolithique moyen récent jurassien (3400-2400 av. J.-C.): analyse archéologique d'un système d'objets, Thèse de Doctorat, Université Paris-I, 4 vol., 882 p.

1994: «Variabilité et transferts techniques dans le Jura à la fin du IV et au III ${ }^{\mathrm{e}}$ millénaire av. J.-C. », in BINDER D., COURTIN J. (DIR.), Terre cuite et société: la céramique, document technique, économique, culturel, Actes des XIV rencontres internationales d'archéologie et d'histoire d'Antibes, 21-23 oct. 1993, Juan-les-Pins, APDCA, p. 363-380.

1997: "La céramique de Chalain 3», in PÉTREQUIN P. (DIR.), Les Sites littoraux néolithiques de Clairvaux-les-Lacs et de Chalain (Jura) -3- Chalain station 3, 32002900 av. J.-C., Paris, éd. de la MSH (coll. Archéologie et culture matérielle), p. $327-370$

Giligny F., Maréchal D., Pétrequin P., PÉTRequin A.-M., SAINTOT S.

1995: «La séquence Néolithique final des lacs de Clairvaux et de Chalain (Jura): essai sur l'évolution culturelle", in VORUZ J.-L. (DIR.), Chronologies néolithiques: de 6000 à 2000 avant notre ère dans le Bassin rhodanien, Actes du colloque d'Ambérieu-en-Bugey, 19-20 sept. 1992, XI rencontre sur le Néolithique de la région Rhône-Alpes, Ambérieu-en-Bugey, SPR (coll. Documents du département d'Anthropologie et d'Écologie de l'université de Genève, 20), p. 313-346.

\section{GILIGNY F., Michel R.}

1995: «L'évolution des céramiques de 2920 av. J.-C. dans la région des Trois-Lacs (Suisse occidentale)", in VORUZ J.-L. (DIR.), Chronologies néolithiques: de 6000 à 2000 avant notre ère dans le Bassin rhodanien, Actes du colloque d'Ambérieuen-Bugey, 19-20 sept. 1992, XI rencontre sur le Néolithique de la région Rhône-Alpes, Ambérieu-en-Bugey, SPR (coll. Documents du département d'Anthropologie et d'Écologie de l'université de Genève, 20), p. 347-361.

Giligny F., Salanova L., DaVid C., DECHEZLEPRÊTRE T., DURAND S., Grouber P., Peake R., Perrin T., PiERRAT J.-M., THÉron V., TIMSIT D., WELLER O.

1997: «La variabilité des corpus céramiques méridionaux au Néolithique final chalcolithique», BSPF, 94, p. 237-258.

\section{GILLES R.}

1975: «L'habitat du Néolithique final des Bruyères (basse vallée de l'Ardèche)", Études préhistoriques, 12, p. 1-13.

\section{GODELIER M.}

1982: La Production des grands hommes, Paris, Fayard (coll. Espace du politique), $400 \mathrm{p}$ 2008: L'Énigme du don, Paris, Flammarion (coll. Champs essais, 783), $316 \mathrm{p}$.

\section{GORDON CHILDE V.}

1949: L’Aube de la civilisation européenne, Paris, Payot, $384 \mathrm{p}$

\section{Gosselain O. P.}

2002: Poteries du Cameroun méridional: styles techniques et rapports à l'identité, Paris, CNRS Éditions (coll. Monographies du CRA, 26), 256 p.

\section{GUILAINE J.}

1967: La Civilisation du vase campaniforme dans les Pyrénées françaises, Carcassonne, Gabelle, $240 \mathrm{p}$

1970: Les Civilisations néolithiques du midi de la France, Actes du colloque de Narbonne, 15-17 févr. 1970, Carcassonne, Laboratoire de préhistoire et de palethnologie (coll. Atacina, 5), 133 p.

1972: L'Âge du Bronze en Languedoc occidental, Roussillon, Ariège, Paris, Klincksieck (coll. Mémoires de la SPF, 9), $460 \mathrm{p}$

1976a: Premiers bergers et paysans de l'Occident méditerranéen, Paris, Mouton/EHESS (coll. Civilisations et sociétés, 58), 296 p.

1976b: «La civilisation des gobelets campaniformes dans la France méridionale", in GUILAINE J. (DIR.), La Civilisation des vases campaniformes, Actes du IX congrès de l'UISPP, Nice, 1976, colloque XXIV, Nice, Prétirages, p. 197-213.

1980: Le Groupe de Véraza et la fin des temps néolithiques dans le sud de la France et la Catalogne, Actes du colloque de Narbonne. 3-4 juin 1977, Paris, CNRS Éditions, $296 \mathrm{p}$.
1986: «Gérard Bailloud ou trente ans de recherches sur le Néolithique français», in Demoule J.-P., Guilaine J. (DIR.), Le Néolithique de la France, hommage à Gérard Bailloud, Paris, Picard, p. 7-14.

1994: La Mer partagée: la Méditerranée avant l'écriture, 7000-2000 av. J.-C., Paris, Hachette Littératures (coll. Beaux Livres), $455 \mathrm{p}$.

2000: Au temps des dolmens: la France méridionale au temps des mégalithes, Toulouse, éd. Privat (coll. Pages Grand Sud), 159 p.

2003: «Un demi-siècle de recherches françaises en archéologie néolithique (19502000) ", La Revue pour l'histoire du CNRS, 8, [URL: http://histoire-cnrs.revues. org/document552.html].

2004: «Les Campaniformes et la Méditerranée», $B S P F, 101,2$, p. 239-249.

2007: Le Chalcolithique et la construction des inégalités -2- Proche et Moyen-Orient, Amérique, Afrique, Séminaire du Collège de France, Paris, éd. Errance (coll. des Hespérides), $135 \mathrm{p}$.

GUILAINE J., BESSE M., LEMERCIER O., SAlanOVa L., STRAHM G., VAN DER LINDEN M.

2004: «Avant-propos: les Campaniformes aujourd'hui ", BSPF, 101, 2, p. 197-200.

Guilaine J., BLANChet J.-C., L'Helgouach J., Pétrequin P., ROUSSOT-LARROQUE J.

1988: «Le Chalcolithique en France», in L'Età del Rame in Europa, Atti del congresso internazionale, Viareggio, 15-18 oct. 1987, Florence, All'Insegna del Giglio (coll. Rassegna di archeologia, 7), p. 211-263.

\section{Guilaine J., Claustre F., Lemercier O.,} SABATIER $P$.

2001: «Campaniformes et environnement culturel en France méditerranéenne», in NICOLIS F. (DIR.), Bell Beakers Today: Pottery, People, Culture, Symbols in Prehistoric Europe, Proceedings of the International Colloquium, Riva del Garda 11-16 may 1998, Trento, Ufficio beni archeologici, vol. 1, p. 229-275.

Guilaine J., GUTHERZ X.

1990: «Jean Arnal: un constructeur d'hypothèses", in GuIlaine J., GUTHERZ X. (DIR.), Autour de Jean Arnal: recherches sur les premières communautés paysannes en Méditerranée occidentale, Montpellier, Laboratoire de paléobotanique, p. 11-35.

GuILAINE J., ROUdIL J.-L.

1976: "Les civilisations néolithiques en Languedoc», in GUILAINE J. (DIR.), $\mathrm{La}$ 
Préhistoire française -II- Les Civilisations néolithiques et protohistoriques de la France, Paris, CNRS Éditions, p. 267-278.

\section{Guilaine J., Tusa S., Veneroso P.}

2009: La Sicile et l'Europe campaniforme: la collection Veneroso à Sciacca, Toulouse, Archives d'écologie préhistorique, $217 \mathrm{p}$.

\section{GUILAINE J., VAQUER J.}

1976: «Début de la métallurgie dans le midi de la France et en Italie du Nord", in MÜLleR-KARPE A. (DIR.), Les Débuts de la métallurgie, colloque XXIII, Actes du IX congrès de l'UISPP, Nice, 1976, Nice, éd. de l'UISPP, prétirages, p. 46-79.

\section{GUTHERZ X.}

1975: La Culture de Fontbouisse: recherches sur le Chalcolithique en Languedoc oriental, Caveirac, ARALO (coll. Cahiers de l'ARALO, 2), $120 \mathrm{p}$.

1980a: «Les productions céramiques de l'Avencas (Brissac, Aude) dans leur contexte régional du Néolithique récent", in GUILAINE J. (DIR.), Le Groupe de Véraza et la fin des temps néolithiques dans le sud de la France et la Catalogne, Actes du colloque de Narbonne, 3-4 juin 1977, Paris, CNRS Éditions, p. 192-199.

1980b: «Le groupe de Ferrières", in GUILAINE J. (DIR.), Le Groupe de Véraza et la fin des temps néolithiques dans le sud de la France et la Catalogne, Actes du colloque de Narbonne, 3-4 juin 1977, Paris, CNRS Éditions, p. 217-222.

1982: «Une culture chalcolithique du sud-est de la France, le groupe de Fontbouisse: état actuel des connaissances", in BEST J.G.P., DE WRIES N. M. W. (DIR.), Interaction and Acculturation in the Mediterranean, Amsterdam, B. R. Grüner, p. 7-18.

1984: Les Cultures du Néolithique récent et final en Languedoc oriental, Thèse de Doctorat, Université Aix-Marseille-I, Aix-enProvence, $347 \mathrm{p}$.

1988: «Le Campaniforme du Languedoc oriental: état de la question", in BEECHING A. (DIR.) avec la collab. de LINOSSIER M., CORDIER F., DRUON A.-M., Le Campaniforme, Actes des rencontres néolithiques de Rhône-Alpes, Lyon, 11 juin 1988, Lyon (coll. ARENERA, 5), p. 64-77.

1990: «Ferrières et Fontbouisse: histoire et devenir de deux concepts», in GUIlaine J., GUTHERZ X. (DIR.), Autour de Jean Arnal: recherches sur les premières communautés paysannes en Méditerranée occidentale, Montpellier, Laboratoire de paléobotanique, p. 233-249.
1990-1991: «Acquis récents et perspectives de la recherche sur le Chalcolithique en Languedoc méditerranéen", in AMBERT P. (DIR.), Le Chalcolithique en Languedoc: ses relations extrarégionales, hommage au $D^{r}$ Jean Arnal, Actes du colloque international, Saint-Mathieu-deTréviers, Hérault, 20-22 sept. 1990, Lattes, Fédération archéologique de l'Hérault (coll. Archéologie en Languedoc, 15), p. 211-217.

1995: «Quelques réflexions sur l'origine et la chronologie du Bronze ancien dans le sud-est de la France ", in CHENORKIAN R. (DIR.), L'Homme méditerranéen: mélanges offerts à Gabriel Camps, Aix-en-Provence, Publications de l'université de Provence, p. $375-401$

\section{GUTHERZ X., JALLOT L.}

1995: «Le Néolithique final du Languedoc méditerranéen", in VORUZ J.-L. (DIR.), Chronologies néolithiques: de 6000 à 2000 avant notre ère dans le Bassin rhodanien, Actes du colloque d'Ambérieu-en-Bugey, 19-20 sept. 1992, XI rencontre sur le Néolithique de la région Rhône-Alpes, Ambérieu-en-Bugey, éd. de la SPR (coll. Documents du département d'anthropologie et d'écologie de l'université de Genève, 20), p. 231-263.

1999: «Approche géoculturelle des pays fontbuxiens", in VAQUER J. (DIR.), Le Néolithique du Nord-Ouest méditerranéen, Actes du XXIVe congrès préhistorique de France, Carcassonne, 26-30 sept. 1994, Paris, SPF, p. 161-174.

\section{GUTHERZ X., JALLOT L. avec la collab. de} BORDREUIL M.

2005: "Âge du cuivre et changements sociaux en Languedoc méditerranéen", in Ambert P., VAQUer J. (DIR.), La Première métallurgie en France et dans les pays limitrophes, Actes du colloque international de Carcassonne, 28-30 sept. 2002, Paris, SPF (coll. Mémoires de la SPF, 37), p. 119-130.

\section{HELMER D.}

1979: Recherches sur l'économie et l'origine des animaux domestiques d'après l'étude des mammifères post-paléolithiques (du Mésolithique à l'âge du Bronze) en Provence, Thèse de Doctorat, Montpellier, Université Montpellier-II, $167 \mathrm{p}$.

1986: «Le gisement de plein air chalcolithique de la Plaine-des-Blancs à Courthézon, Vaucluse: étude de la faune», $B S P F, 83,11-12$, p. 484-485.

1992: La Domestication des animaux par les hommes préhistoriques, Paris/Milan, Masson, $184 \mathrm{p}$.

\section{HONEGGER M.}

2001: L'Industrie lithique taillée du Néolithique moyen et final en Suisse, Valbonne/Paris, CNRS Éditions (coll. Monographies du CRA, 24), $353 \mathrm{p}$

2006: «Grandes lames et poignards dans le Néolithique final du nord des Alpes ", in VAQUER J., BRIOIS F. (DIR.), La Fin de l'âge de Pierre en Europe du Sud, Actes de la table ronde de l'EHESS, Carcassonne, 5-6 sept. 2003, Toulouse, éd. des Archives d'écologie préhistorique, p. 43-56.

\section{JACOB J.-P., D'ANNA A., DENANTE S.,} GUILCHER A., PAGNI M.

1987-1988: "Provence-Alpes-Côte d'Azur», Gallia Informations, Préhistoire et Histoire, 2, p. 185-343.

1990: "Provence-Alpes-Côte d'Azur», Gallia Informations, Préhistoire et Histoire, 1/2, p. 81-315.

\section{JACOBIESKI G.}

1988: Les Grottes des Meules, commune de Rochegude (Gard), Mémoire de Maîtrise, Université Paris-I, 88 p.

\section{JALLOT L.}

1988: «La station néolithique de Campguivard (Collias, Gard) : éléments pour l'étude de la céramique du groupe de Ferrières dans l'Uzège ", Archéologie en Languedoc, 4, p. 73-82.

2003: «Les Vautes dans le contexte régional du Néolithique final chalcolithique: le groupe de Ferrières dans l'Hérault et la question du "style des Vautes" ", in GUILAINe J., Escallon G. (DIR.), Les Vautes (Saint-Gély-du-Fesc, Hérault) et la fin du Néolithique en Languedoc oriental, Toulouse, EHESS/Inrap (coll. Recherches en archéologie préventive, 2), p. 235-274.

\section{JALLOT L., D'ANNA A.}

1990: "Stèles anthropomorphes et statues menhirs: état de la question et approche méthodologique nouvelle», in GUILAINE J., GUTHERZ X. (DIR.), Autour de Jean Arnal: recherches sur les premières communautés paysannes en Méditerranée occidentale, Montpellier, Laboratoire de paléobotanique, p. 359-383.

\section{JEANJEAN A.}

1884: "L'âge du Cuivre dans les Cévennes», Mémoires de l'Académie du Gard, Nîmes, p. 491-506.

\section{LAZARD N.}

2001: «Étude comparative du matériel lithique poli des sites couronniens», in 
LEMERCIER O. (DIR.), Le Couronnien en Basse-Provence occidentale: état des connaissances et nouvelles perspectives de recherches, Rapport d'activité 2001, Aix-en-Provence, ESEP/SRA Provence-Alpes-Côte d'Azur, p. 86-99.

LÉA V.

2004: Les Industries lithiques du Chasséen en Languedoc oriental: caractérisation par l'analyse technologique, Oxford, Archaeopress (coll. BAR International Series, 1232), $215 \mathrm{p}$

\section{LEMERGIER O.}

2001 : «La chronologie du Couronnien: données archéologiques et datations isotopiques", in LEMERCIER O. (DIR.), Le Couronnien en Basse-Provence occidentale: état des connaissances et nouvelles perspectives de recherches, Rapport d'activité 2001, Aixen-Provence, ESEP/SRA Provence-AlpesCôte d'Azur, p. 185-192.

2003: «La périodisation du Campaniforme dans le midi de la France: Jean Guilaine avait raison ", in GASCó J., GUTHERZ X., LABRIFFE P.-A. DE (DIR.), Temps et espaces culturels du VIe au $I^{e}$ millénaire en France du Sud, Actes des $I V^{e}$ rencontres méridionales de Préhistoire récente, Nîmes, 28-29 oct. 2000, Lattes, ADALR (coll. Monographies d'archéologie méditerranéenne, 15), p. 151-160.

2004a: Les Campaniformes dans le sudest de la France, Lattes, ARALO (coll. Monographies d'archéologie méditerranéenne, 18), 515 p.

2004b: «Explorations, implantations et diffusions: le "phénomène" campaniforme en France méditerranéenne », BSPF, 101, 2, p. 227-238.

2007: «La fin du Néolithique dans le sud-est de la France: concepts techniques, culturels et chronologiques de 1954 à 2004 ", in EVIN J. (DIR.), Un siècle de construction du discours scientifique en Préhistoire: des idées d'hier aux conceptions d'aujourd'hui, Actes du XXVI congrès préhistorique de France, congrès du centenaire de la SPF, Avignon, 21-25 sept. 2004, vol. 1, p. 485-500.

À paraître: «Révision des occupations du Néolithique moyen au Néolithique final du site des Juilléras (Mondragon, Vaucluse)", in LEMERCIER O., FURESTIER R., BAILlY M. (DIR.), IV millénaire: la transition du Néolithique moyen au Néolithique final dans le sud-est de la France et les régions voisines, Actes de la table ronde internationale, Suisse, Italie, France, Espagne, Aix-en-Provence, 11-12 mars 2005, Lattes, Publications de l'UMR 5140/
ADAL (coll. Monographies d'archéologie méditerranéenne, hors série).

\section{LEMERCIER O., BAILLY M.}

À paraître: «Les datations radiocarbone et la chronologie de la transition du Néolithique moyen au Néolithique final dans le sud-est de la France», in LEMERCIER O., FURESTIER R., BAILLY M. (DIR.), IV millénaire: la transition $d u$ Néolithique moyen au Néolithique final dans le sud-est de la France et les régions voisines, Actes de la table ronde internationale, Suisse, Italie, France, Espagne, Aix-en-Provence, 11-12 mars 2005, Lattes, Publications de l'UMR 5140/ADAL (coll. Monographies d'archéologie méditerranéenne, hors série).

\section{Lemercier O., Bailly M., Cauliez J.,} D'ANNA A., MARGARIT X., RENAULT S., VAN WILLIGEN S.

2003: «La transition du Néolithique moyen au Néolithique final, aux origines du Couronnien: approche collective des séries du "Néolithique récent" ", in LEMERCIER O. (DIR.), Le Couronnien en Basse-Provence occidentale: état des connaissances et nouvelles perspectives de recherches, Rapport d'activité 2003, Aix-en-Provence, ESEP/SRA Provence-Alpes-Côte d'Azur, p. 76-77.

Lemercier O., Blaise E., Cauliez J., Convertini F., D'AnNa A., Delaunay G., DURRENMATH G., FURESTIER R.,

Gilabert C., LAZARD N., MARGARIT X., Pellissier M., Provenzano N., RENAULT S.

2007: «Le Néolithique final couronnien en Basse-Provence occidentale, de Max Escalon de Fonton au projet collectif de recherche de l'UMR 6636 (1947-2004)", in EVINJ. (DIR.), Un siècle de construction du discours scientifique en Préhistoire: des idées d'hier aux conceptions d'aujourd'hui, Actes du XXVI congrès préhistorique de France, congrès du centenaire de la SPF, Avignon, 21-25 sept. 2004, vol. 1, p. 473-484.

Lemercier O., Blaise E., Cauliez J., FURestier R., Gallin A., Gilabert C., GUENDON J.-L., LAZARD N., Pellissier M., Piatscheck C., PROVENZANO N.

2006: "Le site Néolithique final de la Bastide blanche (Peyrolles-en-Provence, Bouches-du-Rhône): premiers résultats 2003-2004 », in FOUÉRÉ P., CHEVILLOT C., Courtaud P., Ferullo O., Leroyer C. (DIR.), Paysages et peuplement: aspects culturels et chronologiques en France méri- dionale, Actes des $V I^{e}$ rencontres méridionales de Préhistoire récente, Périgueux, 14-16 oct. 2004, Périgueux, ADRAHP (coll. Suppl. à Préhistoire du Sud-Ouest, 11), p. 473-488.

Lemercier O., Blaise E., CAUliez J., FURESTIER R., Gilabert C., LAZARD N., Pinet L., Provenzano N.

2004: «La fin des temps néolithiques", in Buisson-Catil J., GuilcheR A., Hussy C., Olive M., PAGNi M. (DIR.), Vaucluse préhistorique: le territoire, les hommes, les cultures et les sites, Le Pontet, éd. Barthélémy (coll. Archéologie), p. 201-252.

LeMERGier O., FUrestier R., MÜller A., Cauliez J., Convertini F., LAZARD N., Provenzano N. avec la collab. de Bouville C., Gilabert C., Jorda M., Khedhaier R., Loirat D., Pellissier M., VERDIN P.

2004: «Le site néolithique final de la Fare (Forcalquier, Alpes-de-Haute-Provence) : résultats 1995-1999 et révision chronoculturelle», in DARTEVELLE H. (DIR.), Auvergne et Midi, Actes des $V^{e}$ rencontres méridionales de Préhistoire récente, ClermontFerrand, 8-9 nov. 2002, Cressensac, Préhistoire du Sud-Ouest (coll. Suppl. à Préhistoire du Sud-Ouest, 9), p. 445-455.

Lemercier O., Gilabert C.

2009: De la maison au village: l'habitat néolithique dans le sud de la France et le nord-ouest méditerranéen, Actes de la table ronde de Marseille, 23-24 mai 2003, Paris, SPF (coll. Mémoires de la SPF, 48), p. 255-266.

\section{LEMERCIER O., Müller A.}

1999: «Le site Néolithique final chalcolithique de la Fare (Forcalquier, Alpesde-Haute-Provence): premiers résultats 1991-1993", in BEECHING A., VITAL J. (DIR.), Préhistoire de l'espace habité en France du Sud: actualités de la recherche, Actes des premières rencontres méridionales de Préhistoire récente, Valence-sur-Rhône, 3-4 juin 1994, Valence-sur-Rhône, CAP (coll. Travaux du CAP, 1), p. 177-183.

\section{LEMONNIER P.}

1986: «The Study of Material Culture today: toward an Anthropology of Technical Systems", Journal of Anthropological Archaeology, 5, 1, p. 147-186.

1990 : Guerres et festins: paix, échanges et compétitions dans les Highlands de Nouvelle-Guinée, Paris, éd. de la MSH, 192 p. 
LICHARDUS J., LICHARDUS-ITTEN M.

1985: «Diffusion de la civilisation néolithique en Europe et évolution historico-culturelle", in LICHARDUS J., LICHARDUSITten M. (DIR.), La Protohistoire de l'Europe: le Néolithique et le Chalcolithique, Paris, PUF (coll. Nouvelle Clio, 1), p. 207515.

\section{LIVINGSTONE-SMITH A., BOSQUET D.,} MARTINEAU R.

2005: Pottery Manufacturing Processes: Reconstitution and Interpretation, Acts of the XIVth UISPP Congress, University of Liège, Belgium, 2-8 sept. 2001, Colloque/Symposium 2.1, Cambridge, Archaeopress (coll. BAR International Series, 1349), 228 p.

\section{LOMBARD D.}

1999: «Aperçu sur le Néolithique des Hautes-Alpes », in BEECHING A. (DIR.), Circulations et identités culturelles alpines à la fin de la Préhistoire: matériaux pour une étude, Valence, CAP (coll. Travaux du CAP, 2), p. 481-500.

\section{LOUIS M.}

1948: Préhistoire du Languedoc méditerranéen et du Roussillon, Nîmes, Bruguier (coll. Cahiers d'histoire et d'archéologie), 204 p.

\section{MALLET N., RICHARD G., GENTY P., DELCOURT-VLAEMINCK M.}

2000: «La diffusion des silex du GrandPressigny au Néolithique final: état actuel de l'inventaire", Bulletin des amis du musée de Préhistoire du Grand-Pressigny, 51. p. 27-31.

\section{MARGARIT X.}

2002: "La céramique des fouilles anciennes du site de Martigues Ponteau-Gare (collection Cazenave): contribution à la chronotypologie du Néolithique final couronnien ", in LEMERCIER O. (DIR.), Le Couronnien en Basse-Provence occidentale: état des connaissances et nouvelles perspectives de recherches, Rapport d'activité 2002, Aixen-Provence, ESEP/SRA Provence-AlpesCôte d'Azur, p. 21-80.

À paraître: "Réflexions sur les caractères de la céramique du Néolithique récent méridional à travers la comparaison de deux sites rhodaniens, les Ribauds et le Duc (Mondragon, Vaucluse)», in LEMERCIER O., FURESTIER R., BAILly M. (DIR.), IV millénaire: la transition $d u$ Néolithique moyen au Néolithique final dans le sud-est de la France et les régions voisines, Actes de la table ronde internationale, Suisse, Italie, France, Espagne, Aix-en-Provence,
11-12 mars 2005, Lattes, Publications de l'UMR 5140/ADAL (coll. Monographies d'archéologie méditerranéenne, hors série).

MARGARIT X., SAINTOT S.

2002: «Le site néolithique final du Pâtis 2 à Montboucher-sur-Jabron (Drôme) ", in GUTHERZ X. (DIR), Archéologie du TGV Méditerranée, fiches de synthèse -1- La Préhistoire, Lattes, ARALO (coll. Monographies d'archéologie méditerranéenne, 8), fiche 8, p. 95-102.

\section{MARTINEAU R.}

2000: Poterie, techniques et sociétés: études analytiques et expérimentales à Chalain et à Clairvaux (Jura), entre 3200 et 2900 av. J.-C., Thèse de Doctorat, Université de Franche-Comté, 268 p.

2006: «Identification expérimentale des techniques de façonnage des poteries archéologiques", in BARAY L. (DIR.), Artisanats, sociétés et civilisations, hommage à Jean-Paul Thévenot, Actes du colloque organisé par l'UMR 5594, Dijon et le Centre de recherche et d'étude du patrimoine, Sens, 2-3 avril 2003, Dijon (coll. Suppl. à la Revue archéologique de l'Est, 24), p. 251258.

\section{Martineau R., Pétrequin P.}

2000: «La cuisson des poteries néolithiques de Chalain (Jura): approche expérimentale et analyse archéologique», in PÉTREQUIN P., FluZIN P., THIRIOT J. ET AL. (DIR.), Arts du feu et productions artisanales, Actes des $X X^{e}$ rencontres internationales d'archéologie et d'histoire d'Antibes, 21-23 oct. 1999, Juan-les-Pins, APDCA, p. $337-358$.

\section{MARTINEAU R., WALTER-SIMONNET A.-V.,} GrobÉTY B., BUATIER M.

2007: «Clay Resources and Technical Choices for Neolithic Pottery (Chalain, Jura, France): Chemical, Mineralogical and Grain-size Analyses", Archaeometry, 49, p. 23-52.

\section{MAUSS M.}

1973: «Essai sur le don: forme et raison de l'échange dans les sociétés archaïques", in MAUSS M. (DIR.), Sociologie et anthropologie, Paris, PUF (coll. Sociologie Aujourd'hui), p. 145-179.

\section{Meillassoux C.}

1977: Terrains et théories, Paris, éd. Anthropos, $344 \mathrm{p}$.

\section{MEZZENA F.}

1998: Dieux de pierre: la grande statuaire anthropomorphe en Europe au III millénaire av. J.-C., Milan, Skira, 222 p.

\section{MIARI M.}

1998: «The Rinaldone facies: the funeral ritual", in DE MARINIS R.-C., BIETTI Sestieri A.-M., Peroni R., Peretto C. (DIR.), The Copper Age in the Near East and Europe, The Bronze Age in Europe and the Mediterranean, The Iron Age in Europe, Acts of the XIIIth UISPP Congress, Forli, 8-14 sept. 1996, Section 4, Forlì, Abaco, p. 61-70.

\section{MOINAT P., STÖCKLI W-E.}

1995: «Croyances et rites funéraires: la Suisse du Paléolithique à l'aube du Moyen Âge », in STÖCKLI W. E., NIfFEler U., GROSSKLEE E. (DIR.), Néolithique, Bâle, Société suisse de Préhistoire et d'archéologie (coll. La Suisse du Paléolithique à l'aube du Moyen Âge, 2), p. 231-258.

\section{MONNET G., BAZILE F., GEORJON C.} Di PIETRO-SIRVEN R.

2002: "Rochefort-du-Gard, la Grange des Merveilles I: un habitat du Néolithique récent", in GUTHERZ X. (DIR.), Archéologie du TGV Méditerranée, fiches de synthèse -1- La Préhistoire, Lattes, ARALO (coll. Monographies d'archéologie méditerranéenne, 8), fiche 27, p. 265-274.

\section{MONTJARDIN R.}

1965: «Le peuplement préhistorique de Chauzon", Cahiers rhodaniens, 12, p. $24-40$.

1966: «Schéma des formes et décors du Chalcolithique de la basse Ardèche», Ogam, 18, p. 17-22.

1988-1989: «La céramique de Ferrières», Thau: histoire et archéologie, 4, p. 1-52.

1990-1991: «Le Chalcolithique dans l'Ardèche ", in AMBERT P. (DIR.), Le Chalcolithique en Languedoc et ses relations extrarégionales, hommage au $D^{r}$ Jean Arnal, Actes du colloque international, Saint-Mathieu-deTréviers, Hérault, 20-22 sept. 1990, Lattes, Fédération archéologique de l'Hérault (coll. Archéologie en Languedoc, 15), p. 227-244.

\section{MORIN A.}

2000: «État documentaire sur le Néolithique final dans la zone préalpine: massifs et piémonts de la Chartreuse, du Vercors et du pays du Buëch, in TILlET T. (DIR.), Les Paléoalpins, hommage à Pierre Bintz, Grenoble, Université Grenoble-I (coll. Géologie alpine, 31), p. 211-229. 
2005: «Les ambiances culturelles néolithiques "haut-alpines" et leur insertion dans le contexte du Bassin rhodanien", in NICAUlT J. (DIR.), Vie, culture et sociétés dans les Alpes, Actes du colloque international d'histoire et d'archéologie sur l'Arc alpin, Gap, 28-29 sept. 2002, Gap, Louis-Jean impr., p. 29-56.

MORIN A. avec la collab. de BRESSY C., COUSSERAN S., BERNARD C.

2000: Rapport de prospection thématique néolithique dans la vallée du Buëch: bassins des torrents de la Maraize et du Déoule (La Bâtie-Montsaléon, Chabestan, Le Saix, SaintAuban-d'Oze, Esaparron, Barcillonnette), Grenoble, Université de Provence/SRA Provence-Alpes-Côte d'Azur, 66 p.

Morin A., PiCAVET R. avec la collab. de CARLES J.

2006: "Les poignards gravés découverts à Chastel-Arnaud (Drôme) ", in JOURDAINANNEQUIN C. (DIR.), Aires culturelles, aires linguistiques dans les Alpes occidentales, Actes du colloque, Grenoble, 18-19 nov. 2004, Grenoble, éd. CRHIPA (coll. Les Cahiers du CRHIPA, 8), p. 67-80.

MORIN A., PICAVET R. avec la collab. de CARLES J., BERNARD C.

2005: "Étude préliminaire sur des poignards gravés de type Remedello découverts dans les Préalpes du Sud (Chastel-Arnaud, Drôme, France) et réflexion sur leur insertion dans le Néolithique final régional», BSPF, 102, p. 345-359.

MÜller A., Crégut-Bonnoure E., Guérin C., Barthélémy A., PHILIPPE M., Helmer D., SUdRE C., LAVERGNe D., OLLIVIER V., FUTOUR O. (DIR.)

2005: Marc Deydier: 1845-1920, Périgueux, Patrilub, 239 p.

MÜLLER A., D'ANNA A. avec la collab. de BRANDI R., BRETAGNE P., MAURIN M.

1986: «Le gisement de plein air chalcolithique de la Plaine-des-Blancs à Courthézon, Vaucluse », BSPF, 83, p. 470-483.

MURET A.

1991: «Le gisement archéologique du col des Tourettes (Montmorin, Hautes-Alpes)», in BARRUOL A. (DIR.), Archéologie dans les Hautes-Alpes, Gap, Louis-Jean impr., p. 81-88.

1996: «Huit campagnes de fouilles archéologiques au col des Tourettes à Montmorin (Hautes-Alpes) », in Archéologie en Baronnies, Actes des $I^{e}$ rencontres du Gardenotes baronniard, Lachau, 22 oct. 1995,
Buis-les-Baronnies, éd. Le Garde-notes Baronniard, p. 53-63.

\section{ObERLIN C.}

2003: "Calibration des datations radiocarbone: le point sur la période $\mathrm{VI}^{\mathrm{e}}$ $\mathrm{II}^{\mathrm{e}}$ millénaire av. J.-C.», in GASCÓ J., GUTHERZ X., LABRIFFE P.-A. DE (DIR.), Temps et espaces culturels du $\mathrm{VI}^{e}$ au $\mathrm{II}^{e}$ millénaire en France du Sud, Actes des IV rencontres méridionales de Préhistoire récente, Nimes, 28-29 oct. 2000, Lattes, ADALR (coll. Monographies d'archéologie méditerranéenne, 15), p. 35-42.

\section{Pellissier M.}

2001: «La parure des sites du Collet-Redon et de Ponteau-Gare à Martigues (Bouchesdu-Rhône) dans son contexte couronnien", in LEMERCIER O. (DIR.), Le Couronnien en Basse-Provence occidentale: état des connaissances et nouvelles perspectives de recherches, Rapport d'activité 2001, Aixen-Provence, ESEP/SRA Provence-AlpesCôte d'Azur, p. 100-127.

\section{PÉTREQUIN P.}

1997a: «Variations démographiques et transferts culturels", in PÉTREQUIN P. (DIR.), Les Sites littoraux néolithiques de Clairvauxles-Lacs et de Chalain (Jura) -3- Chalain station 3, 3200-2900 av. J.-C., Paris, éd. de la MSH (coll. Archéologie et culture matérielle), p. 319-323.

1997b: «Dynamique d'expansion culturelle et croissance démographique", in PÉTRequin P. (DIR.), Les Sites littoraux néolithiques de Clairvaux-les-Lacs et de Chalain (Jura) -3- Chalain station 3, 3200-2900 av. J.-C., Paris, éd. de la MSH (coll. Archéologie et culture matérielle), p. 563-575.

2002: «Produir i bescanviar signes socials: ornaments, punyals i destrals de pedra al neolític europeu », Cota Zero, 17, p. 82-97.

\section{Pétrequin P., Bailly M., Viellet A.}

1998: «Les villages littoraux néolithiques du Jura français et les chronologies des IV ${ }^{\mathrm{e}}$ et III ${ }^{\mathrm{e}}$ millénaire av. J.-C.: le point de vue de l'archéologue et du dendrochronologue », in BARRANDON J.-N., GUIBERT P., MiCHEL V. (DIR.), Datation, Actes des $X X I^{e}$ rencontres internationales d'histoire et d'archéologie d'Antibes, 19-21 oct. 2000, Juan-les-Pins, APDCA, p. 401-431.

\section{Pétrequin P., Chastel J., Giligny F.,} PÉTReQuin A.-M., SAintot S.

1987-1988: «Réinterprétation de la civilisation Saône-Rhône: une approche des tendances culturelles du Néolithique final», Gallia Préhistoire, 30, p. 1-89.

Pétrequin P., Pétrequin A.-M. avec la collab. de CORAS J., DARTEVELLE H., MAÎTRE A., ROSSY M.

1993: Écologie d'un outil: la hache de pierre polie en Irian Jaya (Indonésie), Paris, CNRS Éditions (coll. Monographies du CRA, 12), $439 \mathrm{p}$.

\section{Pétrequin P., Pétrequin A.-M.,}

GILIGNY F., RUBY P.

1994: «Produire pour soi, la céramique de Chalain 2C au Néolithique final ", BSPF, 91, 6, p. 407-417.

\section{Peyrolles D., Arnal J.}

1954: «La poterie cannelée du type Fontbouisse », Zephyrus, 5, p. 165-185.

\section{PeYrolles D., LOUIS M.}

1946: «Stations préhistoriques des environs de Sommières", Cahiers d'histoire et d'archéologie, 1, p. 97-102.

\section{Piatscheck C.}

2004: La Fare à Forcalquier (Alpes-de-HauteProvence): technologie et typologie de l'outillage lithique taillé, Mémoire de Maîtrise, Université Aix-Marseille-I, Aixen-Provence, $168 \mathrm{p}$.

2005: Chaînes opératoires et évolution culturelle dans le Néolithique final provençal: étude technologique de l'industrie lithique taillée de l'occupation de la Fare (Forcalquier, Alpes-de-Haute-Provence), Mémoire de Master 2, Université Aix-Marseille-I, Aixen-Provence, 143 p.

\section{RAMSEYER D.}

1987: Delley/Portalban II: contribution à l'étude du Néolithique en Suisse occidentale, Fribourg, Éditions universitaires Fribourg (coll. Archéologie fribourgeoise, 3), $118 \mathrm{p}$.

\section{RAYMOND P.}

1897: «Deux grottes sépulcrales dans le Gard: contribution à l'âge du Cuivre dans les Cévennes", Bulletin de la Société anthropologique de Paris, IV e série, t. 8, p. $65-75$.

1898: «Nouvelles recherches sur l'âge du Cuivre dans les Cévennes (Époque durfortienne) », Bulletin de la Société d'anthropologie de Paris, IV érie, t. 9, p. 50-56.

\section{RENAULT S.}

1990: Le Gisement de plein air des Martins, Roussillon, Vaucluse: étude de l'indus- 
trie lithique, Mémoire de DEA, Aix-enProvence, Université de Provence, 59 p.

1998: «Économie de la matière première: l'exemple de la production au Néolithique final, en Provence, des grandes lames en silex zoné oligocène du bassin de Forcalquier (Alpes-de-HauteProvence) », in D'ANNA A., BINDER D. (DIR.), Production et identité culturelle: actualité de la recherche, Actes des $I I^{e}$ rencontres méridionales de Préhistoire récente, Arles, 8-9 nov. 1996, Antibes, APDCA, p. $145-161$.

2006: «La production des grandes lames au Néolithique final en Provence: matériaux exploités, multiplicité des productions, aspects technologiques et chronoculturels ", in VAQUER J., BRIOIS F. (DIR.), La Fin de l'âge de Pierre en Europe du Sud, Actes de la table ronde de l'EHESS, Carcassonne, 5-6 sept. 2003, Toulouse, éd. des Archives d'écologie préhistorique, p. 139-164.

\section{Renfrew C., Cherry J. F.}

1986: «Introduction: Peer Polity Interaction and Socio-political Change», in Renfrew C., Cherry J.-F. (DIR.), Peer Polity Interaction and Socio-Political Change, Cambridge, Cambridge University Press (coll. New Directions in Archaeology), p. 1-18.

\section{RODOT M.-A., MARTINEAU R.,}

\section{Bonvalot J., GaleTti G.}

2005: "Nature et provenance des matériaux céramiques Horgen (3179-3118 av. J.-C.) de Montilier/Platsbünden », Cahiers d'archéologie fribourgeoise, 7, p. 90-113.

\section{ROGER J.-M.}

1988: «Le Campaniforme en Vaunage: vestiges diffus et structures évidentes", in BEECHING A. (DIR.) avec la collab. de LiNOSSIER M., CORDIER F., DRUON A.-M., Le Campaniforme, Actes des rencontres néolithiques de Rhône-Alpes, Lyon, 11 juin 1988, Lyon (coll. ARENERA, 5), p. 80-109.

1995: «Du Chalcolithique au Bronze ancien dans le Gard: rupture ou continuité ? Une question de faciès ", in VORUZ J.-L. (DIR.), Chronologies néolithiques: de 6000 à 2000 avant notre ère dans le Bassin rhodanien, Actes du colloque d'Ambérieu-en-Bugey, 19-20 sept. 1992, XI rencontre sur le Néolithique de la région Rhône-Alpes, Ambérieu-en-Bugey, SPR (coll. Documents du département d'Anthropologie et d'Écologie de l'université de Genève, 20), p. 287-306.

\section{Rossi M., GATtiglia A.}

1998: «La Préhistoire récente d'une région de haute et moyenne montagne: le
Briançonnais (Hautes-Alpes) », in D'ANNA A., BINDER D. (DIR.), Production et identité culturelle: actualité de la recherche, Actes des $I I^{e}$ rencontres méridionales de Préhistoire récente, Arles, 8-9 nov. 1996, Antibes, APDCA, p. 447-461.

2005: «Les poignards de Remedello hors d'Italie: révision de données", in AMbert P., VAOUER J. (DIR.), La Première métallurgie en France et dans les pays limitrophes, Actes du colloque international de Carcassonne, 28-30 sept. 2002, Paris, SPF (coll. Mémoires de la SPF, 37), p. 265271

\section{ROUDIL J.-L.}

1972: L’Âge du Bronze en Languedoc oriental, Paris, Klincksieck (coll. Mémoires de la SPF, 10), $303 \mathrm{p}$.

\section{SAHLINS M.}

1976: Âge de pierre, âge d'abondance: l'économie des sociétés primitives, Paris, Gallimard (coll. Bibliothèque Sciences humaines), $412 \mathrm{p}$.

\section{SAINT-VENANT J. DE}

1908: «Les premiers âges des Métaux dans le Gard", in Actes du IV congrès préhistorique de France, Chambéry, 23-30 août 1908, Chambéry, Imprimerie générale savoisienne, p. 628-638.

\section{SALANOVA L.}

2000: La Question du Campaniforme en France et dans les îles anglo-normandes: productions, chronologie et rôle d'un standard céramique, Paris, Comité des travaux historiques et scientifiques/SPF, $392 \mathrm{p}$.

2004: «Le rôle de la façade atlantique dans la genèse du Campaniforme en Europe ", BSPF, 101, 2, p. 223-226.

\section{SANGMEISTER E.}

2005: «Les débuts de la métallurgie dans le sud-ouest de l'Europe», in AMBERT P., VAQUER J. (DIR.), La Première métallurgie en France et dans les pays limitrophes, Actes du colloque international de Carcassonne, 28-30 sept. 2002, Paris, SPF (coll. Mémoires de la SPF, 37), p. 19-25.

\section{SAUlieU G. DE}

2004: Art rupestre et statues menhirs dans les Alpes: des pierres et des pouvoirs 30002000 av. J.-C., Paris, éd. Errance (coll. Hespérides), $191 \mathrm{p}$.

SAUZAde G.

1983: Les Sépultures du Vaucluse, du Néolithique à l'âge du Bronze, Paris, Laboratoire de paléontologie humaine et de Préhistoire/
Institut de paléontologie humaine (coll. Études quaternaires, 6), $251 \mathrm{p}$.

1988: «Jouques, Saint-Bachi», Notes dinformation et de liaison de la Direction des Antiquités de Provence-Alpes-Côte d'Azur, 5 , p. 83-86.

1990a: «Les dolmens de la Provence occidentale et la place des tombes de Fontvieille dans l'architecture mégalithique méridionale", in GUILAINe J., GUTHERZ X. (DIR.), Autour de Jean Arnal: recherches sur les premières communautés paysannes en Méditerranée occidentale, Montpellier, Laboratoire de paléobotanique, p. 305334.

1990b: «Ménerbes, Falabrègue, grotte Goulard: habitat du Néolithique final», Notes d'information et de liaison de la Direction des Antiquités de Provence-AlpesCôte d'Azur, 7, p. 184-186.

Sauzade G., Courtin J., Chambert A.

1990: «Un nouveau faciès du Néolithique final provençal: le groupe de Fraischamp, l'habitat de la Clairière à la Roque-surPernes, Vaucluse», Gallia Préhistoire, 32, p. 151-178.

\section{SÉNÉPART I.}

1995: "Chronologie des industries osseuses néolithiques dans le sud-est de la France», in VORUZ J.-L. (DIR.), Chronologies néolithiques: de 6000 à 2000 avant notre ère dans le Bassin rhodanien, Actes du colloque d'Ambérieu-en-Bugey, 19-20 sept. 1992, $X I^{e}$ rencontre sur le Néolithique de la région Rhône-Alpes, Ambérieu-en-Bugey, SPR (coll. Documents du département d'Anthropologie et d'Écologie de l'université de Genève, 20), p. 205-216.

\section{SHENNAN S.}

1990: «Introduction: archaeological approaches to cultural identity ", in SHENNAN S. (DIR.), Archaeological approaches to cultural identity, London, Unwin Hyman, p. 1-32.

\section{STEINIGER D.}

2005: «L'Énéolithique en Italie», in AMbert P., VAQUER J. (DIR.), La Première métallurgie en France et dans les pays limitrophes, Actes du colloque international de Carcassonne, 28-30 sept. 2002, Paris, SPF (coll. Mémoires de la SPF, 37), p. 287-301.

\section{STRAHM C.}

1982: «Zu den Begriffen Chalkolithikum und Metallikum ", in Il Passaggio dal Neolitico all'età del Bronzo nell'Europa centrale e nella regione alpina: problemi cronologici e terminologici, Atti del X. Simposio internazionale 
sulla fine del Neolitico e gli inizi dell'età del Bronzo in Europa, Lazise-Verona 8-12 apr. 1980, Vérone, Museo civico di Storia naturale, p. 13-26.

1996: «Le concept Bronze ancien», in MORDANT C., GAIFFE O. (DIR.), Cultures et sociétés du Bronze ancien en Europe, Actes du $117^{e}$ congrès national des sociétés savantes, Clermont-Ferrand, 1992, Paris, éd. du CTHS (coll. Actes des congrès nationaux des sociétés historiques et scientifiques), p. 667-674.

2004: «Le phénomène campaniforme et les composantes autochtones non campaniformes », BSPF, 101, 2, p. 201-206.

2005: "L'introduction et la diffusion de la métallurgie en France", in AMBERT P., VAQUER J. (DIR.), La Première métallurgie en France et dans les pays limitrophes, Actes $d u$ colloque international de Carcassonne, 28-30 sept. 2002, Paris, SPF (coll. Mémoires de la SPF, 37), p. 27-36.

Thévenot J.-P., Strahm C., BeEching A., Bill J., BocQuet A., Gallay A. PÉTREQUiN P., SCHIFFERdECKER F.

1976: «La civilisation Saône-Rhône», Revue archéologique de l'Est et du Centre-Est, 27, 3-4, p. 332-420.

\section{Thirault E.}

2004: Échanges néolithiques: les haches alpines, Montagnac, éd. Monique Mergoil (coll. Préhistoire, 10), 468 p.

\section{TREMBLAY R.}

1999: «Culture et ethnicité en archéologie: les aléas de l'identité conjuguée au passé", Recherches amérindiennes au Québec, 29, 1 p. 3-8.

\section{VAN BERG P.-L.}

1983: «Une nouvelle approche du décor céramique au Néolithique ancien et son application à la céramique du Limbourg ", in DE LAET S.-J. (DIR.), Progrès récents dans l'étude du Néolithique ancien, Colloque international, Gand, 21-22 mai 1982, Bruges, De Tempel (coll. Dissertationes archaeologicae Gandenses, 21), p. 103-112.

\section{VAN WILLIGEN S.}

À paraître: «La stratigraphie du Mourre de la Barque (Jouques, Bouches-du-Rhône) et la fin du Néolithique moyen en Provence occidentale », in LEMERCIER O., FURESTIER R., BAILlY M. (DIR.), $I V^{e}$ millénaire: la transition du Néolithique moyen au Néolithique final dans le sud-est de la France et les régions voisines, Actes de la table ronde internationale, Suisse, Italie, France, Espagne, Aix-en-Provence, 11-12 mars 2005,
Lattes, Publications de l'UMR 5140/ ADAL (coll. Monographies d'archéologie méditerranéenne, hors série).

\section{VAQUER J.}

1990: Le Néolithique en Languedoc occidental, Paris, CNRS Éditions, $400 \mathrm{p}$

1998: «Le Midi méditerranéen de la France», in GUILAINE J. (DIR.), Atlas du Néolithique européen : l'Europe occidentale, Université de Liège (coll. Études et recherches archéologiques de l'université de Liège, 46), vol. 2, p. 413-500.

2007: «Les importations d'outils sur grandes lames ou sur plaquettes de silex du Néolithique récent au Chalcolithique dans le domaine nord-pyrénéen: des réseaux de concurrence ?", in BESSE $\mathrm{M}$ (DIR.), Sociétés néolithiques: des faits archéologiques aux fonctionnements socioéconomiques, Actes du XXVII colloque interrégional sur le Néolithique, Neuchâtel, 1-2 oct. 2005, Lausanne (coll. Cahiers d'archéologie romande, 108), p. 69-81.

VAQUER J., REMICOURT M., VERGÉLY H.

2006: «Les poignards métalliques et lithiques du Chalcolithique pré-campaniforme des petits et grands Causses dans le midi de la France », in GASCó J., LEYGE F., GRUAT P. (DIR.), Hommes et passé des Causses, hommage à Georges Costantini, Actes du colloque de Millau, 16-18 juin 2005, Toulouse, éd. des Archives d'écologie préhistorique, p. 155-179.

\section{VITAL J.}

1996: «État des travaux sur l'âge du Bronze ancien médio-rhodanien ", in MORdANT C., GAIFFE O. (DIR.), Cultures et sociétés du Bronze ancien en Europe, Actes du $117^{e}$ congrès national des sociétés savantes, Clermont-Ferrand, 1992, Paris, éd. du CTHS (coll. Actes des congrès nationaux des sociétés historiques et scientifiques), p. 325-338.

2002: «La séquence holocène et les occupations des âges des Métaux de Roynac-Le Serre 1 (Drôme)", in GUICHARD V., DEDET B. (DIR), Archéologie du TGV Méditerranée, fiches de synthèse -2- La Protohistoire, Lattes, ARALO (coll. Monographies d'archéologie méditerranéenne, 9, fiche 42), p. 411-426.

2004: «Ruptures et continuités du Néolithique final au Bronze ancien dans la vallée du Rhône (France) : nouveaux éléments de compréhension", in BEIER H.-J., EINICKE R. (DIR.), Varia neolitica III, Beiträge zur Ur-und frühgeschichte Mitteleuropas, Langenweissbach, Beier und Beran Velag, p. 251-277. 2005a: «Du Néolithique final au Bronze moyen dans le sud-est de la France: 22001450 av. J.-C. », Cypsela, 5, p. 11-38.

2005b: "Approche typologique des céramiques de l'âge du Bronze et perspectives: un résumé", in ROSEN J. (DIR.), Bilan critique des approches céramologiques du Néolithique à l'époque moderne, Actes de la journée d'exposés-débats, 6 déc. 2004, Dijon, UMR 5594, p. 23-31.

2006: «Les fouilles 1981-1987 dans la grotte de la Chauve-Souris à Donzère (Drôme) : visées initiales, problématiques actuelles, premières caractérisations chronoculturelles, implications pour le sud-est de la France et le domaine circum-alpin", in FOUÉré P., CHEVIllot C., Courtaud P., Ferullo O., Leroyer C. (DIR.), Paysages et peuplement: aspects culturels et chronologiques en France méridionale, Actes des VI rencontres méridionales de Préhistoire récente, Périgueux, 14-16 oct. 2004, Périgueux, ADRAHP (coll. Suppl. à Préhistoire du Sud-Ouest, 11), p. 257-292.

2008: «La séquence néolithique final-bronze ancien dans l'axe rhodanien: enseignements chronométriques et perspectives culturelles », BSPF, 105, 3, p. 539-554.

À paraître: «Les séquences céramiques des Balmes de Sollières-Sardières (Savoie) et de la grotte de la Chauve-Souris à Donzère (Drôme): implications sur le Néolithique final transalpin, le phasage et le concept Remedello", in BeEching A., Billaud Y., LE BOTHelly A., MARTiN G., Thirault E., Vital J., Cordier F., Jallet F., SAINTOT S., (DIR.), Économie et société à la fin de la Préhistoire, Actes des VII Rencontres méridionales de Préhistoire récente, 3-4 nov. 2006, Lyon.

\section{VORUZ J.-L.}

1984: Outillage osseux et dynamisme industriel dans le Néolithique jurassien, Lausanne, Bibliothèque historique vaudoise et Université de Genève (coll. Cahiers d'archéologie romande, 29), $533 \mathrm{p}$.

1991: Le Néolithique suisse: bilan documentaire, Genève, Université de Genève (coll. Documents du département d'anthropologie et d'écologie, 16), 176 p.

VORUZ J.-L. (DIR.)

1995: Chronologies néolithiques: de 6000 à 2000 avant notre ère dans le Bassin rhodanien, Actes du colloque d'Ambérieu-en-Bugey, 19-20 sept. 1992, XI rencontre sur le Néolithique de la région Rhône-Alpes, Ambérieu-en-Bugey, SPR (coll. Documents du département d'Anthropologie et d'Écologie de l'université de Genève, 20), 420 p. 
VORUZ J.-L., NICOD P.-Y., CEUNINCK G. DE 1995: «Les chronologies néolithiques dans le bassin rhodanien: un bilan», in VORUZ J.-L. (DIR.), Chronologies néolithiques: de 6000 à 2000 avant notre ère dans le Bassin rhodanien, Actes du colloque d'Ambérieu-en-Bugey, 19-20 sept. 1992, XI rencontre sur le Néolithique de la région Rhône-Alpes, Ambérieu-en-Bugey, SPR (coll. Documents du département
d'Anthropologie et d'Écologie de l'université de Genève, 20), p. 381-404.

WOLF G.

1990-1991: «Les relations entre les cultures du Midi et de Suisse occidentale au Néolithique récent et final», in AMBERT P. (DIR.), Le Chalcolithique en Languedoc et ses relations extrarégionales, hommage au $D^{r}$ Jean Arnal, Actes du colloque international, SaintMathieu-de-Tréviers, Hérault, 20-22 sept.
1990, Lattes, Fédération archéologique de l'Hérault (coll. Archéologie en Languedoc, 15), p. 337-346.

1993: Die Seeufersiedlung Yverdon, Avenue des Sports (Kanton Waadt). Eine kulturgeschichtliche und chronologische Studie zum Endneolithikum der Westschweiz und angrenzender Gebiete, Lausanne, Bibliothèque historique vaudoise (coll. Cahiers d'archéologie romande, 59), $450 \mathrm{p}$. 